\title{
Calibration of PS09, PS10, and PS11 Trans-Alaska Pipeline System Strong-Motion Instruments, with Acceleration, Velocity, and Displacement Records of the Denali Fault Earthquake, 03 November 2002
}

By John R. Evans ${ }^{1}$, E. Gray Jensen ${ }^{1}$, Russell Sell ${ }^{1}$, Christopher D. Stephens ${ }^{1}$, Douglas J. Nyman ${ }^{2}$, Robert C. Hamilton ${ }^{3}$, and William C. Hager ${ }^{4}$

Open-File Report 2006-1028

2006

Any use of trade, firm, or product names is for descriptive purposes only and does not imply endorsement by the U.S. Government.

U.S. DEPARTMENT OF THE INTERIOR

U.S. GEOLOGICAL SURVEY

${ }^{1}$ U.S. Geological Survey, 345 Middlefield Rd, MS-977, Menlo Park CA 94025.

${ }^{2}$ D. J. Nyman \& Associates, 12337 Jones Road, Suite 232, Houston TX 77070.

${ }^{3}$ Consultant, 10226 Sugar Hill Dr, Houston TX 77042.

${ }^{4}$ Alyeska Pipeline Service Company, 615 Bidwill St, Fairbanks AK 99701. 


\section{TABLE OF CONTENTS}

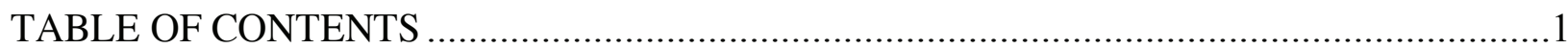

INTRODUCTION .......................................................................................................

STATION COORDINATES AND ORIENTATIONS …..............................................

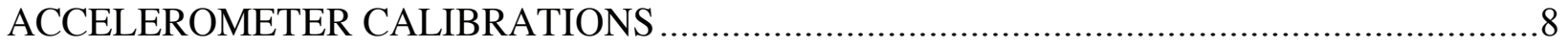

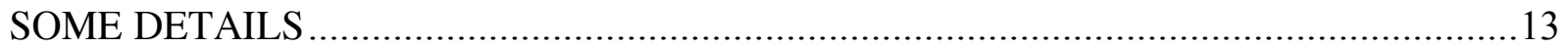

CALIBRATION OF THE REFTEK ${ }^{\mathrm{TM}}$ SEISMOGRAPH .......................................... 15

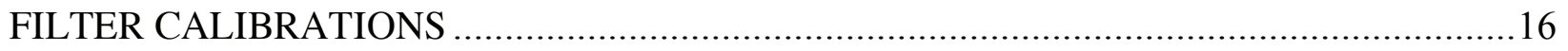

BASELINE STABILITY AND SENSOR DOUBLE INTEGRATION TESTS ......................18

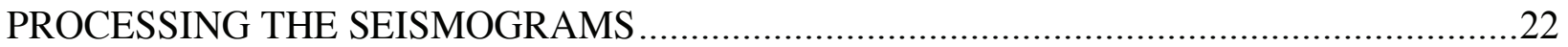

SIGNAL FLOW AND GOVERNING EQUATIONS..................................................24

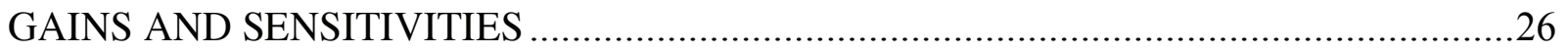

DECONVOLUTION AND DOUBLE INTEGRATION OF THE SEISMOGRAMS............27

Demeaning and Removal of Acceleration Step .................................................29

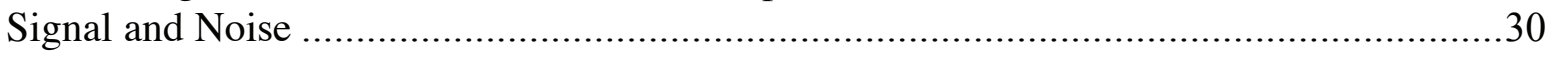

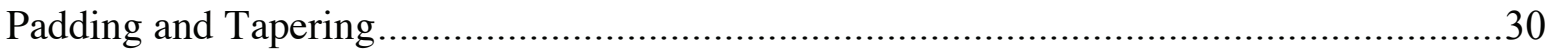

Butterworth "Error Filter" and PS10 Causal Processing ..............................................35

Acausal Processing, All Stations........................................................................ 41

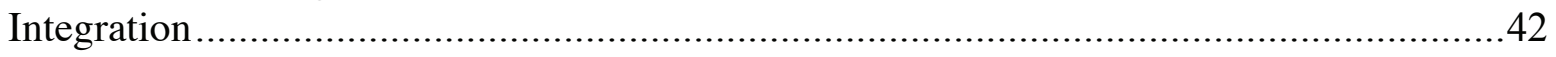

Comments on Interpreted ("Volume 2") Seismograms............................................46

SEISMOGRAMS RELEASED WITH THIS REPORT …..............................................5.

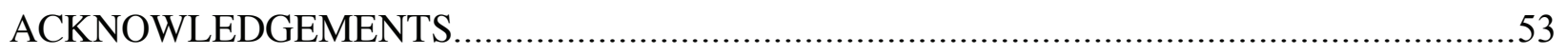

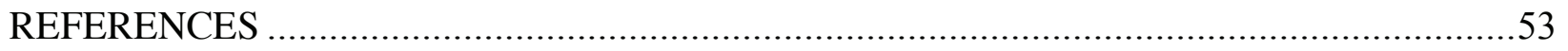




\title{
Calibration of PS09, PS10, and PS11 Trans-Alaska Pipeline System Strong-Motion Instruments, with Acceleration, Velocity, and Displacement Records of the Denali Fault Earthquake, 03 November 2002
}

\author{
J. R. Evans, E. G. Jensen, R. Sell, and C. D. Stephens, U.S. Geological Survey; \\ D. J. Nyman, D. J. Nyman \& Associates; R. C. Hamilton, Consultant; and \\ W. C Hager, Alyeska Pipeline Service Company
}

\section{INTRODUCTION}

In September, 2003, the Alyeska Pipeline Service Company (APSC) and the U.S. Geological Survey (USGS) embarked on a joint effort to extract, test, and calibrate the accelerometers, amplifiers, and bandpass filters from the earthquake monitoring systems (EMS) at Pump Stations 09, 10, and 11 of the Trans-Alaska Pipeline System (TAPS). These were the three closest strong-motion seismographs to the Denali fault when it ruptured in the $\mathrm{M}_{\mathrm{w}} 7.9$ earthquake of 03 November 2002 (22:12:41 UTC), and are shown in Figure 1. The surface rupture is only $3.0 \mathrm{~km}$ from PS10 and $55.5 \mathrm{~km}$ from PS09 but PS11 is $124.2 \mathrm{~km}$ away from a small rupture splay and $126.9 \mathrm{~km}$ from the main trace.

Here we briefly describe precision calibration results for all three instruments. Included with this report is a link to the seismograms reprocessed using these new calibrations:

- http://nsmp.wr.usgs.gov/data_sets/20021103_2212_taps.html

Calibration information in this paper applies at the time of the Denali fault earthquake (03 November 2002), but not necessarily at other times because equipment at these stations is changed by APSC personnel at irregular intervals. In particular, the equipment at PS09, PS10, and PS11 was changed by our joint crew in September, 2003, so that we could perform these calibrations. The equipment stayed the same from at least the time of the earthquake until that retrieval, and these calibrations apply for that interval. 


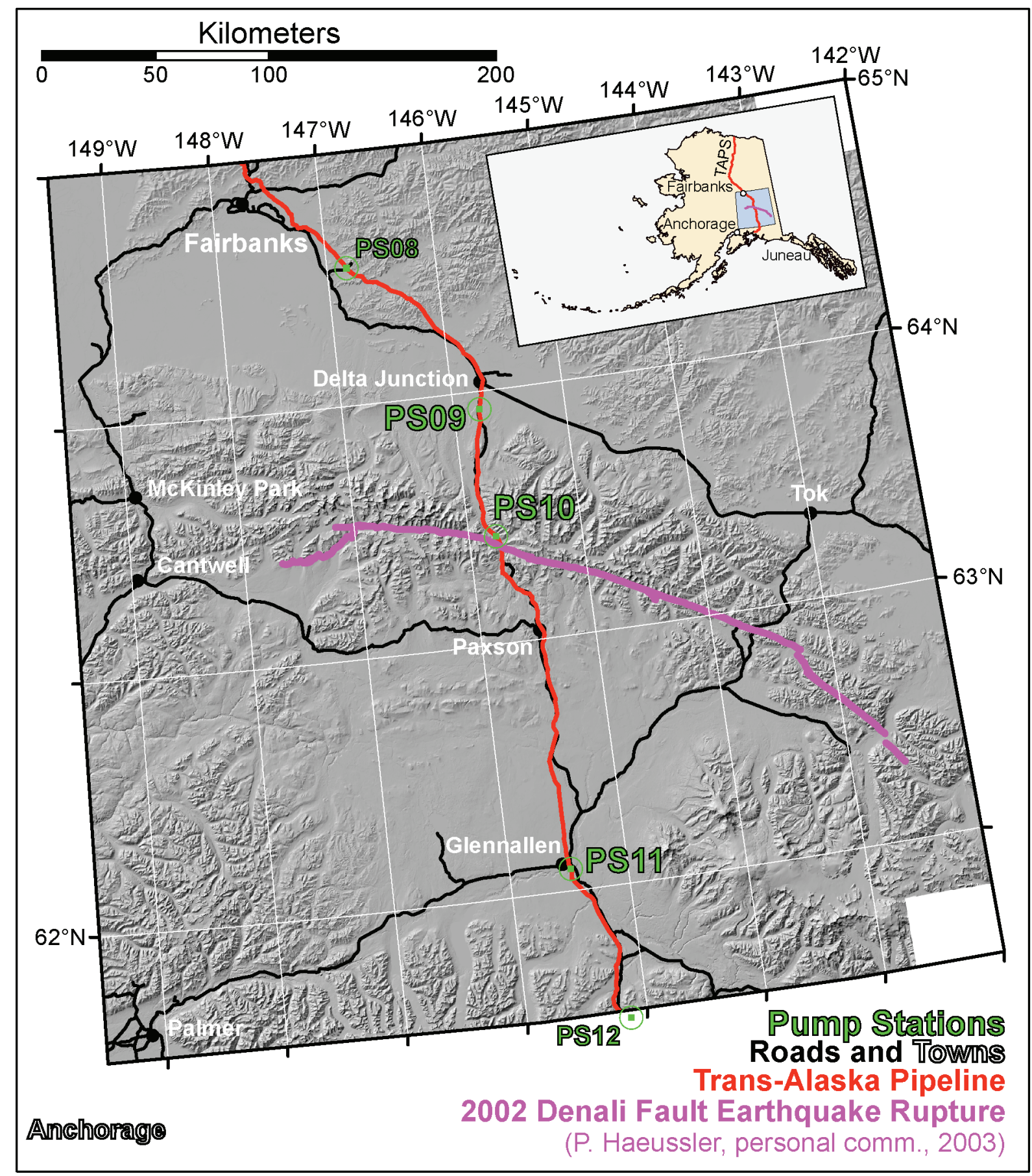

Figure 1. A map of the Denali fault earthquake rupture trace (magenta), the Trans-Alaska Pipeline System (TAPS; red), Pump Stations 8 through 12 (green; of these only 09 and 12 are actively pumping but all have instruments which recorded the 03 November 2002 earthquake), and topography, towns, and roads. Inset shows map's location within Alaska, TAPS, rupture trace, and major cities. 


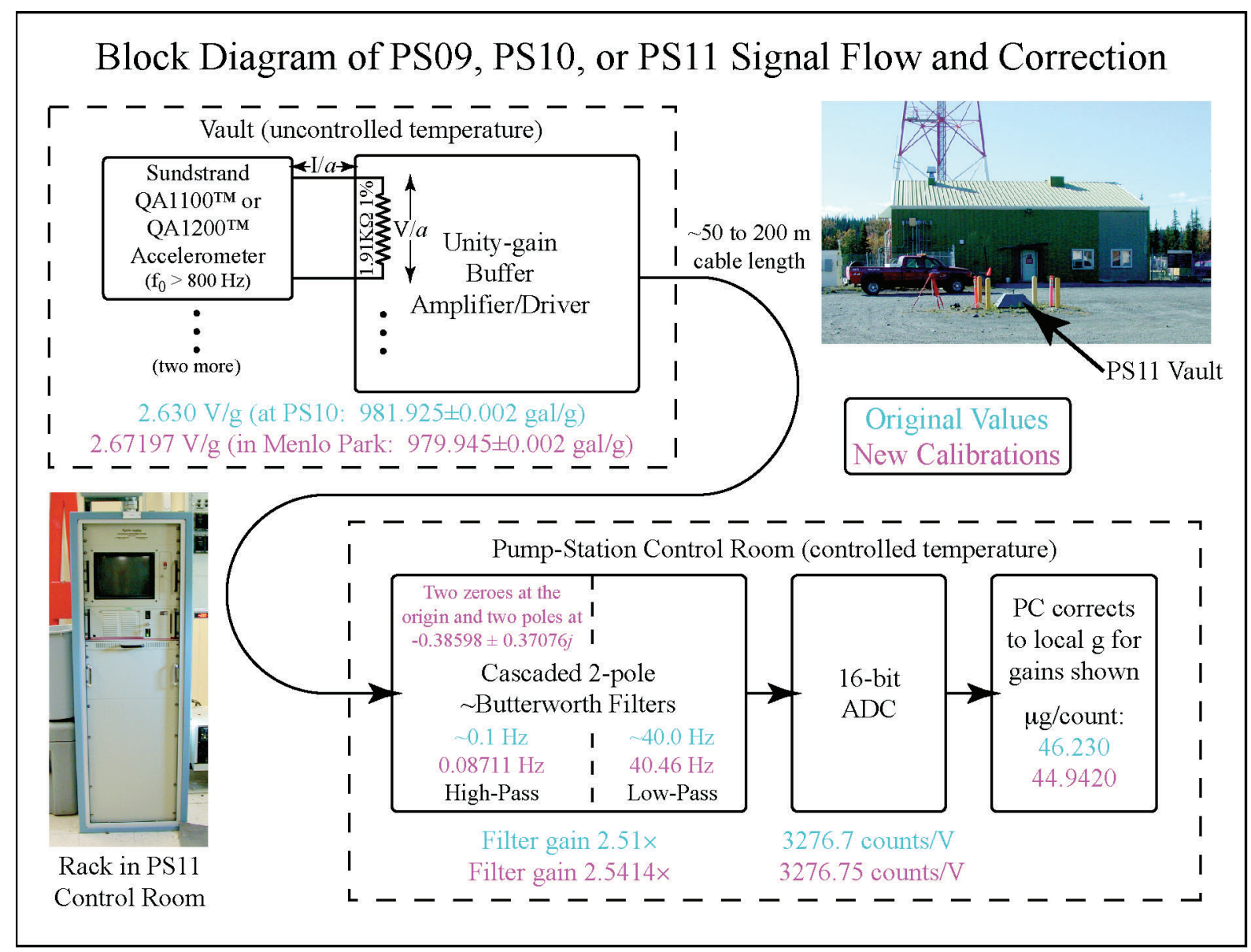

Figure 2. Diagram of signal flow from accelerometers through the recording system in the form it stood on 03 November 2002 during the $\mathrm{M}_{\mathrm{W}} 7.9$ Denali fault earthquake. (We measured only buffered "V/a" values at the output of the amplifier, never "I/a".) Inset photographs are of PS11 the following September. Equipment at the other sites is similar, but distances from the vault to the recording building are significantly larger. As an example, gains for PS10, "North", are shown in cyan for the original values and magenta for our new calibration results. No filter deconvolution was applied to previously released seismograms; high-pass filters are deconvolved from the interpreted ("Volume 2") seismograms are released here.

A schematic of the observational system is shown in Figure 2 indicating primary-components and signal flow. The most important differences discovered from previously used calibration values, in terms of their impact on the seismograms and inferred ground motions, are the filters' high-pass (low-cut) frequencies and the sensitivities of the accelerometers at PS11. Accelerometer sensitivities at PS11 were found to be higher by about 8\% than previously reported. Highpass filter corner frequencies have a substantial impact on long-period ground motion, and therefore on displacement records. These frequencies varied from the nominal $0.1 \mathrm{~Hz}$ by significant margins in all nine components tested and in all cases were lower. 


\section{STATION COORDINATES AND ORIENTATIONS}

We used differential GPS relative to a local benchmark to locate and measure the bearing (via laser sight line) of the three sets of accelerometers. We achieved worst-case horizontal accuracy of $1 \mathrm{~m}$, vertical accuracy of $2 \mathrm{~m}$, and azimuthal accuracy of 1.1 degrees. In most cases, accuracies were much better - benchmark uncertainty limited the accuracy of the worst case, PS11. In a subsequent journal paper we hope that all sites will be referenced to the equivalent of WGS84 (ITRF00) ellipsoid elevations (this being a reasonable choice for seismic ray-length computations), however elevations are currently referenced to a mix of older orthometric and ellipsoid datums because of the difficulties in making such conversions in this part of Alaska. In that paper we will also give NAD 27/NGVD 29 coordinates because these are the datums most widely available and used in Alaska.

Tables $1 a-c$ give the coordinates and orientations of the three strong-motion sensors, the accelerometer sets themselves. To establish the sensor azimuths, we used differential-GPS points along laser bore-sight lines pointed along the direction of stiff plastic jigs aligned to the sensors, and in the case of PS09 and PS11 with the bore sight sitting directly on the plastic jig in a Vgroove machined for the purpose. (Bore sights are small solid-state lasers normally used to align gun sights by taking the place of the cartridge.) In the case of PS10, where the plastic jig was much longer $\left(3^{\prime}, 91.4 \mathrm{~cm}\right)$ than at the other two sites $\left(1^{\prime}, 30.3 \mathrm{~cm}\right)$ the jig was further stiffened with aluminum channel stock to limit flexing in the wind. The bore-sight pointing jig was in turn held firmly to the side of the 6.1-cm-wide machined aluminum mounting block in which the accelerometers are screw mounted. Only in the case of PS10 is the seismograph vault surrounded by a "doghouse" of concrete walls three-feet high $(91.4 \mathrm{~cm})$ and six-inches thick $(15.2 \mathrm{~cm})$, walls intended to reduce snow-melt infiltration. So for PS10 we transferred the jig azimuth to a length of aluminum extrusion (an "L" section), carefully chosen for its straightness. The "L" extrusion was placed atop and across the facing walls of the doghouse and held in place there by plumber's clay. The azimuth transfer from the jig to this "L" extrusion was accomplished with a pair of plumb bobs. Given the small size of the accelerometer mounting block, disturbance by the prevailing winds, the need to hand-hold the jig to the mounting block, and various other factors, there is as much as a $1.1^{\circ}$ error in the azimuth determination at PS10. We estimate smaller errors of $0.4^{\circ}$ at PS09 and PS11, where conditions were more favorable and the bore sight sat directly 
in the $\mathrm{V}$-groove of the smaller, stiffer jig rather than in the "L" extrusion. These pointing-error estimates are sums of estimated errors from the various error sources in the chain of measurements required to produce an azimuth (for example, an estimate of the error in holding the jig to the sensor block, in aligning the bore sight to the jig, the bore sight's own accuracy, GPS errors, and so forth). Each element in the sums was made conservative and summing them is conservative, therefore the sums are maximal estimates of error.

In all cases we used differential GPS computed a posteriori to obtain an absolute position of the site itself, with the reference GPS unit at a benchmark and the roving GPS unit located just above the accelerometers and corrected for this elevation offset. At PS09 the reference benchmark is very good, excepting minor issues of permafrost. At PS10 we used a bridge-abutment benchmark recently resurveyed by APSC using their own high-quality differential systems to relocate it relative to NGS (the National Geodetic Survey) benchmarks (as part of APSC's postearthquake facilities resurveying program). We had particular problems at PS11 (Table 1c) because we and NGS later learned that the NGS benchmark we used likely had antenna-reduction problems during NGS' 1988 GPS resurvey. Therefore, PS11 has possible location errors as large

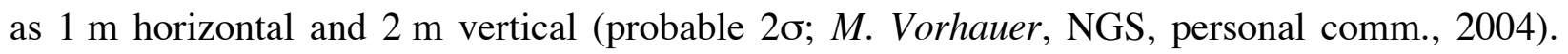
Nevertheless, this error envelope is sufficiently good for nearly all seismological applications.

With the present data, we note differences from the original APSC estimates of instrument orientations. These original estimates were based on the local "plant coordinates" and the assumption that the contractors building the instrument pads oriented them accurately (D. Nyman, personal comm., 2003). These concrete pads are 5'×5'-wide $\times 2$ '-thick $(152 \mathrm{~cm} \times 152 \mathrm{~m} \times 61$ $\mathrm{cm})$. We are also assuming no shifting of the pads during the earthquakes - there is no evidence for such changes, such as gaps between the pads and surrounding soil, either as observed by us or any earlier visitors we have contacted. 
Table 1a. Location and orientation of the accelerometers at Pump Station 09.

\begin{tabular}{|l|r|r|c|}
\hline \multicolumn{1}{|c|}{ PS9 } & \multicolumn{1}{|c|}{ Value } & Est. Error & Comments \\
\hline Latitude & $\mathbf{6 3 . 9 2 9 8 2 0 4}$ & 0.000010 & WGS84 \\
Longitude & $\mathbf{- 1 4 5 . 7 6 8 2 9 6 9}$ & 0.000023 & WGS84 \\
Elevation (m) & $\mathbf{4 6 9 . 9}$ & 0.4 & NAD83 \\
\hline "North" Azm & $\mathbf{1 4 . 9}$ & 0.4 & Notes 1,3 \\
"East" Azm & $\mathbf{1 0 4 . 9}$ & 0.4 & Notes 1,3 \\
\hline "North" Dip (deg) & 0.2 & 0.2 & Note 2 \\
"East" Dip (deg) & -0.4 & 0.2 & Note 2 \\
\hline
\end{tabular}

Table 1b. Location and orientation of the accelerometers at Pump Station 10.

\begin{tabular}{|l|r|r|c|}
\hline \multicolumn{1}{|c|}{ PS10 } & \multicolumn{1}{c|}{ Value } & Est. Error & Datum, ... \\
\hline Latitude & $\mathbf{6 3 . 4 2 4 4 9 4 4}$ & 0.0000009 & ITRF00 \\
Longitude & $\mathbf{- 1 4 5 . 7 6 2 6 6 4}$ & 0.0000020 & ITRF00 \\
Elevation (m) & $\mathbf{7 2 6 . 1}$ & 0.4 & NGVD29 \\
\hline "North" Azm & $\mathbf{3 1 6 . 7}$ & 1.1 & Notes 1,3 \\
"East" Azm & $\mathbf{4 6 . 7}$ & 1.1 & Notes 1,3 \\
\hline "North" Dip (deg) & 0.2 & 0.2 & Note 2 \\
"East" Dip (deg) & 0.2 & 0.2 & Note 2 \\
\hline
\end{tabular}

Table 1c. Location and orientation of the accelerometers at Pump Station 11.

\begin{tabular}{|l|r|r|c|}
\hline \multicolumn{1}{|c|}{ PS11 } & Value & $\begin{array}{c}\text { Est. Error } \\
\text { (Note 5) }\end{array}$ & Comments \\
\hline Latitude & $\mathbf{6 2 . 0 8 7 6 9 2}$ & 0.000010 & WGS84 \\
Longitude & $\mathbf{- 1 4 5 . 4 8 2 1 5 1}$ & 0.000022 & WGS84 \\
Elevation (m) & $\mathbf{3 9 7}$ & 0.4 & NAVD88 \\
\hline "North" Azm & $\mathbf{3 3 6 . 7}$ & 0.4 & Notes 1,4 \\
"East" Azm & $\mathbf{6 6 . 7}$ & 0.4 & Notes 1,4 \\
\hline "North" Dip (deg) & $\mathbf{1 . 3}$ & 0.2 & Note 2 \\
"East" Dip (deg) & 0.0 & 0.2 & Note 2 \\
\hline
\end{tabular}

Notes for Tables $1 a-c$ :

1) GPS positioning of points along laser bore-sight line from jig held to face of accelerometer mounting block.

2) Machinist's bubble level and large protractor. Positive dip is down.

3) Weighted average of azimuths from station to points along bore-sight line.

4) Azimuth of line fit to site position and positions of points along bore-sight line.

5) Reference benchmark has $2 \mathrm{~s}$ horizontal errors of $1 \mathrm{~m}$ and vertical error of $2 \mathrm{~m}$, which dominate site-location errors (cf., 10 and $20 \mathrm{~cm}$ errors at the other sites). 
These variations between original estimates and our new measurements range from negligible at PS11 (a $0.89^{\circ}$ difference between $336.72^{\circ}$ for the new result and $335.83^{\circ}$ for plant coordinates) to fairly large at PS10 (a $4.36^{\circ}$ difference between $316.70^{\circ}$ for the new result and $321.06^{\circ}$ for plant coordinates). At PS09 the difference was $2.20^{\circ}$ between $14.94^{\circ}$ for the new result and $12.74^{\circ}$ for plant coordinates. (These azimuths are all for the "North" components, here understood to mean those parallel to the pipeline's local azimuth with "East" components pointing orthogonal to the pipeline. We do not mean the cardinal points of the compass.) The instruments were also all found to be slightly out of level, negligibly so at PS09 and PS10 but by $1.3^{\circ} \pm 0.2^{\circ}$ for the "North" component of PS11. However, the active axis of any seismometer generally is not parallel to the case of the instrument, e.g., we later measured differences of up to $0.5^{\circ}$ between case and the active axis of these accelerometers during tilt calibrations.

Site conditions (materials velocities and damage observed) are described by Kayen et al. (2004). They give " $v_{S 30}$ " as 376,316 , and $362 \mathrm{~m} / \mathrm{s}$ (thus NEHRP classes are C, D, C) for stations PS09, PS10, and PS11.

\section{ACCELEROMETER CALIBRATIONS}

Calibrations of accelerometers with flat frequency responses are, in principal, very straightforward. One simply tilts the device at a wide range of angles, applying a fraction of the Earth's static gravitational acceleration as the input signal, and measures the resulting outputs. As long as the sensor is accurately leveled and oriented to the tilt axis of the table, the sine of a wellmeasured input tilt angle is the input acceleration (fraction of local value of g). Some complications of this simple sounding procedure at this level of precision include orienting the active axis of the sensor (not the sensor case) to the table while the table is tilted about $30^{\circ}$ such that output is maximized as measured on a very precise digital voltmeter (DVM) - a matter of considerable dexterity and patience. Failing to make this alignment accurately yields a roughly half-sine perturbation at least as large as other uncertainties to the measured values. As alluded, it is also somewhat important (several parts per thousand) to note the local value of gravity at the test location and correct for it. Figure 3 shows the results for such a calibration of the three components at PS10, giving the voltage output of the accelerometers' amplifier versus the acceleration applied. While the slopes of these lines are the key results here, this plot is not particularly in- 
formative because the voltage scale is coarse. Residuals to these maximum likelihood fits are much more helpful.

\section{Accelerometer Sensitivity at PS10}

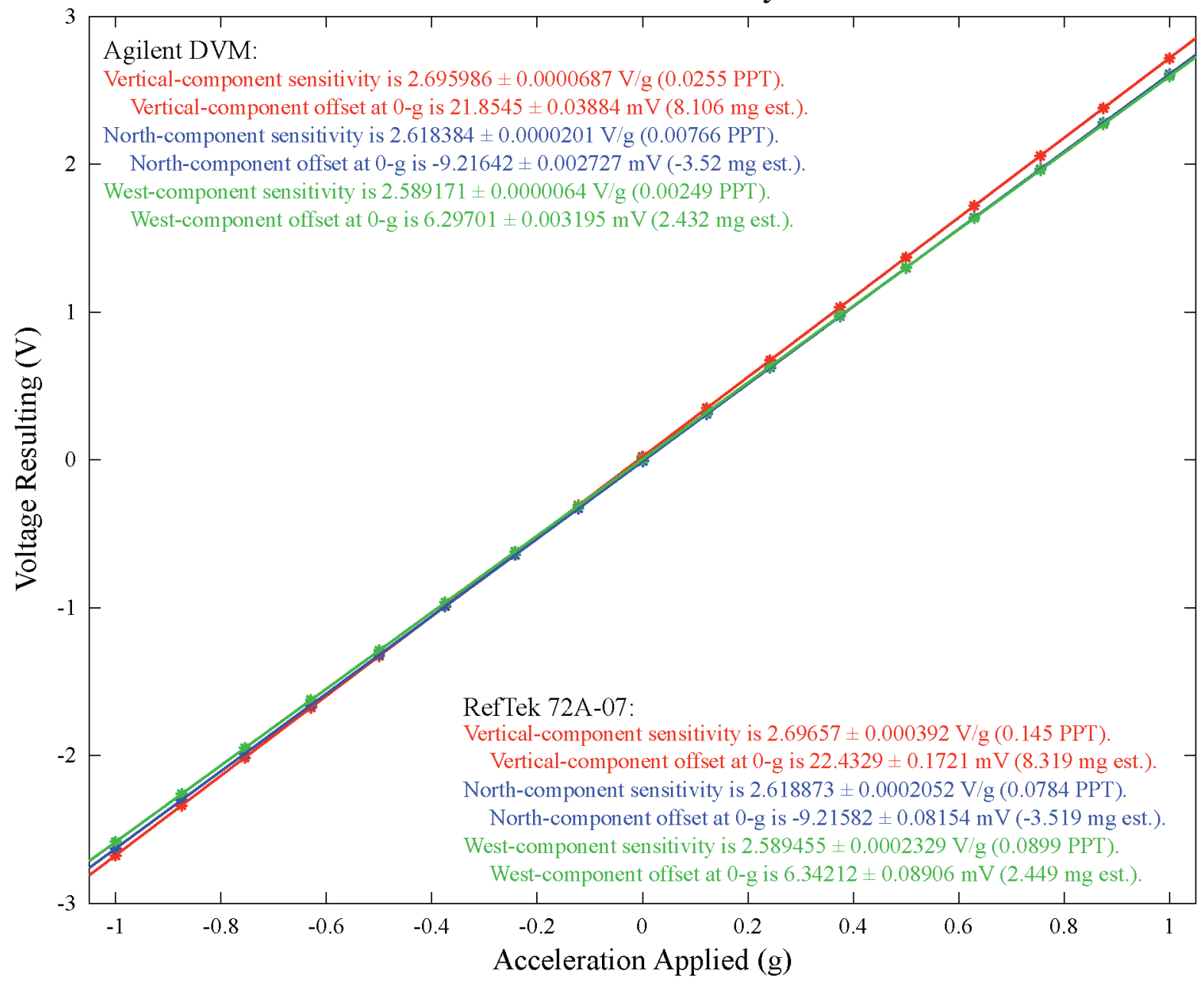

Figure 3. Raw data and maximum likelihood fits for the three accelerometers of station PS10. The two data sets were measured directly with an Agilent ${ }^{\mathrm{TM}}$ 34907A integrating digital voltmeter (DVM) and a purpose-calibrated RefTek ${ }^{\mathrm{TM}} 72 \mathrm{~A}-02$ recorder. The former appear to be slightly more precise.

Figures $4 a-c$ show the residuals for the calibrations of all nine accelerometers, three components at three sites. Tables $2 a-c$ summarize the sensitivities obtained from these tests, their accuracies, and the sensitivities reported previously by APSC. Six components are in agreement to about $0.5 \%$, but those at PS11 are 8-9\% higher than previous values. The current values have been verified several times and in a number of ways and are not affected by this previous error. 
Table 2a. Sensitivities of the accelerometers at PS09 by laboratory test (corrected for resistor values). Preferred values are in boldface. Accuracy is limited in this one case by amplifier resistor values, known to $4 \frac{1}{2}$ places from a well-calibrated meter. The upper block of values is relative to local $g$ while the lower three lines are corrected for this variable (bottom line is sensitivity in ICWM $g$ 's, $980.665 \mathrm{~cm} / \mathrm{s}^{2}$ ).

\begin{tabular}{|c|c|c|c|c|c|c|}
\hline \multirow{2}{*}{ Source } & \multicolumn{3}{|c|}{ Sensitivity (Volts/g) ${ }^{*}$} & \multicolumn{3}{|c|}{ Ratio of USGS / APSC ${ }^{* *}$} \\
\hline & Vertical & "North" & "East" & Vertical & "North" & "East" \\
\hline APSC & 2.869 & 2.672 & 2.548 & 1 & 1 & 1 \\
\hline Agilent $^{\mathrm{TM}}$ & 2.8506 & 2.6585 & 2.5321 & 0.996 & 0.997 & 0.996 \\
\hline RefTek $^{\text {TM }}$ & 2.8504 & 2.6594 & 2.5326 & 0.996 & 0.997 & 0.996 \\
\hline \multirow{2}{*}{ Source } & \multicolumn{3}{|c|}{ Sensitivity (mV/gal) } & \multicolumn{3}{|c|}{ Standard Deviation } \\
\hline & Vertical & "North" & "East" & Vertical & "North" & "East" \\
\hline Agilent $^{\text {TM }}$ & 2.9090 & 2.7129 & 2.5839 & 0.00001 & 0.00004 & 0.00005 \\
\hline RefTek $^{\text {TM }}$ & 2.9087 & 2.7139 & 2.5844 & 0.00030 & 0.00028 & 0.00022 \\
\hline \multirow[b]{2}{*}{ Agilent $^{\mathrm{TM}}$} & \multicolumn{3}{|c|}{ Sensitivity (Volts per standard g) } & \multicolumn{3}{|c|}{ Standard Deviation } \\
\hline & 2.85271 & 2.66041 & 2.53394 & 0.00001 & 0.00004 & 0.00005 \\
\hline
\end{tabular}

Table $2 \boldsymbol{b}$. Sensitivities of the accelerometers at PS10 by precision laboratory test. Final, preferred values are in boldface. Comparison ratios to original APSC values are in the upper right.

\begin{tabular}{|c|ccc|ccc|}
\hline \multirow{2}{*}{ Source } & \multicolumn{3}{|c|}{ Sensitivity (Volts/g)* } & \multicolumn{3}{c|}{ USGS / APSC * * $^{*}$} \\
\cline { 2 - 7 } & Vertical & "North" & "East" & Vertical & "North" & "East" \\
\hline APSC & 2.708 & 2.630 & 2.596 & 1 & 1 & 1 \\
Agilent $^{\mathrm{TM}}$ & 2.69599 & 2.61838 & 2.58917 & 0.998 & 0.998 & 1.000 \\
RefTek $^{\mathrm{TM}}$ & 2.69657 & 2.61887 & 2.58946 & 0.998 & 0.998 & 1.000 \\
\hline \multirow{2}{*}{ Source } & \multicolumn{2}{|c|}{ Sensitivity (mV/gal) } & \multicolumn{3}{c|}{ Standard Deviation } \\
\cline { 2 - 7 } & Vertical & "North" & "East" & Vertical & "North" & "East" \\
\hline Agilent $^{\mathrm{TM}}$ & $\mathbf{2 . 7 5 1 1 6}$ & $\mathbf{2 . 6 7 1 9 7}$ & $\mathbf{2 . 6 4 2 1 6}$ & 0.00007 & 0.00002 & 0.00001 \\
RefTek $^{\mathrm{TM}}$ & 2.75176 & 2.67247 & $\mathbf{2 . 6 4 2 4 5}$ & 0.00040 & 0.00021 & 0.00024 \\
\hline Preferred, & \multicolumn{2}{|c|}{ Sensitivity (Volts per standard g) } & \multicolumn{3}{c|}{ Standard Deviation } \\
\cline { 2 - 7 } Agilent $^{\mathbf{T M}}$ & $\mathbf{2 . 6 9 7 9 7}$ & $\mathbf{2 . 6 2 0 3 1}$ & $\mathbf{2 . 5 9 1 0 7}$ & \multicolumn{4}{c|}{0.00007} & 0.00002 & 0.00001 \\
\hline
\end{tabular}

Table 2c. Like Table $2 b$ but for PS11.

\begin{tabular}{|c|c|c|c|c|c|c|}
\hline \multirow{2}{*}{ Source } & \multicolumn{3}{|c|}{ Sensitivity (Volts/g) ${ }^{*}$} & \multicolumn{3}{|c|}{ USGS / APSC** } \\
\hline & Vertical & "North" & "East" & Vertical & "North" & "East" \\
\hline APSC & 2.651 & 2.708 & 2.485 & 1 & 1 & 1 \\
\hline Agilent $^{\mathrm{TM}}$ & 2.85889 & 2.91612 & 2.70068 & 1.081 & 1.079 & 1.089 \\
\hline RefTek $^{\text {TM }}$ & 2.85871 & 2.91634 & 2.70132 & 1.080 & 1.079 & 1.089 \\
\hline \multirow{2}{*}{ Source } & \multicolumn{3}{|c|}{ Sensitivity (mV/gal) } & \multicolumn{3}{|c|}{ Standard Deviation } \\
\hline & Vertical & "North" & "East" & Vertical & "North" & "East" \\
\hline Agilent $^{\mathrm{TM}}$ & 2.91740 & 2.97580 & 2.75595 & 0.00001 & 0.00001 & 0.00007 \\
\hline RefTek $^{\text {TM }}$ & 2.91722 & 2.97602 & 2.75660 & 0.00032 & 0.00027 & 0.00028 \\
\hline \multirow{2}{*}{$\begin{array}{l}\text { Preferred, } \\
\text { Agilent }^{\mathrm{TM}}\end{array}$} & \multicolumn{3}{|c|}{ Sensitivity (Volts per standard g) } & \multicolumn{3}{|c|}{$\begin{array}{l}\text { Standard Deviation } \\
\end{array}$} \\
\hline & 2.86099 & 2.91826 & 2.70266 & 0.00001 & 0.00001 & 0.00007 \\
\hline
\end{tabular}

Notes for Tables $2 a-c$ : *Local value of gravity at Menlo Park is $979.945 \pm 0.002$ gal (NGS "Surface Gravity Prediction" model). For other uses please use the value referenced standard-g (980.665 gal), as shown in the bottom rows. **Ratio has been corrected to gravity value at APSC site. 
Table 2d. Sensitivities of the accelerometers at PS09 derived from a field test using the correct amplifier and $1.91 \mathrm{KW}$ resistors. The largest difference from the values in Table $2 a$ is $3.1 \mathrm{PPT}$, about the estimated error range of the values given here.

\begin{tabular}{|c|c|c|c|c|c|c|}
\hline \multirow{2}{*}{ Source } & \multicolumn{3}{|c|}{ Sensitivity (Volts/g)* } & \multicolumn{3}{|c|}{ Table $2 d / 2 a$} \\
\hline & Vertical & "North" & "East" & Vertical & "North" & "East" \\
\hline APSC & 2.869 & 2.672 & 2.548 & & & \\
\hline Fluke ${ }^{\mathrm{TM}}$ & 2.8543 & 2.6592 & 2.5397 & 1.0013 & 1.0003 & 1.0030 \\
\hline RefTek $^{\mathrm{TM}}$ & 2.8546 & 2.6601 & 2.5405 & 1.0015 & 1.0002 & 1.0031 \\
\hline \multirow{2}{*}{ Source } & \multicolumn{3}{|c|}{ Sensitivity $(\mathrm{mV} /$ gal $)$} & & & \\
\hline & Vertical & "North" & "East" & & & \\
\hline RefTek $^{\text {TM }}$ & 2.9068 & 2.7087 & 2.5869 & & & \\
\hline \multirow{2}{*}{$\begin{array}{l}\text { Preferred, } \\
\text { Agilent }^{\mathrm{TM}}\end{array}$} & \multicolumn{3}{|c|}{ Sensitivity (Volts per standard g) } & & & \\
\hline & 2.85056 & 2.65632 & 2.53688 & & & \\
\hline
\end{tabular}

For PS09, because its amplifier had to be returned to APSC for use in the field, we had to use a buffer amplifier with an incorrect set of $1.91 \mathrm{KW}$ resistors (those of PS10). These resistors are used to convert the accelerometers' output from a current proportional to acceleration into a voltage proportional to acceleration. The conversion resistors in question are $1 \%$ metal-film type so they introduce about a $1 \%$ uncertainty (probably less because the batch of resistors from which these came showed less variation). (They are not the source of the $8 \%$ difference in sensitivity between APSC and USGS calibrations at PS11.) Nevertheless, in generating Table $2 a$, for PS09 it was necessary to measure the resistors in both the PS10 and PS09 amplifiers and correct for this slight discrepancy. Indeed, the measurement of the resistors for PS09, necessarily taken in the field to only $4 \frac{1}{2}$ digits, is the limiting precision factor in Table $2 a$.

Using a notation equivalent to that introduced below (in SIGNAL FLOW AND GOVERNING EQUATIONS), we make this resistor correction very simply by $V_{\text {test }}$ $=\left(R_{\mathrm{PS} 09} / R_{\mathrm{PS} 10}\right)\left(R_{\mathrm{PS} 10} S_{\mathrm{mA}}\right) a_{\text {test }}$, where $V_{\text {test }}$ is the corrected voltage out of the amplifier for test acceleration $a_{t e s t}$, as corrected for the differing resistors. The measured voltage was $\left(R_{\mathrm{PS} 10} S_{\mathrm{mA}}\right) a_{\text {test }}$ while the corrected value was $\left(R_{\mathrm{PS} 09} S_{\mathrm{mA}}\right) a_{\text {test }}$.

To rule out large errors, we also supply sensitivity values we obtained during tests in the field using the correct PS09 amplifier and resistors but a less precise portable tilt table. These are given in Table $2 d$ and have accuracies of about three parts per thousand (3 PPT), or better. Ratios between Tables $2 a$ and $2 d$ are, indeed, no larger than 3 PPT. (This portable tilt table, of metal-stiffened acrylic, was a simple piano-hinged flap held at a fixed set of angles from 0-90 by the attachment of machined aluminum bars near either corner. It's base was leveled by using 
large bolts ground to a point as support legs. Accuracy was limited by flexing, leveling issues on slippery concrete, and the precision and wear of the aluminum bars. The more precise laboratory table was a rotary machine-tool stage, Enco $^{\text {TM }}$ 200-1163, equipped with a precision right-angle plate and accurate to no worse than $\pm 45 \operatorname{arcsec}, 0.4$ PPT at $30^{\circ}$ tilt.)

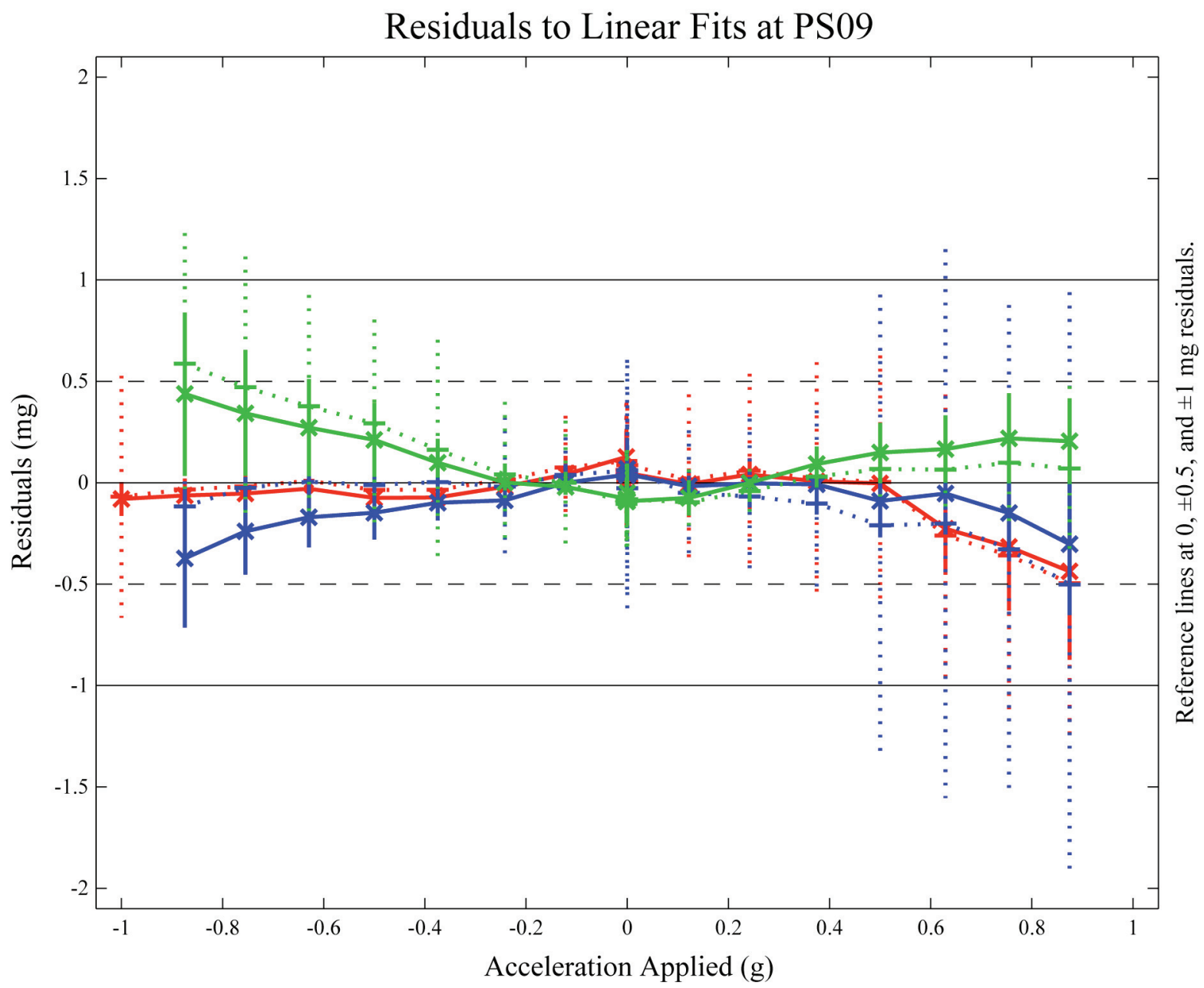

Figure 4a. Residuals from linear maximum likelihood fits to static tilt calibrations of the three accelerometers at PS09. Red = vertical; blue = "North"; green = "East" component. Solid lines and crosses are values obtained from the Agilent ${ }^{\mathrm{TM}}$ 34907A integrating DVM; dotted lines and plus signs are values obtained by taking means of selected intervals of 200-sps time series from a RefTek ${ }^{\mathrm{TM}} 72 \mathrm{~A}-07 / 6$ 24-bit recorder, which we calibrated for the purpose. Vertical bars are corresponding $\pm 2 \mathrm{~s}$ ranges. (The zeroacceleration points were measured before and after the other values, so there are twice as many points there.) 


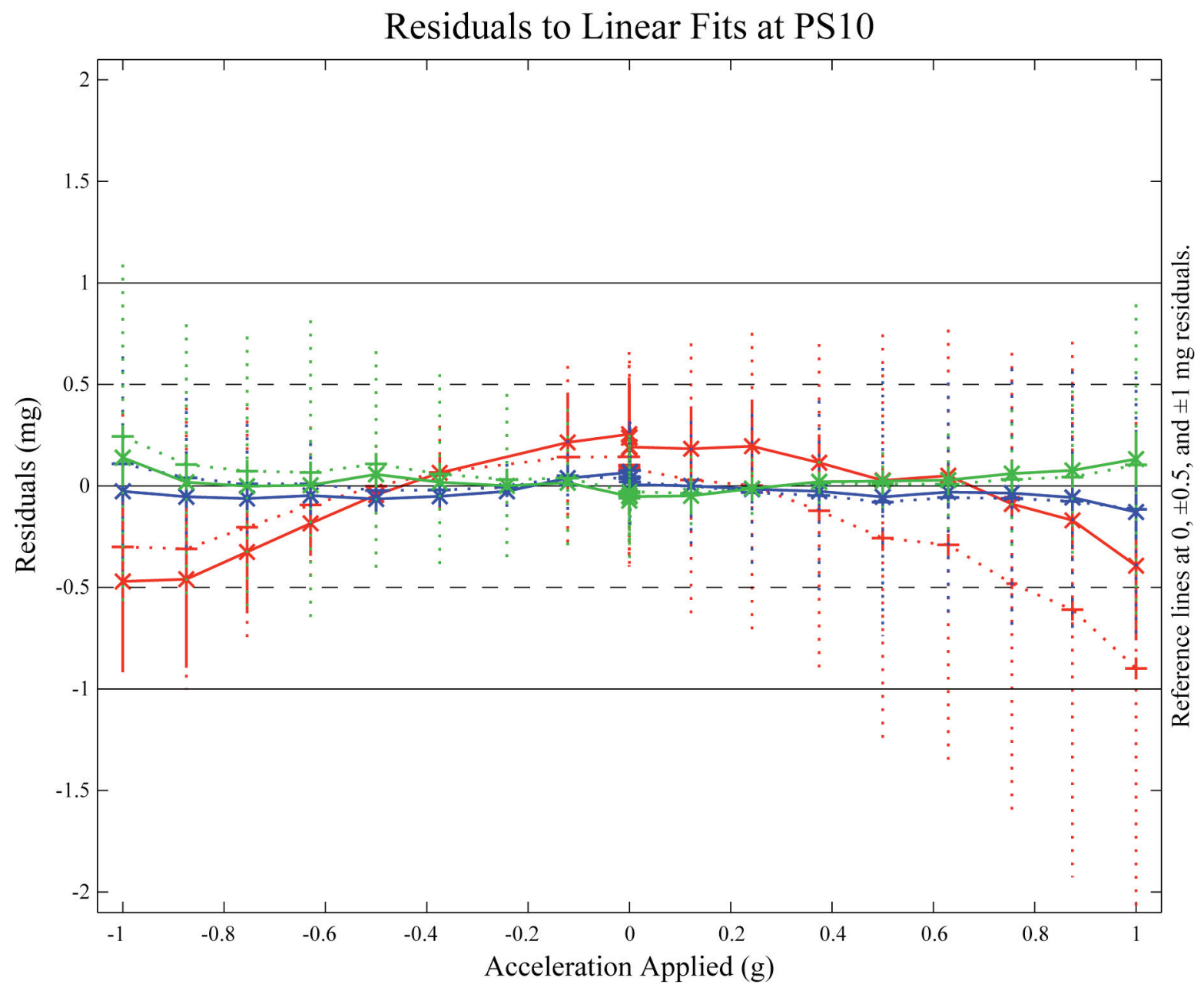

Figure $4 b$. Like Figure $4 a$ but for PS10.

\section{SOME DETAILS}

We measured individual voltages from the accelerometers in two ways, with a $61 / 2$-digit (\$22bit) Agilent ${ }^{\mathrm{TM}}$ 34907A integrating DVM, and with a carefully calibrated RefTek ${ }^{\mathrm{TM}}$ 72A-07/6 "24-bit" seismograph. (It is essential to calibrate such seismographs when using them in calibration work, as discussed below.) We used the RefTek ${ }^{\mathrm{TM}}$ at $200 \mathrm{sps}$ (samples per second) to be able to review data quality and noise factors more thoroughly than with the Agilent ${ }^{\mathrm{TM}}$. However, the DVM, because it integrates across a precise number of power-line cycles (100 in this case), notch-filters out the worst single source of noise in the test, and produced lower standard deviations in the raw measurements (generally by a factor on 100 to 300). 


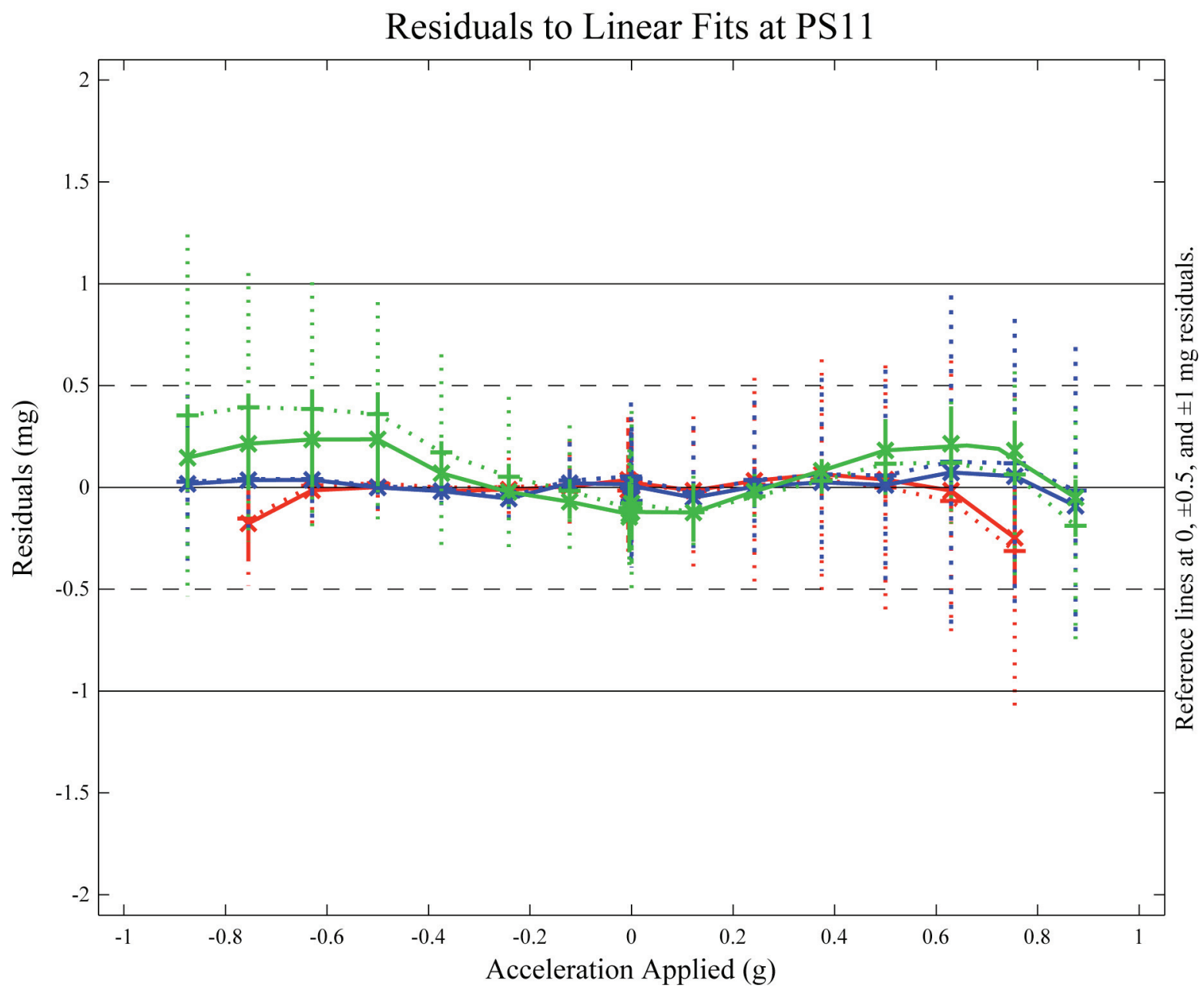

Figure 4c. Like Figure $4 a$ but for PS11.

The Agilent ${ }^{\mathrm{TM}}$ DVM also produced better fits in our two-step maximum likelihood linearregression method. Nevertheless, we note that residuals between the best-fitting line and the data uniformly exceed measurement standard deviations by several orders of magnitude, typically by four orders. Thus, there are sources of error in the system that greatly outweigh measured timeseries noise (the sum of electronic noise, cultural noise, and sensor self noise), so that our best (indeed only) available estimates of these larger errors are the residuals to the linear fits. To obtain reasonable estimates of the standard deviations of the slope of the fitted line, we must give the maximum likelihood method reasonable estimates of the standard deviations of the measurements. Therefore, we first fit those data using the (tiny) standard deviations of the voltages themselves, then use the absolute values of the residuals to this first fit as estimates of twice the true standard deviations of the data and perform a second fit to the data, using these new, much larger (in all but one case) standard deviations for the second fit. We believe that using the re- 
siduals as $95 \%$ estimates of uncertainty for the input data provides a more realistic estimate for uncertainties in the slope of the fit line. And, of course, that slope is the measure we seek of the accelerometer's sensitivity.

\section{CALIBRATION OF THE REFTEK ${ }^{\text {TM }}$ SEISMOGRAPH}

The RefTek ${ }^{\mathrm{TM}}$ ADC conversion together with its associated amplifier gain coefficients have been individually calibrated against a Datel ${ }^{\mathrm{TM}}$ DVC-8500A voltage reference and also the Agilent $^{\mathrm{TM}}$ 34907A 61/2-digit DVM. The Datel ${ }^{\mathrm{TM}}$ and Agilent ${ }^{\mathrm{TM}}$ agreed within $0.5 \mathrm{mV}$ up to $\pm 9 \mathrm{VDC}$ (that is, to one part in 18,000). We adjusted the vernier setting on the Datel ${ }^{\mathrm{TM}}$ to make it agree even more closely with the Agilent ${ }^{\mathrm{TM}}$ because the latter has been NIST-traceably calibrated much more recently. Therefore, the Agilent ${ }^{\mathrm{TM}}$ is the master reference standard for all these tests. Finally, we recorded segments of signal from the Datel ${ }^{\mathrm{TM}}$ on the RefTek ${ }^{\mathrm{TM}}$ and evaluated them to determine the RefTek ${ }^{\mathrm{TM}}$ 's ADC conversion coefficients.

We found that the RefTek ${ }^{\mathrm{TM}}$ 's individual channels varied by up to $0.6 \%$ from the nominal calibration factor in the data headers for the two model 72A-07/6 recorders we tested, a total of 12 channels. Calibration factors also varied by as much as $0.7 \%$ between channels within a single recorder (plus a very much smaller temporal change likely due to temperature variations). Finally, over the 180-s duration of each calibration record we noted tiny, but measurable drift, typically 15 to $25 \mu \mathrm{V}$ peak-to-peak in the case of recorder $\# 7880$ and $10 \mu \mathrm{V}$ peak-to-peak for recorder \#7871. (One count is about $1.9 \mu \mathrm{V}$ at this unity gain setting and $200-\mathrm{Hz}$ sample rate.) The shape of the drift was partly unique to each channel but shared common characteristics within groups of three channels (1-3 and 4-6), presumably because these groups share many common electronic parts. Because of imprecision in amplifier gains (the resistors are $0.1 \%$ accurate), temperature sensitivities, and other factors, modern seismographs have remarkable relative accuracy yet still lack the high absolute accuracy required for precise calibration work unless they are themselves calibrated for the purpose.

Similarly, the ability of sampling DVMs to synchronize with the local power lines in order to filter noise sources synchronous with those frequencies makes high-resolution sampling, integrating DVMs the instrument of choice in many types of calibration work. High-speed sampling DVMs are available that can provide the bandwidth advantages of a modern seismograph as well 
but only for a single channel. Thus the principal exception is in tests requiring precise relative timing between multiple channels, such as tests for non-coherent noise (self-noise when a location quieter than the sensor cannot be arranged).

\section{FILTER CALIBRATIONS}

Excepting the sensor gain at PS11, the bandpass filters (Tables 3a-c) are the most significant contributors to differences in system performance from the nominal specifications available during the first year after the earthquake. Those original specifications were for a two-pole highpass Butterworth filter with a -3-db cut-off at $0.1 \mathrm{~Hz}$ followed by a two-pole low-pass Butterworth filter with a -3 -db cut-off at $40.0 \mathrm{~Hz}$, and total filter gain (out/in ratio) of $2.51 \times$. We measured the actual filter responses using a Hewlett Packard HP3562A ${ }^{\mathrm{TM}}$ Dynamic Signal Analyzer.

As one would expect with real components, neither section is precisely a true Butterworth filter (the poles do not lie on a zero-centered circle in the complex $z$ plane), although this distinction is not large. More significantly, the corner frequencies are not as intended. Most importantly for the deconvolved seismograms, the -3-db points of the high-pass 2-pole sections (interpolated in the frequency domain for the Tables) all fall well below $0.1 \mathrm{~Hz}$. These differences have significant impacts on the long-period responses of the instruments, which affect computed velocities and displacements since these are strongly dependent on long-period signal content. For example, at PS10 the -3 -db corners are closer to $0.086 \mathrm{~Hz}$ than to $0.1 \mathrm{~Hz}$ (Table $3 b$ ), which changes displacements roughly 5\% (Ellsworth et al., 2004).

Because the filters are not strictly Butterworth, direct simulation of individual filters rather than parametric representation as Butterworth filters is preferred when deconvolving instrument responses. Each component of each station certainly must be treated independently with its individual response characteristics. 
Table 3a. Laplace filter coefficients and Butterworth equivalent corner frequencies at PS09. (Stage $1 \approx$ 2-pole Butterworth high-pass with $0.087 \pm 0.001-\mathrm{Hz}-3$-db cutoff and gain of $1.596 \pm 0.002$. Stage $2 \approx 2$ pole Butterworth low-pass with $40.3 \pm 0.8-\mathrm{Hz}-3$-db cutoff and gain of $1.595 \pm 0.002$. Poles are in radians per second and gains are direct out/in ratios.) Total gain factors are given at $2.0 \mathrm{~Hz}$.

\begin{tabular}{|c|c|c|c|c|c|c|c|c|c|}
\hline \multirow{3}{*}{ Site } & \multirow{3}{*}{ Channel } & \multicolumn{8}{|c|}{ Stage 1 ( $\sim 0.1-\mathrm{Hz}$ High-Pass) } \\
\hline & & \multicolumn{2}{|c|}{ Pole 1 $\left(\mathrm{s}_{\mathrm{H} 1}\right)$} & \multicolumn{2}{|c|}{ Pole $2\left(\mathrm{~s}_{\mathrm{H} 2}\right)$} & \multirow{2}{*}{ Zero 1} & \multirow{2}{*}{ Zero 2} & \multirow{2}{*}{ Gain } & \multirow{2}{*}{$\begin{array}{c}-3 \mathrm{~dB} \\
(\mathrm{~Hz})\end{array}$} \\
\hline & & Real & Imag & Real & Imag & & & & \\
\hline \multirow{3}{*}{ PS09 } & Vertical (2) & -0.38242 & 0.38011 & -0.38242 & -0.38011 & $0+0 \mathrm{i}$ & $0-0 \mathrm{i}$ & 1.5982 & 0.08630 \\
\hline & "North" (1) & -0.38247 & 0.37100 & -0.38247 & -0.37100 & $0+0 \mathrm{i}$ & $0-0 \mathrm{i}$ & 1.5952 & 0.08633 \\
\hline & "East" (3) & -0.38970 & 0.37194 & -0.38970 & -0.37194 & $0+0 \mathrm{i}$ & $0-0 \mathrm{i}$ & 1.5957 & 0.08856 \\
\hline
\end{tabular}

\begin{tabular}{|c|c|c|c|c|c|c|c|c|}
\hline \multirow{3}{*}{ Site } & \multirow{3}{*}{ Channel } & \multicolumn{6}{|c|}{ Stage 2 ( $40-\mathrm{Hz}$ Low-Pass) } & \multirow{3}{*}{$\begin{array}{l}\text { Total } \\
\text { Filter } \\
\text { Gain }\end{array}$} \\
\hline & & \multicolumn{2}{|c|}{ Pole $1\left(\mathrm{~s}_{\mathrm{L} 1}\right)$} & \multicolumn{2}{|c|}{ Pole $2\left(\mathrm{~s}_{\mathrm{L} 2}\right)$} & \multirow{2}{*}{ Gain } & \multirow{2}{*}{$\begin{array}{c}-3 \mathrm{~dB} \\
(\mathrm{~Hz})\end{array}$} & \\
\hline & & Real & Imag & Real & Imag & & & \\
\hline \multirow{3}{*}{ PS09 } & Vertical (2) & -178.01 & 183.34 & -178.01 & -183.34 & 1.5966 & 41.12 & 2.5517 \\
\hline & "North" (1) & -177.69 & 180.45 & -177.69 & -180.45 & 1.5944 & 40.29 & 2.5434 \\
\hline & "East" (3) & -181.71 & 175.53 & -181.71 & -175.53 & 1.5933 & 39.51 & 2.5424 \\
\hline
\end{tabular}

Table $3 \boldsymbol{b}$. Similar to Table $3 a$ but for PS10. (Stage $1 \approx 0.086 \pm 0.001 \mathrm{~Hz}-3$-db cutoff and gain of $1.597 \pm 0.002$. Stage $2 \approx 39.7 \pm 0.7 \mathrm{~Hz}-3$-db cutoff and gain of $1.592 \pm 0.002$.)

\begin{tabular}{|c|c|c|c|c|c|c|c|c|c|}
\hline \multirow{3}{*}{ Site } & \multirow{3}{*}{ Channel } & \multicolumn{8}{|c|}{ Stage $1(\sim 0.1-\mathrm{Hz}$ High-Pass $)$} \\
\hline & & \multicolumn{2}{|c|}{ Pole $\left(\mathrm{s}_{\mathrm{H} 1}\right)$} & \multicolumn{2}{|c|}{ Pole $2\left(\mathrm{~S}_{\mathrm{H} 2}\right)$} & \multirow{2}{*}{ Zero 1} & \multirow{2}{*}{ Zero 2} & \multirow{2}{*}{ Gain } & \multirow{2}{*}{$\begin{array}{l}-3 \mathrm{~dB} \\
(\mathrm{~Hz})\end{array}$} \\
\hline & & Real & Imag & Real & Imag & & & & \\
\hline \multirow{3}{*}{ PS10 } & Vertical (2) & -0.38179 & 0.37188 & -0.38179 & -0.37188 & $0+0 \mathrm{i}$ & $0-0 \mathrm{i}$ & 1.5968 & 0.08619 \\
\hline & "North" (1) & -0.38598 & 0.37076 & -0.38598 & -0.37076 & $0+0 \mathrm{i}$ & $0-0 \mathrm{i}$ & 1.5953 & 0.08711 \\
\hline & "East" (3) & -0.38209 & 0.36884 & -0.38209 & -0.36884 & $0+0 \mathrm{i}$ & $0-0 \mathrm{i}$ & 1.5990 & 0.08617 \\
\hline \multirow{3}{*}{ Site } & \multirow{3}{*}{ Channel } & \multicolumn{6}{|c|}{ Stage 2 ( $\sim 40-\mathrm{Hz}$ Low-Pass) } & & \multirow{3}{*}{$\begin{array}{c}\text { Total } \\
\text { Filter } \\
\text { Gain }\end{array}$} \\
\hline & & \multicolumn{2}{|c|}{ Pole $1\left(\mathrm{~s}_{\mathrm{L} 1}\right)$} & \multicolumn{2}{|c|}{ Pole $2\left(\mathrm{~s}_{\mathrm{L} 2}\right)$} & \multirow{2}{*}{ Gain } & \multirow{2}{*}{$\begin{array}{c}-3 \mathrm{~dB} \\
(\mathrm{~Hz}) \\
\end{array}$} & & \\
\hline & & Real & Imag & Real & Imag & & & & \\
\hline \multirow{3}{*}{ PS10 } & Vertical (2) & -183.70 & 174.02 & -183.70 & -174.02 & 1.5900 & 39.12 & & 2.5390 \\
\hline & "North" (1) & -180.97 & 178.79 & -180.97 & -178.79 & 1.5931 & 40.46 & & 2.5414 \\
\hline & "East" (3) & -179.26 & 176.13 & -179.26 & -176.13 & 1.5941 & 39.61 & & 2.5489 \\
\hline
\end{tabular}

Table 3c. Similar to Table $3 a$ but for PS11. (Stage $1 \approx 0.087 \pm 0.001 \mathrm{~Hz}-3$-db cutoff and gain of $1.597 \pm 0.003$. Stage $2 \approx 39.9 \pm 1.1 \mathrm{~Hz}-3$-db cutoff and gain of $1.594 \pm 0.001$.)

\begin{tabular}{|c|c|c|c|c|c|c|c|c|c|}
\hline \multirow{3}{*}{ Site } & \multirow{3}{*}{ Channel } & \multicolumn{8}{|c|}{ 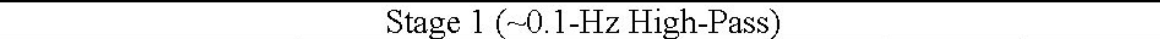 } \\
\hline & & \multicolumn{2}{|c|}{ Pole 1 $\left(\mathrm{s}_{\mathrm{H} 1}\right)$} & \multicolumn{2}{|c|}{ Pole $2\left(\mathrm{~s}_{\mathrm{H} 2}\right)$} & \multirow{2}{*}{ Zero 1} & \multirow{2}{*}{ Zero 2} & \multirow{2}{*}{ Gain } & \multirow{2}{*}{$\begin{array}{c}-3 \mathrm{~dB} \\
(\mathrm{~Hz})\end{array}$} \\
\hline & & Real & Imag & Real & Imag & & & & \\
\hline \multirow{3}{*}{ PS11 } & Vertical (2) & -0.38544 & 0.36952 & -0.38544 & -0.36952 & $0+0 \mathrm{i}$ & $0-0 \mathrm{i}$ & 1.5997 & 0.08692 \\
\hline & "North" (1) & -0.38080 & 0.37062 & -0.38080 & -0.37062 & $0+0 \mathrm{i}$ & $0-0 \mathrm{i}$ & 1.5973 & 0.08609 \\
\hline & "East" (3) & -0.38707 & 0.36995 & -0.38707 & -0.36995 & $0+0 \mathrm{i}$ & $0-0 \mathrm{i}$ & 1.5940 & 0.08735 \\
\hline
\end{tabular}

\begin{tabular}{|c|c|c|c|c|c|c|c|c|}
\hline \multirow{3}{*}{ Site } & \multirow{3}{*}{ Channel } & \multicolumn{6}{|c|}{ Stage 2 ( $\sim 40-\mathrm{Hz}$ Low-Pass) } & \multirow{3}{*}{$\begin{array}{l}\text { Total } \\
\text { Filter } \\
\text { Gain }\end{array}$} \\
\hline & & \multicolumn{2}{|c|}{ Pole $1\left(s_{L 1}\right)$} & \multicolumn{2}{|c|}{ Pole $2\left(\mathrm{~s}_{\mathrm{L} 2}\right)$} & \multirow{2}{*}{ Gain } & \multirow{2}{*}{$\begin{array}{c}-3 \mathrm{~dB} \\
(\mathrm{~Hz})\end{array}$} & \\
\hline & & Real & Imag & Real & Imag & & & \\
\hline \multirow{3}{*}{ PS11 } & Vertical (2) & -188.79 & 170.91 & -188.79 & -170.91 & 1.5934 & 38.66 & 2.5490 \\
\hline & "North" (1) & -181.61 & 181.04 & -181.61 & -181.04 & 1.5939 & 40.47 & 2.5460 \\
\hline & "East" (3) & -185.02 & 179.45 & -185.02 & -179.45 & 1.5955 & 40.52 & 2.5432 \\
\hline
\end{tabular}


(The channel "numbers" in parentheses in Tables $3 a-c$ are not sequential, with the Vertical and "North" components in reverse order. This "North"-first channel order is used internally by the APSC instruments. We have used name labels throughout to avoid confusion between USGS and APSC component-order convention, notwithstanding that "North" is to be understood as APSC "Plant coordinates" only, neither geodetic nor magnetic. Indeed, the channel numbering in Tables 3a-c may be the source of a typesetting error in Ellsworth et al. (2004) - the filter Tables in this Report are correct and should be used in preference.)

\section{BASELINE STABILITY AND SENSOR DOUBLE INTEGRATION TESTS}

We also tested both the noise characteristics of the sensors, specifically that noise's impact on baseline stability when doubly integrating the acceleration signal to recover displacement. Naturally, this effort also tests the sensors' ability to recover a known displacement accurately and tests the sensors' ability to track dynamic signals with precision in general. If the sensor could not do all these things - have low noise, stable baseline, and track a dynamic signal faithfully - it could not perform well in this test.

This test involves moving the accelerometer through a step in displacement and doubly integrating the resulting signal in an attempt to recover this known displacement step.

Figures $5 a-c$ show these results, with the displacement traces from 36 test signals, four step transits for each of the three components for each of the three instruments. Each transit is a 6.00-inch $(15.24-\mathrm{cm})$ vertical step input signal, significantly smaller than displacements at PS10 but large enough for this test. A vertical step is used because the accelerometer is far less sensitive to wobbling - errors in tilt - than if the transit were attempted in a horizontal plane. Sensitivity to gravity is according to the cosine of the angle when vertical and the sine when horizontal. This is a test previously recommended by the Advanced National Seismic System (ANSS $T I C, 2002)$.

We used a "step jig" that held the accelerometer rigidly vertical and moved it through the step either up or down (the jig is just a parallelogram of thick Acrylic, with steel reinforcements and hinges, that partially folds and unfolds to rigid stops). The size of the step is known quite accurately. We let the jig and accelerometer sit in the up or down position for three minutes be- 
fore and after each transit motion. The transit time was about $5 \mathrm{~s}$, approximating the rupture duration of the Denali-fault earthquake in November 2002.

The resulting records were $375 \mathrm{~s}$ long - we used the full length of each record, excepting the transit interval, to establish and remove the baseline by subtracting the mean of the record. We call this demeaning the "first-order" baseline correction, following the usage of several previous authors, and differing from them only in the greater length of the window used here.

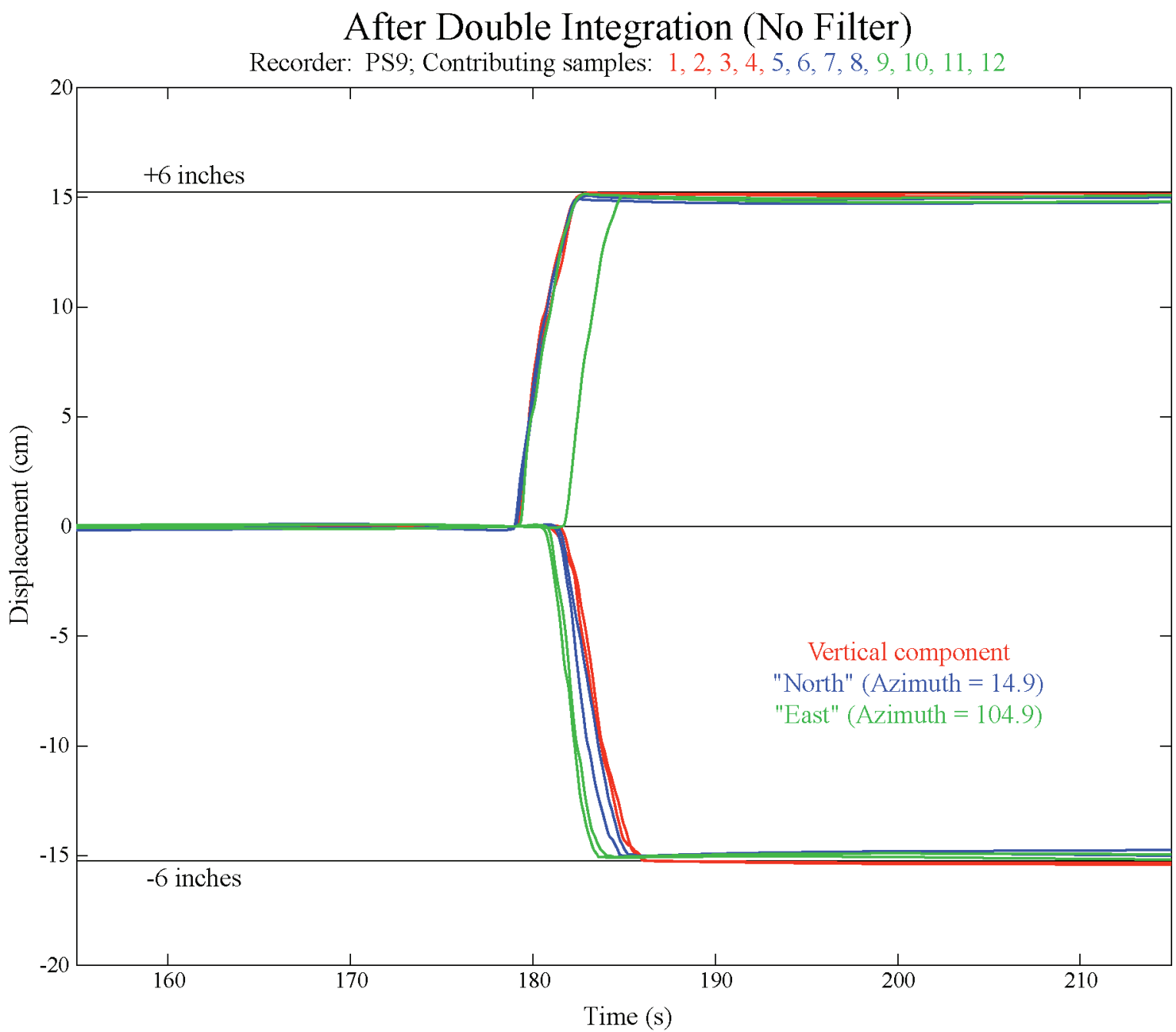

Figure 5a. Displacement traces derived by doubly integrating baseline-corrected test accelerograms from the PS09 instrument. Each component was driven through 6.00-inch $(15.24-\mathrm{cm})$ upward or downward vertical displacements, with the active axis held rigidly vertical.

Only a 60-s window in the center of this 375-s record was subjected to integration. Additionally, we used this 60-s integration window for a "second-order" baseline correction, again following several earlier authors, in which we integrate to velocity, fit a cubic polynomial to the 
velocity record (again leaving out the transit portion of the window when fitting), and remove the derivative of this polynomial from the acceleration record. Finally, this second-order corrected acceleration record was doubly integrated to compute the displacement record. No filtering was used.

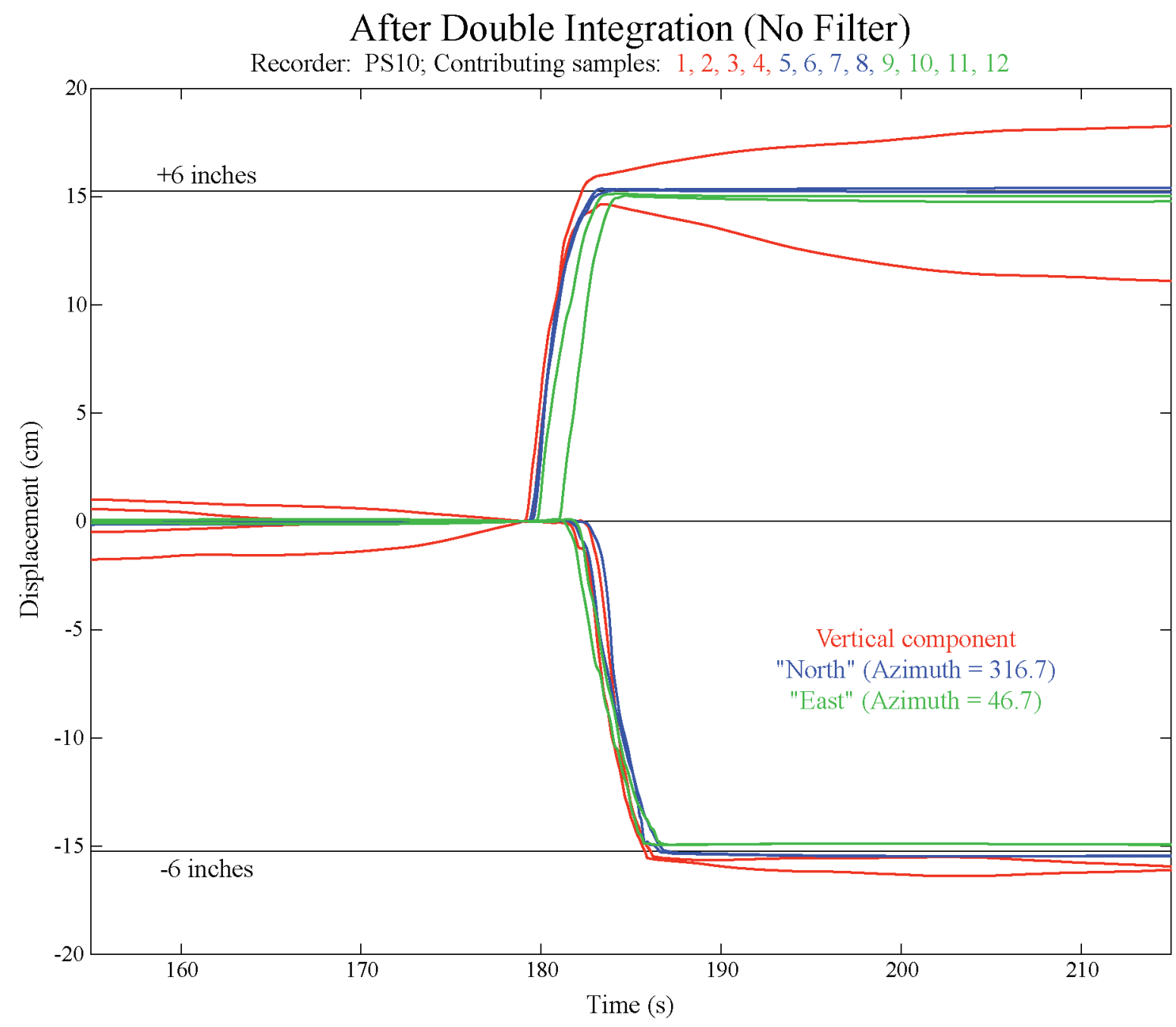

Figure 5 $\boldsymbol{b}$. Displacement traces as in Figure 5a, but for site PS10.

The results in Figures $5 a-c$ do not include either the effects of 16-bit quantization noise (at 200 samples per second) or of a small $0.5-\mathrm{Hz}$ random-telegraph signal introduced by the APSC recording software (a demeaning algorithm that ceases at the trigger time of the event but is present in the pre-event portion of the signal). Whether such distinctions are large or small, Figures $5 a-c$ more nearly reflect the optimal operational capabilities of the sensors, including very long 
pre- and post-event leads for baseline stabilization and the benefit of low, 24-bit quantization noise.

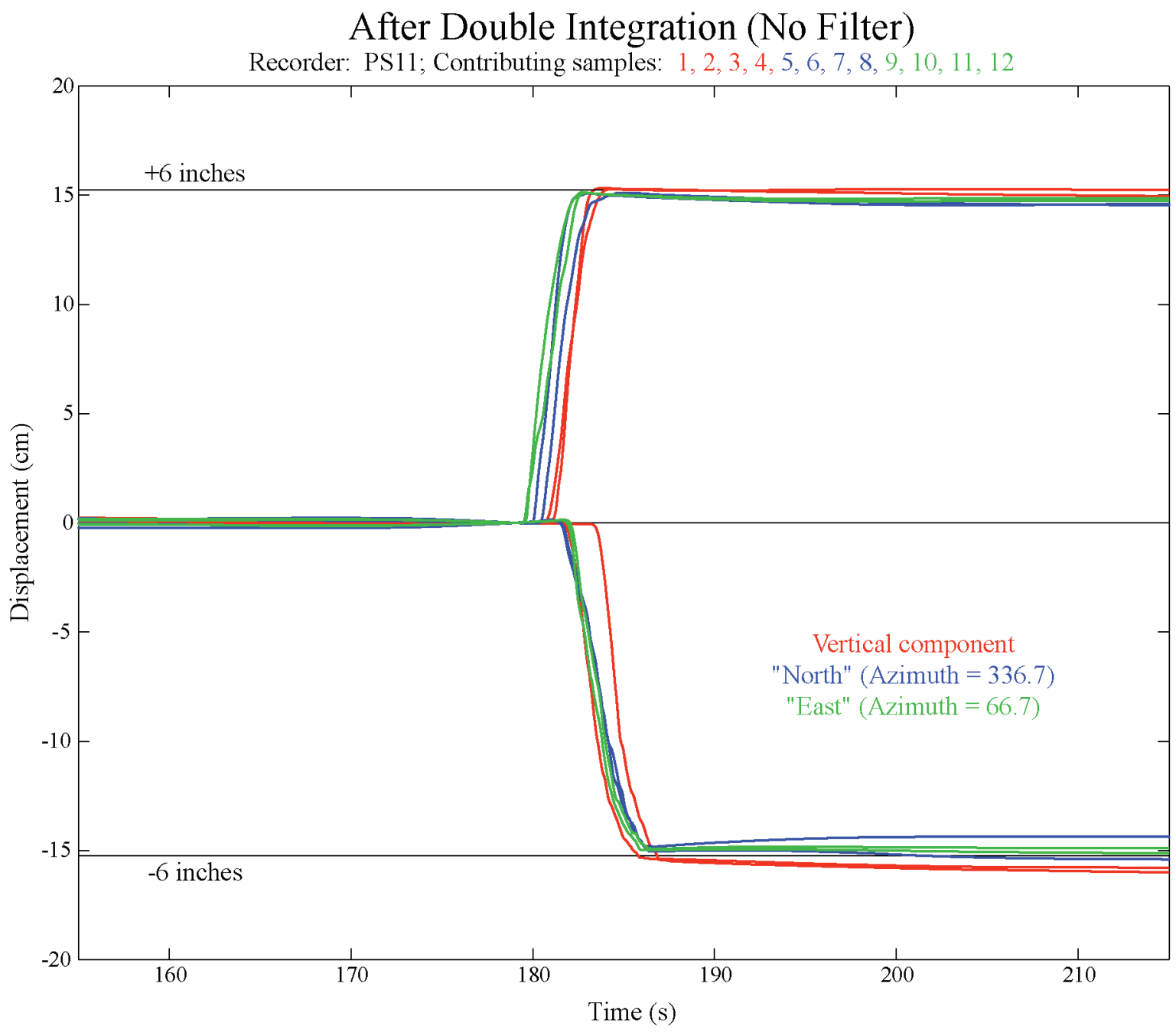

Figure 5c. Displacement traces as in Figure 5a, but for site PS11.

And those sensor capabilities are remarkable. After 25 years deployment in a hostile thermal environment, the baseline stability and amplitude precision of these nine accelerometers is as good as any we have seen. There is up to about $4-\mathrm{cm}$ baseline instability over the 60-s integration window for the two upward transitions of the vertical component of PS10. (During accelerometer calibrations, we also noted significantly higher self-noise on this component than on any other, and that the noise is asymmetric with respect to the applied acceleration signal. We suspect that this noise source may contribute to the double-integration drift issue and its asymmetric behavior for this one sensor.) The eight other accelerometers we tested were stable to within 
$1 \mathrm{~cm}$ over $60 \mathrm{~s}$ and recovered the $15.24-\mathrm{cm}$ step to within $2 \%$, if measured from just before to just after the transit interval. Figure 6 summarizes these results.

(In this Report, the final recalibration of the RefTek ${ }^{\mathrm{TM}}$ recorders was not applied to the double integration tests. Therefore, the values reported here are about $0.5 \%$ higher than they would be if properly corrected. If corrected, recovery of the step would average just over $99 \%$ of the true value rather than under $100 \%$ in Figure $6 \mathrm{~b}$, for example.)
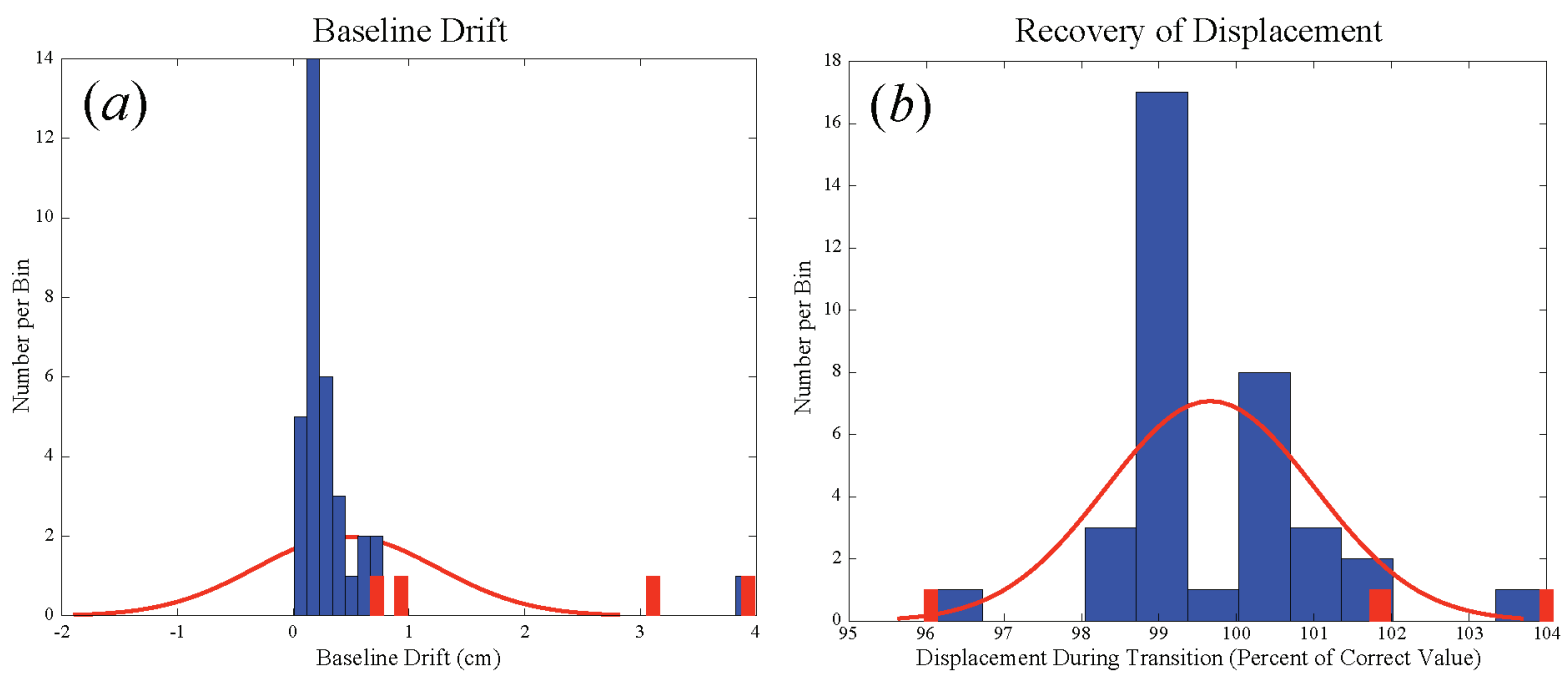

Figure 6. (a) Histogram of maximum baseline deviations for all components at all three Pump Stations, exclusive of the transition interval. Red bars highlight the four individual, unbinned values from PS10's Vertical component. (b) Histogram of the accuracy of displacement recovery during the transition intervals only, as a percentage of the correct value. PS10's Vertical is shown in red at higher precision. Bestfitting Gaussians are also shown.

\section{PROCESSING THE SEISMOGRAMS}

The original seismograms recorded by APSC are modified from raw signals according to a series of scale factors, principally for the gain of the accelerometer (including the $1.91 \mathrm{~K} \Omega 1 \%$ resistor in the associated amplifier), the gain of the filters, and the conversion scale factor (counts/V) of the analog-to-digital converter (ADC). Those stations with West-positive analog traces also are inverted to East-positive digital traces, including all three stations in this Report. No correction other than gain is made for the filters.

These scaling relationships, as applied to the seismograms originally released by APSC and the USGS in 2002, are given in Table 4 and shown schematically in Figure 2. The units of the recorded seismograms are g's, to a precision of $0.1 \mathrm{ng}$; however, the resolution of the ADC is 
about $45 \mu \mathrm{g}$. These seismograms were rescaled one more time into $\mathrm{cm} / \mathrm{s}^{2}$ (simply multiplied by the ICWM standard, $980.665 \mathrm{gal} / \mathrm{g}$ ), reformatted, and then released through the USGS Web site jointly by APSC and the USGS, originally in 2002 and in slightly modified form in 2003.

Table 4. Scaling factors used by APSC to process seismograms originally released by APSC and USGS in 2002 and 2003. The three right-hand columns are inferred from the values in the APSC "registry" and the APSC filter-gain factor $(2.51 \times)$. In this Table, "g" is the local value at each site.

\begin{tabular}{|c|c|c|c|c|c|c|c|}
\hline \multicolumn{8}{|c|}{ Gain Factors as Applied by APSC in November, 2002} \\
\hline \multirow{2}{*}{ Station } & \multirow{2}{*}{ Component } & \multicolumn{3}{|c|}{ From APSC Registry } & \multicolumn{3}{|c|}{ Interpreted Values } \\
\hline & & $\mathbf{m A} / \mathbf{g} \dagger$ & Counts/V & $\mathbf{V} / \mathbf{m A}^{*}$ & $\mathrm{~V} / \mathrm{g}(\mathrm{ADC}) \dot{\varphi}$ & $\mathrm{V} / \mathrm{g}$ (vault) $\stackrel{\oplus}{\dagger}$ & $\mu \mathrm{g} /$ Count \\
\hline \multirow{3}{*}{ PS09 } & Vertical & 1.502 & 3276.7 & 4.79 & 7.201 & 2.869 & 42.382 \\
\hline & North & 1.399 & 3276.7 & 4.79 & 6.707 & 2.672 & 45.503 \\
\hline & East & 1.334 & 3276.7 & 4.79 & 6.395 & 2.548 & 47.720 \\
\hline \multirow{3}{*}{ PS 10} & Vertical & 1.418 & 3276.7 & 4.79 & 6.798 & 2.708 & 44.893 \\
\hline & North & 1.377 & 3276.7 & 4.79 & 6.601 & 2.630 & 46.230 \\
\hline & East & 1.359 & 3276.7 & 4.79 & 6.515 & 2.596 & 46.842 \\
\hline \multirow{3}{*}{ PS11 } & Vertical & 1.388 & 3276.7 & 4.79 & 6.654 & 2.651 & 45.863 \\
\hline & North & 1.418 & 3276.7 & 4.79 & 6.798 & 2.708 & 44.893 \\
\hline & East & 1.301 & 3276.7 & 4.79 & 6.237 & 2.485 & 48.930 \\
\hline \multicolumn{8}{|c|}{ *Subsumes filter-gain factor $(2.51 \times)$ and $1.91 \mathrm{~K} \Omega$ scale factor. } \\
\hline & local $g$ at & & & & & & \\
\hline
\end{tabular}

This final scaling step by the USGS introduced two new small errors: scaling by a value of "g" not equal to local acceleration of gravity where the accelerometers were calibrated (at individual station vaults), and a small additional dose of quantization noise. The first and largest quantization noise is introduced when the signals are originally digitized, at a resolution of about $45 \mu \mathrm{g} /$ count. Since these are "one-shot" signals (an earthquake), rather than continuous signals ( such as noise), the quantization noise is approximately equal to a full digital count. (In contrast, quantization noise for stationary signals, such as stochastic noise or continuous sine waves, is about $1 / \sqrt{12} \approx 0.29$ of one digitizer count. Note these values are the quantization noise of a given sample. They are not measures of the whole record, for example of one's ability to measure a signal mean. Quantization noise is not a function of the length of the record - see any standard signal processing text.)

When scaled to $\mathrm{cm} / \mathrm{s}^{2}$ and reformatted in the USGS "SMC" format (which uses fivesignificant-digit floating point values), additional, variable quantization noise as large as $\sim 10 \mu \mathrm{g} /$ count also is added. (Thus, we do not use this format here.) The remaining quantization 
at $0.1 \mathrm{ng} /$ count when APSC formats the data contributes negligible noise. (We used the APSC record as the input to our own processing.)

Although the SMC-formatting noise is small, it would be best if the traces originally released in that format were no longer used or reprocessed, but in their stead the seismograms released here were used. The seismograms released here are processed with the full precision of the original APSC seismograms preserved (quantization noise about $45 \mu \mathrm{g} /$ count at the ADC and nothing added since then other than MatLab ${ }^{\text {TM }}$ IEEE double-precision floating point numerical noise). We release them in the COSMOS version 1.2 format, which allows us to scale the values without significant additional quantization noise. We may later release them also in the nascent COSMOS tagged format, which should be easier to read and use.

This section describes the process we use to convert from the original 2002 APSC seismograms (0.1-ng-resolution scaled and formatted versions of the $\sim 45 \mu \mathrm{g} /$ count original digitizer records) to the form(s) released with this 2005 Open-file Report.

\section{SIGNAL FLOW AND GOVERNING EQUATIONS}

Figure 2 summarizes the signal flow and examples of the values used at the time of the Denali fault earthquake to correct the seismograms (cyan-colored values), compared to equivalent values now available (magenta-colored values). We use the example of the "North" component of PS10, which recorded the largest accelerations from this event. The signal flow through the system is also expressed in Equations (1a), (2), and (3), where Equation (1a) subsumes the gain and sensitivity factors (with either the 2002 or our final calibration values from this report) illustrated in Figure 2 while Equations (2) and (3) are the high-pass and low-pass filters, expressed as Laplace transfer functions (voltage in, voltage out). The gain factors are

$$
a_{d i g}=C_{A D C} p G_{2}\left(R_{1.91 K} S_{m A}\right) a_{i n},
$$

where $a_{i n}$ is the ground motion in g's and $a_{d i g}$ is the recorded acceleration in counts, uncorrected for filter response other than filter gain. (Note that " $g$ ' $s$ " are, in both cases, according to the local value of gravitational acceleration at the accelerometer's calibration site, which varies by about $0.2 \%$ between the sites studies here, including at the vault, where APSC did its calibrations, and at Menlo Park, where our precision calibrations were made. We correct for this factor below, but be cautioned to use values expressed in $\mathrm{V} / \mathrm{cm} / \mathrm{s}^{2}$ [or the last row] in Tables $2 a-c$ when in need of 
absolute calibrations $-g$ is a variable.) $C_{A D C}$ is the ADC sensitivity in counts/V, $p$ is positive or negative unity for correcting polarity on some East-West components, $G_{2}$ is the gain of the filters at $2.0 \mathrm{~Hz}$, and $\left(R_{1.91 \mathrm{~K}} S_{\mathrm{mA}}\right)$ is sensitivity of the accelerometer and buffer amplifier system taken together, in V/g. $S_{\mathrm{mA}}$ is the accelerometer sensitivity in $\mathrm{mA} / \mathrm{g}$; these values were not measured for this Report. Since the output of the system also depends on the value of the resistor used (but not on the rest of the amplifier because its design obviates sensitivity to the values of other components), we tested the outputs of the amplifiers (that is, the values of $R_{1.91 \mathrm{~K}} S_{\mathrm{mA}}$ in $\mathrm{V} / \mathrm{g}$ ) and report and use these combined values exclusively.

During routine APSC processing, as was occurring on 03 November 2002, software performs the inverse computation to Equation ( $1 a$ ), using then-available values of calibration constants, and recording the estimated values of $a_{i n}$, which we call $a_{i n}^{\prime}$ :

$$
a_{\text {in }}^{\prime}=\left[C_{A D C} p G_{2}\left(R_{1.91 K} S_{m A}\right)\right]_{\text {estimated }}^{-1} a_{d i g}
$$

Referring to the names of poles and gains given in Tables $3 a-c$, the $S$-plane transfer function defining the high-pass filter is

$$
H_{H}(s)=\frac{G_{H} s^{2}}{\left(s-s_{H 1}\right)\left(s-s_{H 2}\right)}=\frac{G_{H} s^{2}}{\left[s^{2}-\left(s_{H 1}+s_{H 2}\right) s+s_{H 1} s_{H 2}\right]}
$$

and that defining the low pass filter is

$$
H_{L}(s)=\frac{G_{L}}{\left(s-s_{L 1}\right)\left(s-s_{L 2}\right)}=\frac{G_{L}}{\left[s^{2}-\left(s_{L 1}+s_{L 2}\right) s+s_{L 1} s_{L 2}\right]} .
$$

In the right-side of each equation, the coefficients are real. The 2.0-Hz gain term, $G_{2}$, in Equations $(1 a, b)$ is formally described (e.g., Wielandt, 2002) by

$$
G_{2}=\left|H_{H}(j \omega) H_{L}(j \omega)\right|_{2.0 H z},
$$

however, because the two filter corners are far apart and $2.0 \mathrm{~Hz}$ is well away from both, $G_{2}$ turns out to be only five parts per million different from the product $G_{H} G_{L}$ and does not bear special treatment. It is sufficient to approximate it as $G_{2} \approx G_{H} G_{L}$, therefore in the deconvolution of the high-pass filter there is no need to make any additional corrections for the gain factors not already expressed in Equations $(1 a, b)$, but only to deal with the poles and zeroes of Equations (2) and (3). 


\section{GAINS AND SENSITIVITIES}

Thus, there are just two calibration steps in converting from the seismograms recorded by APSC to those released with this Report. First we correct for changes to the sensitivities and gain factors in Equations $(1 a, b)$ to reflect current information and yielding "Volume 1" (i.e., original, uninterpreted) records (to use California Institute of Technology "Blue Book" terminology, and excepting that instrument-gain corrections also have been applied). Second, we deconvolve the high-pass filter from these "Volume 1" records and integrate the result, yielding broadband acceleration, velocity, and displacement records (equivalent to "Volume 2", i.e., interpretive, Blue Book records). Each step requires some explanation.

The first is discussed in this section and produces filtered but calibrated acceleration records that constitute the "Volume 1" data set - uninterpreted accelerograms. In most cases, these data are very similar to the acceleration records originally released, with gain changes less than $1.5 \%$, but in the case of PS11 there is a significant gain change of $\sim 9 \%$.

The procedure is as follows:

$$
a_{\text {gal }}=g_{\text {Menlo }} a_{\text {in }}=g_{\text {Menlo }} \Delta_{C} \Delta_{F} \Delta_{S} a_{\text {in }}^{\prime}
$$

where the $a_{i n}^{\prime}$, the original APSC seismograms (in local g's), is defined in Equation ( $\left.1 b\right)$. The various $\Delta$ 's are ratios of the old to new calibration values (not the other way around because of the inverse in Equation $(1 b)$ ), and are enumerated in Table 5 for all components. The term $g_{\text {Menlo }}$ is the local value of gravity where we precisely calibrated the accelerometers, Menlo Park, California, and scales the records from g's to $\mathrm{cm} / \mathrm{s}^{2}$, hence " $a_{g a l}$ ".

In addition, there is the complication for $\mathrm{D}_{\mathrm{S}}$, the sensor correction factor, of the local value of "g" at the site of each calibration measurement. These calibration sites were the individual Pump Stations for the APSC values but Menlo Park, California, for the USGS values. The Alaskan values must therefore be shifted to the Menlo Park value as

$$
\Delta_{S}=\frac{\left(R_{1.91 K} S_{m A}\right)_{A P S C}}{\left(R_{1.91 K} S_{m A}\right)_{U S G S}} \frac{g_{\text {Menlo }}}{g_{P S x x}} .
$$

The values of "g" at Menlo Park, PS09, PS10, and PS11 are 979.945 $\pm 0.002,982.054 \pm 0.004$, $981.927 \pm 0.002$, and $981.906 \pm 0.004 \mathrm{~cm} / \mathrm{s}^{2}$, as obtained from the National Geodetic Survey's 
"Surface Gravity Prediction" model (http://geodesy.noaa.gov/TOOLS/Gravity/gravcon.html). The net result of Equations (5) and (6) (right-hand column of Table 5) is $\leq 1.5 \%$ change in amplitudes at PS09 and PS10. However, largely because of the significant change in accelerometer sensitivities at PS11, those three components decrease in amplitude by about $9 \%$.

The next steps in processing the acceleration records, deconvolution of filter responses and integration to velocity and displacement, involve a degree of subjective judgment on the part of the analyst. This is particularly so for PS10 because we can expect near-field terms - the reader is cautioned at the outset to use this site's records thoughtfully. Specifically, we provide two versions of the interpreted ("Volume 2") records for PS10. The first ("Option 1") is equivalent to the acausal processing for sites PS09 and PS11 and is designed for users interested in dynamic modeling of structures, response spectra, and other matters in which the phase angles of the records must be kept coherent at all frequencies (Boore and Akkar, 2003).

Table 5. Ratios between original APSC and the calibration factors of this Report - the D factors, cf. Equation (5).

\begin{tabular}{|c|c|c|c|c|c|}
\hline \multirow[t]{2}{*}{ Station } & \multirow[t]{2}{*}{ Component } & \multirow{2}{*}{$\begin{array}{l}\text { Accelerometer } \\
\Delta_{\mathrm{S}}, \text { Equation (6) }\end{array}$} & $\begin{array}{l}\text { Filter Gain } \\
\left(\mathbf{G}_{2}\right)_{\mathrm{APSC}}\end{array}$ & $\begin{array}{l}\text { ADC Sensitivity } \\
\left(\mathrm{C}_{\mathrm{ADC}}\right)_{\mathrm{APSC}}\end{array}$ & \multirow{2}{*}{$\begin{array}{l}\text { Net Correction } \\
\frac{\text { APSC }}{\text { USGS }}\end{array}$} \\
\hline & & & $=\overline{\left(\mathrm{G}_{2}\right)_{\mathrm{USGS}}}$ & $\Delta_{\mathrm{C}}=\frac{\left(\mathrm{C}_{\mathrm{ADC}}\right)_{\mathrm{USGS}}}{}$ & \\
\hline \multirow{3}{*}{ PS09 } & Vertical & 1.00423 & 0.9837 & 0.999985 & 0.98782 \\
\hline & North & 1.00297 & 0.9869 & 0.999985 & 0.98980 \\
\hline & East & 1.00410 & 0.9873 & 0.999985 & 0.99131 \\
\hline \multirow{3}{*}{ PS10 } & Vertical & 1.00257 & 0.9886 & 0.999985 & 0.99110 \\
\hline & North & 1.00244 & 0.9877 & 0.999985 & 0.99005 \\
\hline & East & 1.00049 & 0.9847 & 0.999985 & 0.98519 \\
\hline \multirow{3}{*}{ PS11 } & Vertical & 0.92546 & 0.9847 & 0.999985 & 0.91129 \\
\hline & North & 0.92691 & 0.9859 & 0.999985 & 0.91380 \\
\hline & East & 0.91827 & 0.9870 & 0.999985 & 0.90628 \\
\hline
\end{tabular}

\section{DECONVOLUTION AND DOUBLE INTEGRATION OF THE SEISMOGRAMS}

In contrast, "Option 2" is unique to PS10 records and is offered for those needing to preserve the total displacement of the ground, for example in rupture modeling, testing the responses of tall structures to interfering start and stop waves, and so forth. "Option 2" is causal processing broadly similar to that of Ellsworth et al. (2004). Such causal processing was not performed for PS09 or PS11 because, as discussed below, the resolution of the digitizers does not appear to have been quite sufficient to recover total ground displacements at those locations. 
Our processing steps for all stations are summarized in Tables $6 a, b$. The first step (gain corrections) was discussed above.

As a preliminary step to both causal and acausal processing, we found that making a simple correction for the baseline of the "Volume 1" records was helpful. We first removed the mean of the trace up to the point of the earliest $P$-wave motion, yielding displacement records with large, roughly quadratic departures from zero (Figures $7 a-c$ ). Since these deviations appear to be tangent to the baseline where the departures begin, we model them as tangent quadratic functions in displacement, corresponding to simple steps in acceleration (that is, without any Dirac-delta singlets or their derivative, infinite-amplitude Dirac doublets, deriving from the first- or secondorder differentiation of constants, respectively).

(a) PS09 Demeaned "Volume 1" Acceleration,

Doubly Integrated and fit by Tangent Quadratics

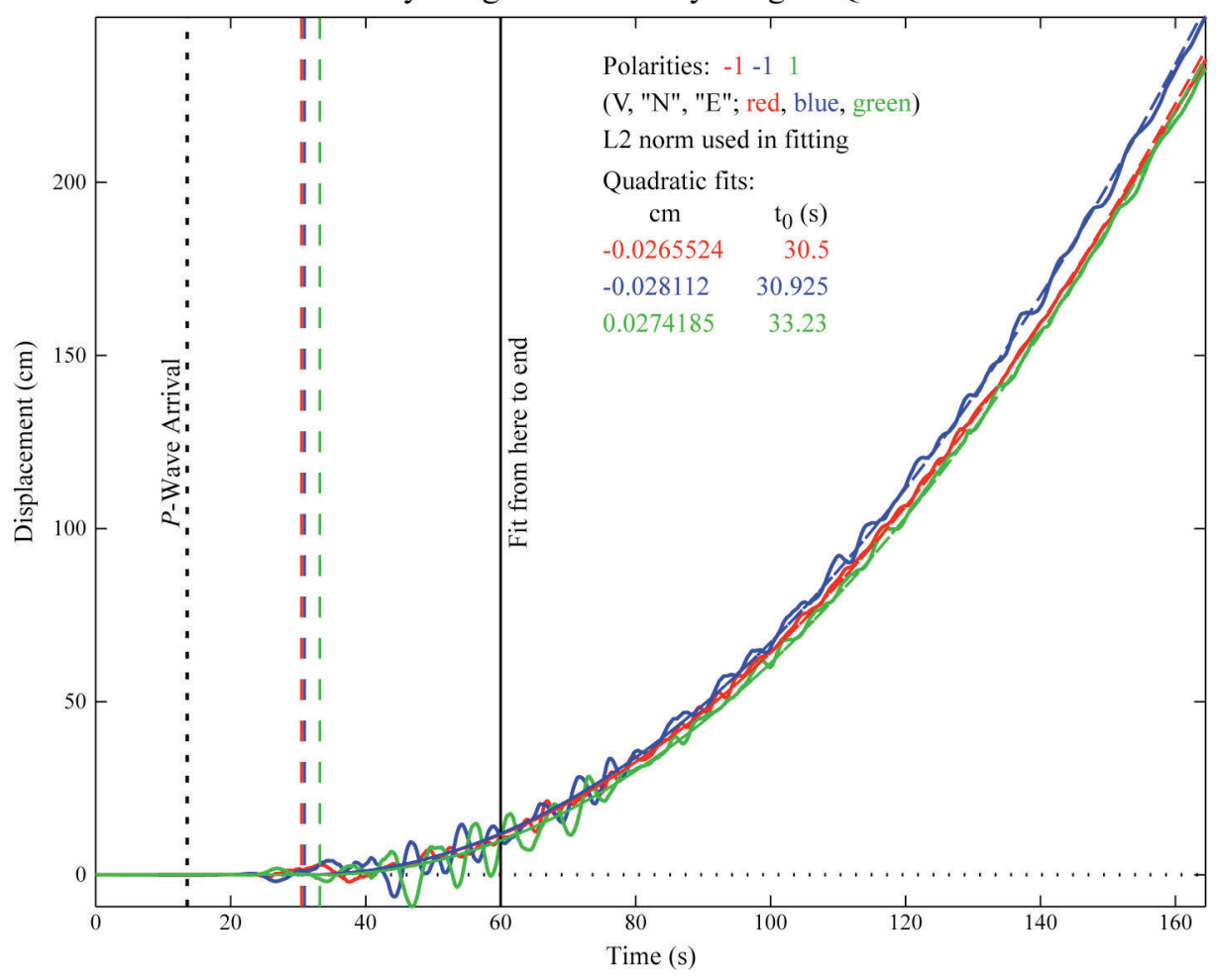

Figure 7. The "Volume 1" acceleration records integrated to displacement. Curved dashed lines show the least-squares ("L2") fits of tangent quadratic functions fitted to the portions from the vertical solid line to the ends of the traces. Vertical dashed lines indicate the tangent points of these fitted lines to zero displacement. Colors indicate components. Polarities have been reversed as indicated to make the deviations all positive for computation and comparison. (a) PS09; (b) PS10; and (c) PS11. 


\section{Demeaning and Removal of Acceleration Step}

At each station, individual components begin these quadratic deviations at roughly the same time (indeed, they can be modeled almost as well with synchronicity imposed) but they are not synchronous between stations. They are instead coincident with the large $S$-wave/surface-wave motions at each site. At PS09 and PS10, the amplitudes of these displacement quadratics are about equal between all three components, while at PS11 the "East" component deviates about 2.5 times as far as the others. Nevertheless, the large size of the vertical-component deviations at all sites and the apparent synchronicity of these baseline acceleration steps suggest either an electronic source or a surprisingly systematic mechanical source. (The latter, a synchronous mechanical cause presumably triggered by large $S$-wave motions, was not revealed or hinted at by any sensor/amplifier test that we performed. Furthermore, the design of the Sundstrand ${ }^{\mathrm{TM}}$ accelerometers is unlikely to create such a step since it is a monolithic laser-cut quartz mass and spring, lacking parts that are likely to suffer "oil canning” or slip anomalies.) While the variation in polarities would be unexpected from an electrical source, that source nevertheless remains a possible explanation, perhaps in power supplies or voltage references in response to variations, during strong shaking, in the power supplied to the plant.

Alternately (Figures $7 d, e$ ), there may be some contribution from a slight asymmetry evident in the background noise, perhaps caused by ADC imperfections. The noise measured prior to the arrival of the $P$ wave, even when demeaned before histogram binning, when best fit by a Gaussian, shows a slight offset of the mean, an asymmetry. This offset can account for about $37 \mathrm{~cm}$ of displacement after $160 \mathrm{~s}$ if the "step" is applied at $0 \mathrm{~s}$. That is, it is of the same magnitude as the observed quadratic departures. However, this pattern does not readily explain why the quadratic departures in Figures $7 a-c$ begin at about the time of the $S$ wave. In the end, we must accept that the cause of the apparent acceleration steps near the $S$ wave is as yet unidentified.

Whatever the cause of these acceleration steps, removal at this early stage of processing is largely successful and helpful in reducing the size and complexity of baseline-error signals that have to be dealt with at later processing stages. Nevertheless, after this first correction step, PS09 retains a moderately large signal in displacement that appears to be an exponentially growing long-period sine wave (not readily visible in Figure $7 a$, but obvious in the residuals to that fit, not shown here). It's source may well be electrical since it appears on all three channels with 
comparable magnitude, though varying phase. Subsequent filtering removes it successfully since its frequency is well below the pass-band of interest.

Table 6a. Enumeration of accelerogram processing steps required to produce both "Volume 1" and interpretive, "Volume 2" records. PS09 and PS11 are covered here. The time $t_{0}$ is the beginning of the original (unpadded) trace, and $t_{P}$ is the arrival time of the $P$-wave. The notations " $[-1-1 \quad 1]$ ", etc. gives the polarities of the quadratic signals in Step 3 for the vertical, "North", and "East" components. The same order of components applies to the notations "[ 4.354 .485 .17 ] s", etc.

\begin{tabular}{|c|c|}
\hline $\begin{array}{c}\text { PS09 } \\
\text { (Acausal) }\end{array}$ & $\begin{array}{c}\text { PS11 } \\
\text { (Acausal) }\end{array}$ \\
\hline \multicolumn{2}{|c|}{ Correct gains (eqn. (5)) } \\
\hline \multicolumn{2}{|c|}{ SAVE gain-corrected ("Volume 1") records } \\
\hline Demean using $t_{0}$ to $t_{P}(13.550 \mathrm{~s})$ & Demean using $t_{0}$ to $t_{P}(14.860 \mathrm{~s})$ \\
\hline $\begin{array}{l}\text { Remove tangent quadratic in displacement } \\
\text { fit (L2) from } t=60 \mathrm{~s} \text { to end }(164.470 \mathrm{~s}) \\
\text { polarities }[-1-11]\end{array}$ & $\begin{array}{l}\text { Remove tangent quadratic in displacement } \\
\text { fit (L2) from } t=93 \mathrm{~s} \text { to end }(164.785 \mathrm{~s}), \\
\text { polarities [ } 1-1-1] .\end{array}$ \\
\hline $\begin{array}{l}\text { Pad with zeros front }(61.570 \mathrm{~s}) \text { and back } \\
\text { (including } 6 \text { extra samples to aid FFT } \\
\text { factorization, } 61.600 \mathrm{~s} \text { ); cosine taper } \\
\text { front } 5 \mathrm{~s} \text {, rear [ } 4.354 .485 .17 \text { ] s }\end{array}$ & $\begin{array}{l}\text { Pad with zeros front }(61.690 \mathrm{~s}) \text { and back } \\
\text { (including } 12 \text { extra samples to aid FFT } \\
\text { factorization, } 61.750 \mathrm{~s}) \text {; cosine taper } \\
\text { front } 5 \mathrm{~s} \text {, rear [ } 5.465 .415 .49] \mathrm{s}\end{array}$ \\
\hline \multicolumn{2}{|c|}{ Filter with acausal Butterworth: $3^{\text {rd }}$-order, 27.4 -s, forward and backward } \\
\hline \multicolumn{2}{|c|}{ Deconvolve, raising only DC $(0-\mathrm{Hz})$ bin of denominator above its original value (zero) } \\
\hline \multicolumn{2}{|c|}{ Demean from beginning of pad to $t_{P}$} \\
\hline \multicolumn{2}{|c|}{ Integrate by trapezoidal rule from acceleration to velocity to displacement } \\
\hline \multicolumn{2}{|c|}{ SAVE "Interpreted" ("Volume 2") Records } \\
\hline
\end{tabular}

\section{Signal and Noise}

In what follows, there is an underlying supposition of good signal present above some longperiod threshold and noise predominating below that frequency. This supposition is supported by Figures $8 a-c$, which show Discrete Fourier Transform (DFT) amplitude spectra for the nine "Volume 1" traces, that is, as corrected only for gains. Much of what follows is aimed at separating these two components and eliminating the noise. Using Figures $8 a-c$, we selected thresholds between signal and noise near periods of $19.3 \mathrm{~s}$ for PS09, $26.3 \mathrm{~s}$ for PS10, and $19.4 \mathrm{~s}$ for PS11. Our choices for filtering or its equivalent (i.e., modifications to the denominator during deconvolution) are guided by these threshold periods.

\section{Padding and Tapering}

In all cases the next step is to apply a cosine taper to a modest portion of each end of each trace, tapering $5 \mathrm{~s}$ of the pre-event portion and from 2.3 to $5.49 \mathrm{~s}$ of the final coda of each trace, 
as indicated in Tables $6 a, b$. These latter amounts were selected empirically to reduce tail-end anomalies in later processing steps. In some cases it was found that beginning the taper at peaks, troughs, or zero crossings of the long-period portion of the signal produced a good result. The sensitivity of the result to this rear-end tapering and its failure to correlate simply with signal characteristics is unexpected and, so far, unexplained.

Table $6 \boldsymbol{b}$. Like Table $6 a$ but for PS10, showing both versions of the processing.

\begin{tabular}{|c|c|c|}
\hline \multirow{2}{*}{$\begin{array}{l}\text { Station } \\
\text { Option }\end{array}$} & \multicolumn{2}{|c|}{ PS10 } \\
\hline & Option 1 (Acausal) & Option 2 (Causal) \\
\hline Step 1 & \multicolumn{2}{|c|}{ Correct gains (eqn. (5)) } \\
\hline (Save) & \multicolumn{2}{|c|}{ SAVE gain-corrected ("Volume 1") records } \\
\hline Step 2 & \multicolumn{2}{|c|}{ Demean using $t_{0}$ to $t_{P}(8.400 \mathrm{~s})$} \\
\hline Step 3 & \multicolumn{2}{|c|}{$\begin{array}{l}\text { Remove tangent quadratic in displacement fit (L2) from } t=44 \mathrm{~s} \text { to the end }(92.090 \mathrm{~s}) \\
\text { polarities }\left[\begin{array}{lll}1-1 & 1\end{array}\right]\end{array}$} \\
\hline Step 4 & $\begin{array}{l}\text { Pad with zeros front }(83.720 \mathrm{~s}) \text { and back } \\
\text { (including } 14 \text { extra samples to aid FFT } \\
\text { factorization, } 83.790) \text {; cosine taper } \\
\text { front } 5 \mathrm{~s} \text {, rear [ } 2.355 \text { ] s }\end{array}$ & $\begin{array}{l}\text { Pad with zeros front }(60.000 \mathrm{~s}) \text { and back } \\
\text { (including } 14 \text { extra samples for FFT } \\
\text { factorization, 60.070); cosine taper } \\
\text { front } 5 \mathrm{~s} \text {, rear [ } 2.355 \text { ] s }\end{array}$ \\
\hline Step 5 & $\begin{array}{l}\text { Filter with acausal Butterworth: 3rd-order, } \\
37.2 \text {-s corner, applied forward and backward }\end{array}$ & $\begin{array}{c}\text { Deconvolve, raising all bins of denominator } \\
\text { centered }>26.5 \text {-s (edge } 25.0 \mathrm{~s} \text { ) above original } \\
\text { values to mute long-period noise }\end{array}$ \\
\hline Step 6 & $\begin{array}{l}\text { Deconvolve, raising only } \mathrm{DC}(0-\mathrm{Hz}) \text { bin of } \\
\text { denominator above its original value (zero) }\end{array}$ & $\begin{array}{c}\text { Demean, using interval from } \\
\text { beginning of pad to } t_{P}\end{array}$ \\
\hline Step 7 & $\begin{array}{l}\text { Demean, using interval from } \\
\text { beginning of pad to } t_{P}\end{array}$ & $\begin{array}{c}\text { Fit (L2) and remove } 30 \text { th-order polynomial } \\
\text { to all but strong } S \text {-wave, targeting acausal } \\
\text { filter-error bounce }\end{array}$ \\
\hline Step 8 & \multicolumn{2}{|c|}{ Integrate by trapezoidal rule from acceleration to velocity to displacement } \\
\hline (Save) & \multicolumn{2}{|c|}{ SAVE interpreted ("Volume 2") records } \\
\hline
\end{tabular}

Next, substantial intervals of zeros were added as padding, (nearly) symmetrically to the front and rear of each trace, anticipating long-period, acausal filter processes. These acausal filters were either explicitly applied or came about because deconvolution can include an acausal, long-period component as a result of disabling an interval of long-period bins. The pads are retained throughout the remainder of the processing, essential to the validity and success of the process, and part of the resulting seismograms (Boore, 2005), though not plotted in most figures. 
(b) PS10 Demeaned "Volume 1" Acceleration,

Doubly Integrated and fit by Tangent Quadratics

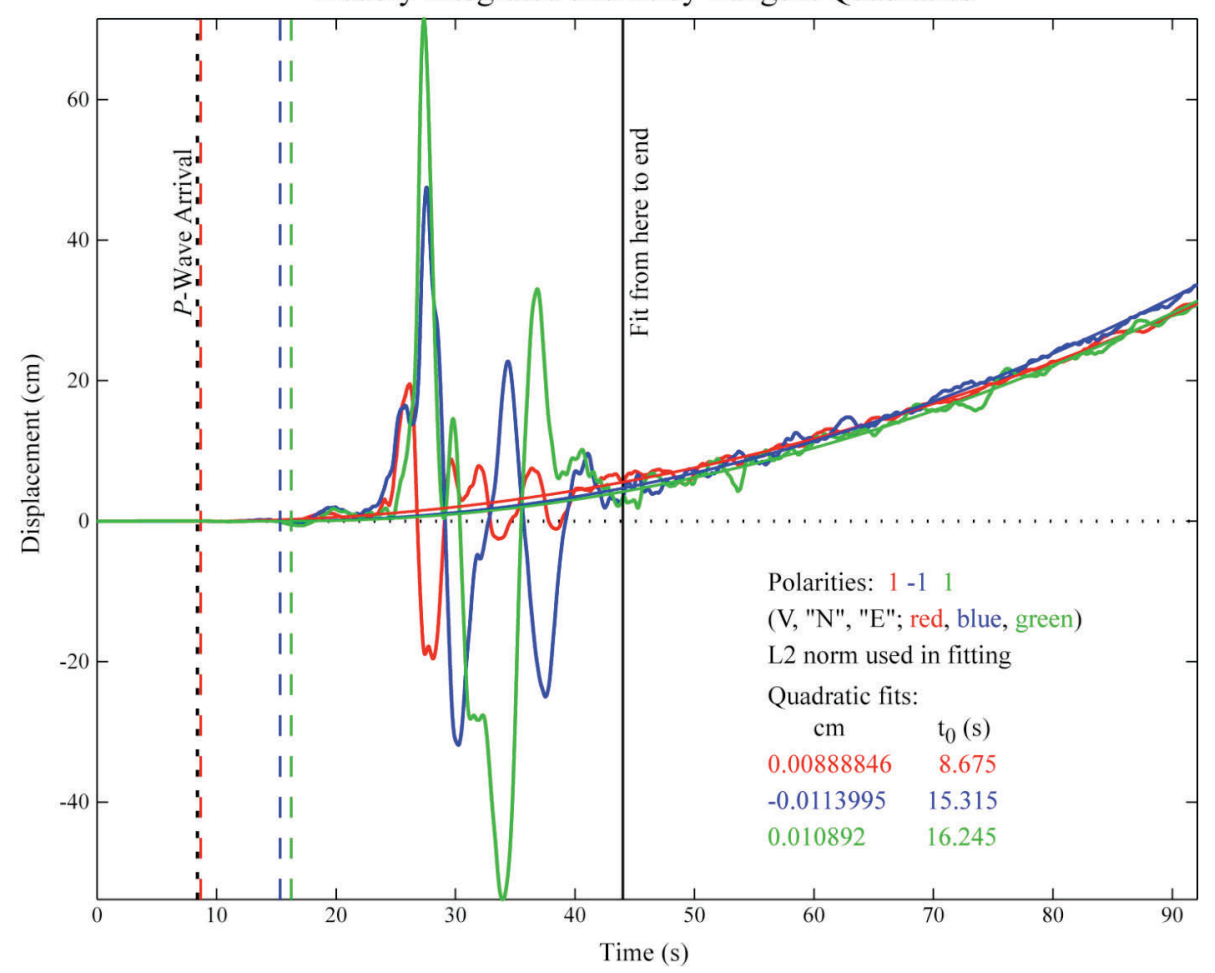

(c) PS11 Demeaned "Volume 1" Acceleration,

Doubly Integrated and fit by Tangent Quadratics

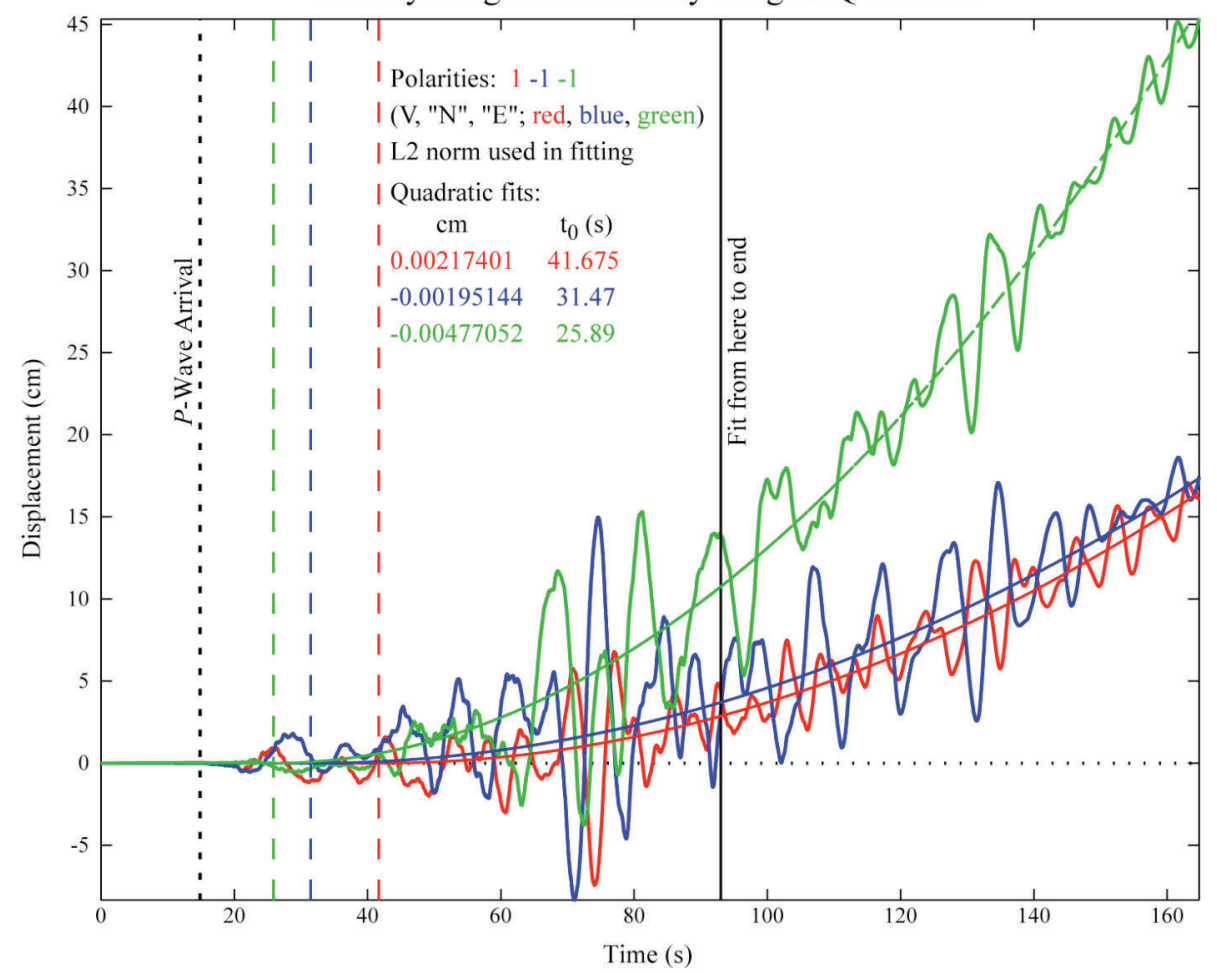

Figure 7. (cont.) 
(d) Pre-Event Noise Distribution; By Component

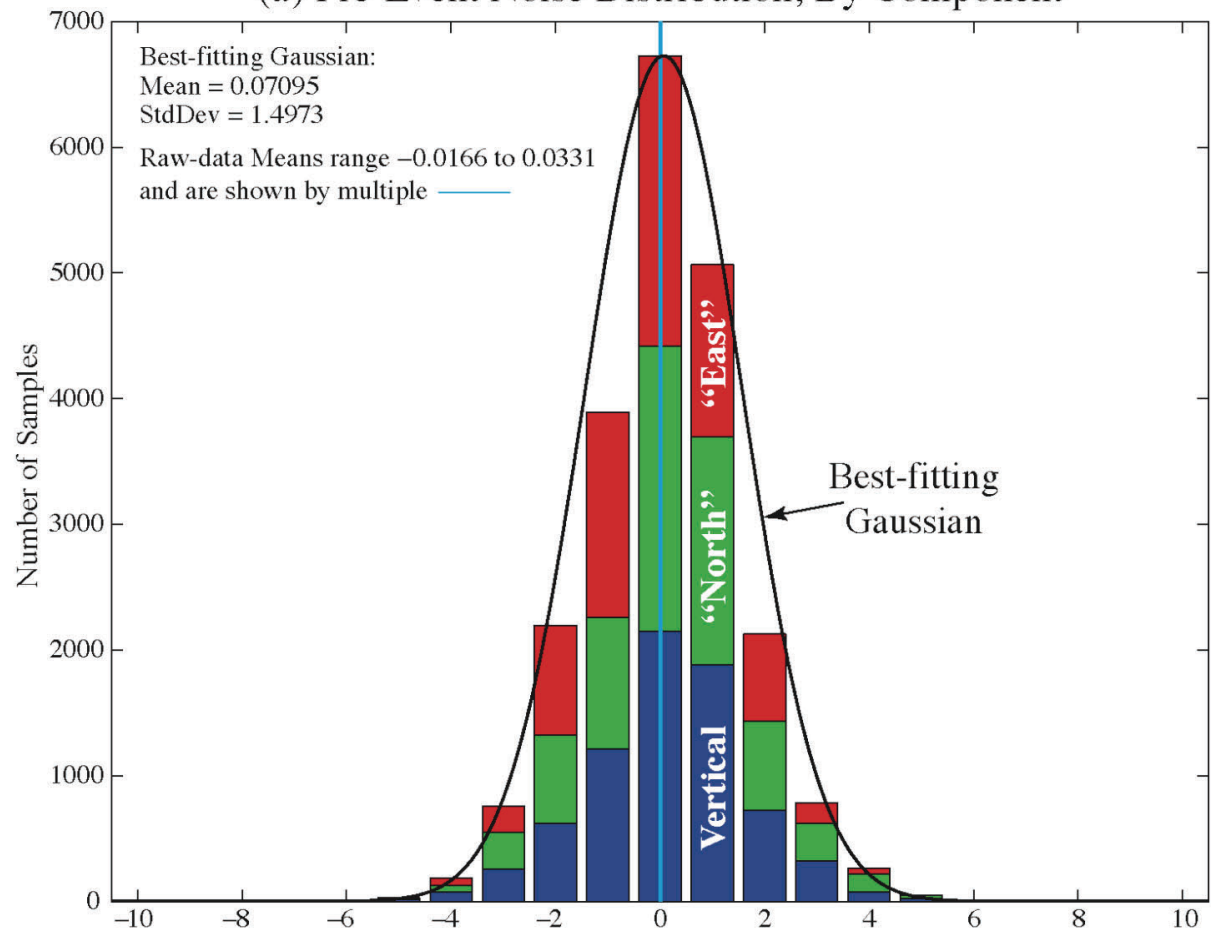

(e) Pre-Event Noise Distribution; By Site

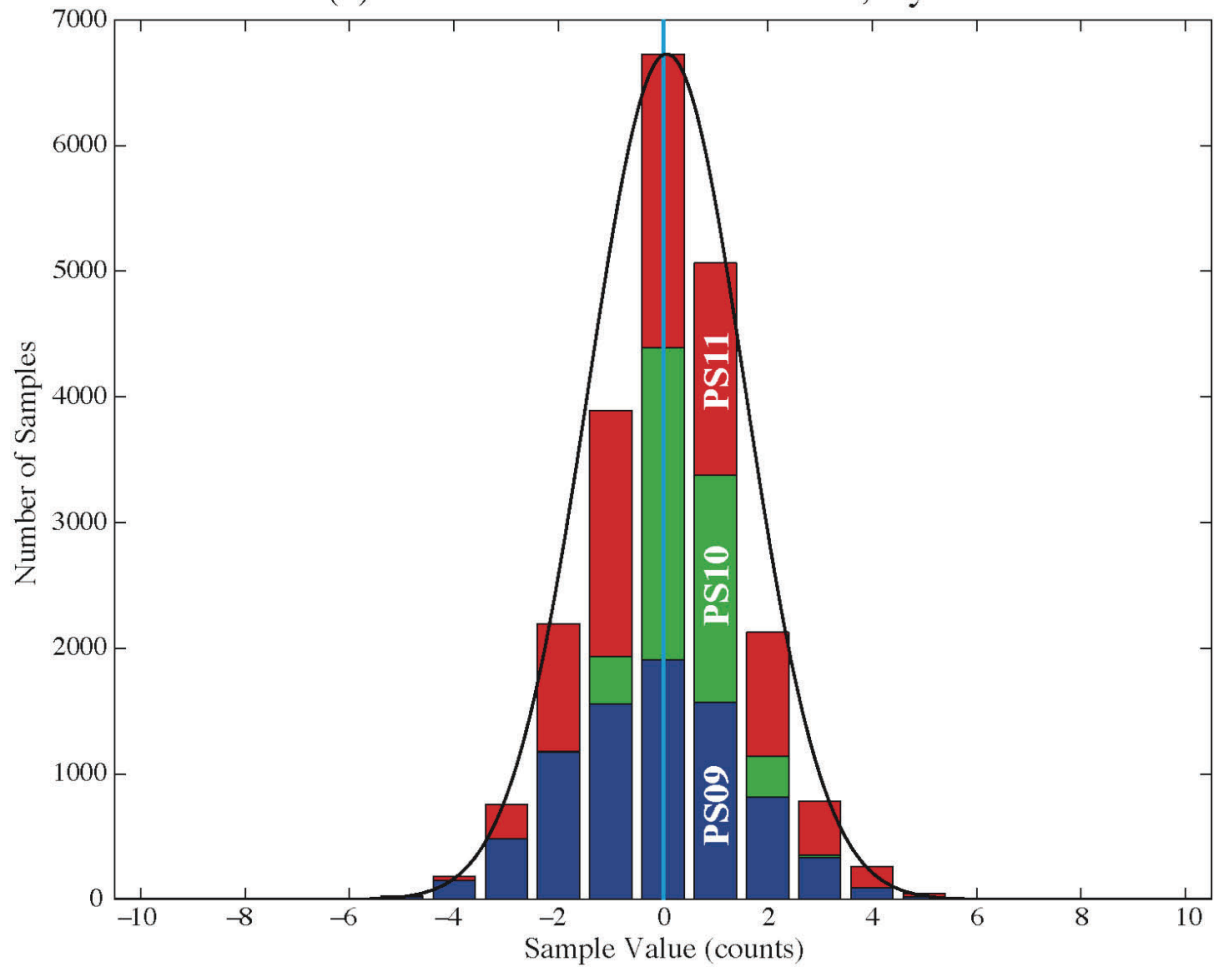

Figure 7. (cont.) Distribution of pre-event noise samples after noise-segment demeaning. Note slight offset between Gaussian mean and the zero-valued sample. Subdivided by component $(d)$ or by station (e). Cyan vertical lines show the values of noise-segment means removed prior to histogram binning, and are smaller than the offset of the fitted Gaussian by a factor of two. 
(a) "Volume 1" Individual and Ensemble-Averaged Amplitude Spectra, PS09

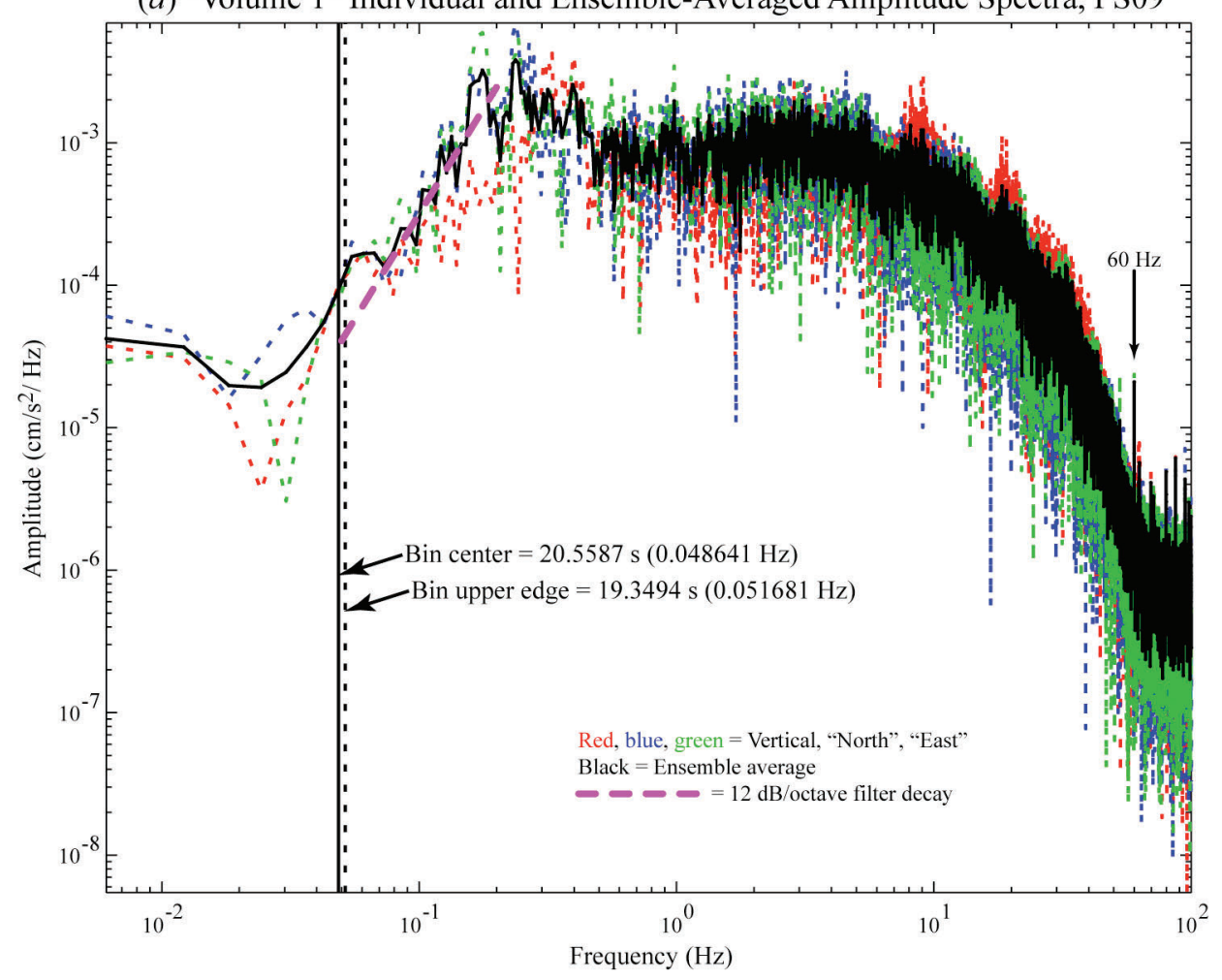

Figure 8. DFT amplitude spectra of the "Volume 1" (unpadded) acceleration records. Vertical solid and dashed lines show the periods (frequencies) we have chosen to control subsequent processing. Below these frequencies, the spectra deviate from the expected system frequency decay rate (dashed magenta line) indicating noise in the record. (Spectra are validly averaged [black spectrum] because signal-tonoise ratios will be about the same on all components and have nearly the same filter decay rates, 12 db/octave.) (a) PS09; (b) PS10; and (c) PS11.

Users are alerted to this final point. Filtering and integration are both performed over the entire, padded trace in order to account properly for the long tails of these filters without contaminating the seismograms per se. Failing to include the pads when integrating will not produce the same result as reported here nor a sensible result. The need for and validity of this technique has been described by Boore (2005) and applies even to the "causal" case of PS10's "Option 2" because of the acausal nature of the deconvolution "filter error" fraction, as described below.

The lengths of the zero pads are chosen to be at least as long as suggested by Converse and Brady (1992), $T_{z p a d}=1.5 n / f_{c}$, where $T_{z p a d}$ is the total length of zero padding in seconds, $n$ is the order of the Butterworth filter, and $f_{c}$ is the filter corner frequency in Hz. Half the pad is placed in front of the trace and half at the rear, protecting both ends of the trace from filter wrap-around effects and providing both ends of the trace with room for the filter transients to die away. Fur- 
thermore, note that the long front pad allows for a more accurate estimate of the mean when estimating an initial acceleration value.

(b) "Volume 1" Individual and Ensemble-Averaged Amplitude Spectra, PS10

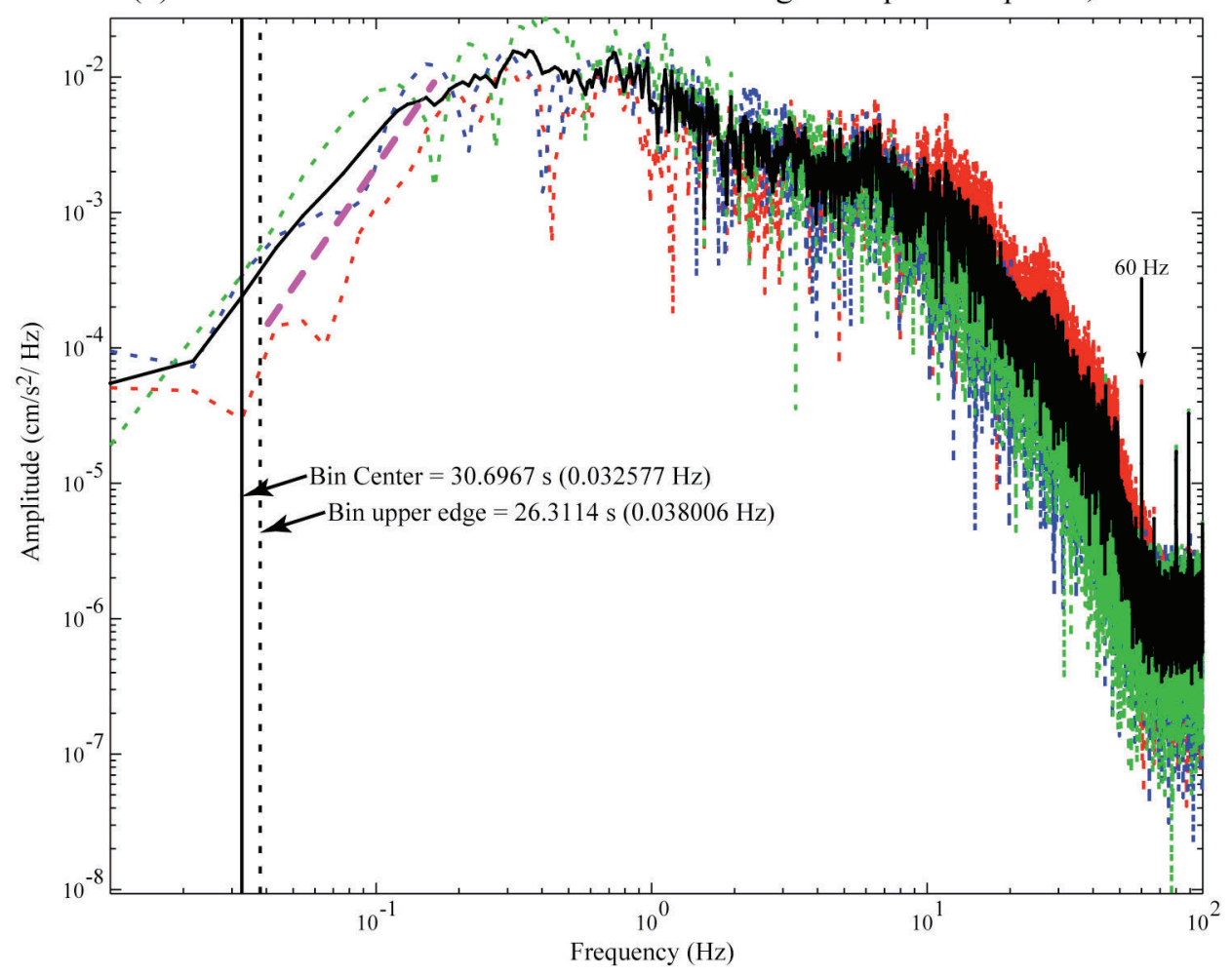

Figure 8. (cont.)

It should also be mentioned that a few additional zeros are added at the rear pad to make the total length of the trace factor into smaller primes for the purposes of FFTs. (In MatLab and most other FFTs it is no longer necessary to pad to a power of two - indeed, we have observed an FFT of 65537 points, a prime, execute in seconds.) We pad just enough that the largest prime factor is smaller than 100 to obtain fast FFT execution on very large traces. This process adds only a few additional zeros to the rear pad (Tables $6 a, b$ ).

\section{Butterworth "Error Filter" and PS10 Causal Processing}

There is the one more constraint on the minimum length of zero padding. We perform a deconvolution to mitigate the effects of the causal electronic 12 -s-corner Butterworth filters originally used at the instrument to high-pass filter the accelerograms. We use simple spectral division of the signal DFT by the Butterworth DFT. An example of a Butterworth DFT is shown in Figure 9. Following the method of Ellsworth et al. (2004) we use the filter poles and zeroes 
from Tables $3 a-c$ directly to generate time-domain impulse responses of individual filters, then use these responses for the spectral division.

(c) "Volume 1" Individual and Ensemble-Averaged Amplitude Spectra, PS11

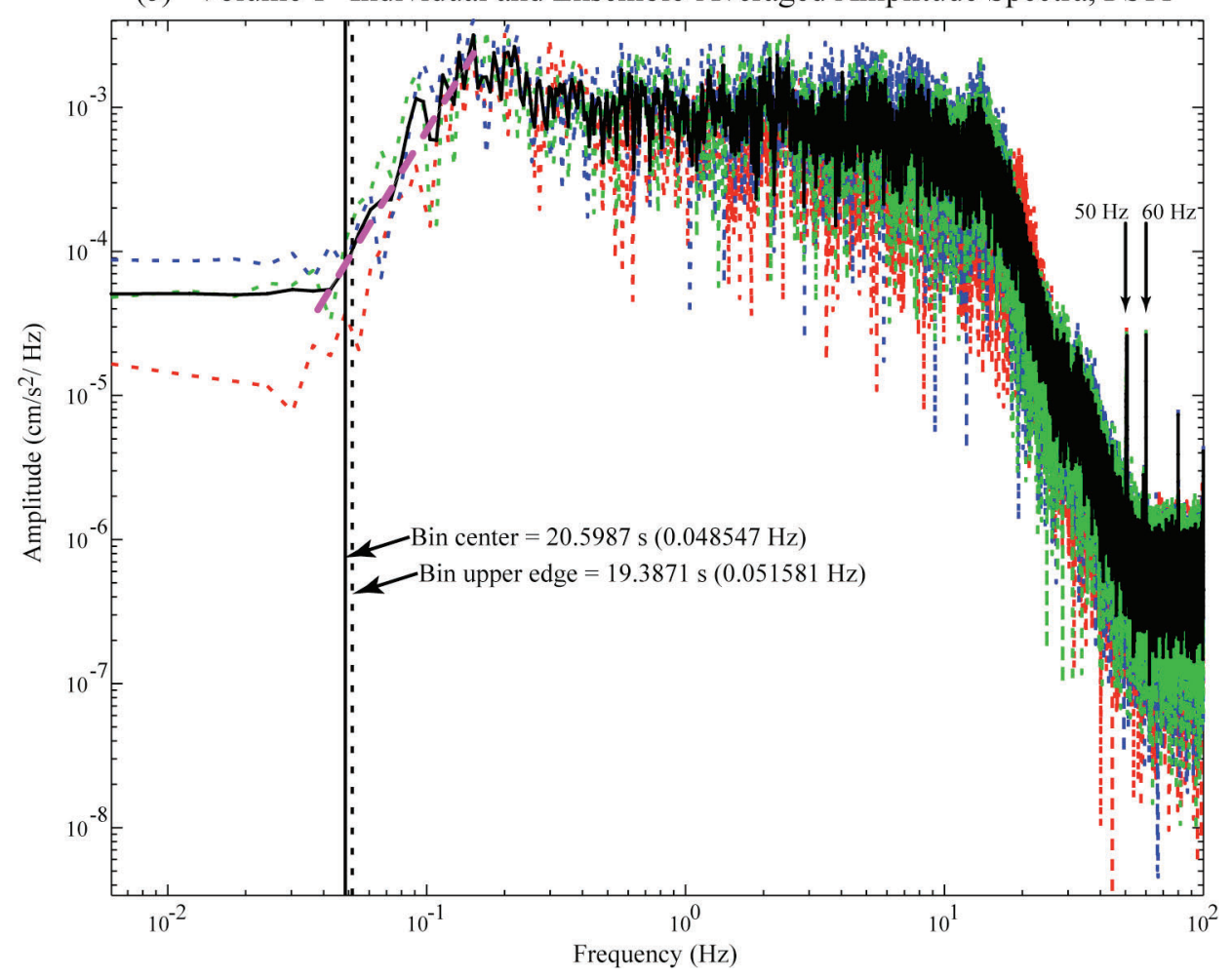

Figure 8. (cont.)

Ideally, therefore, the deconvolution step should be causal since the filters are causal. However, one must impose a constraint on the denominator, at a minimum to avoid dividing by zero (the "DC", 0-Hz, bin is always zero). And in one case (PS10, "Option 2", when we wish to preserve permanent ground displacement), we must also raise the values of many higher-frequency bins that would otherwise amplify noise in the original signals - we must raise the values of the denominator from $0 \mathrm{~Hz}$ all the way to the threshold period defined in Figure 8b, $26.3 \mathrm{~s}$. (In acausal processing of all stations, we instead use an acausal Butterworth filter prior to deconvolution to suppress these long-period noise signals so that we do not need to suppress them during deconvolution.) Thus in the causal processing of PS10, when the input signal (the numerator) contains noise at long periods, we must raise the values of the Butterworth filter's amplitude spectrum (the denominator in the deconvolution, as shown by the higher line in Figure 9). This change in the (frequency domain) amplitude response of the filter results in a significant differ- 
ence between the (time domain) impulse response of the modified Butterworth filter and the original, causal electronic filter. This difference in impulse responses between the true and modified electronic Butterworth filters, the latter being used for deconvolution, can be thought of as an acausal "error filter" (Figure 10). When this error filter is applied to the input, the groundmotion signal, it creates an acausal error output signal.

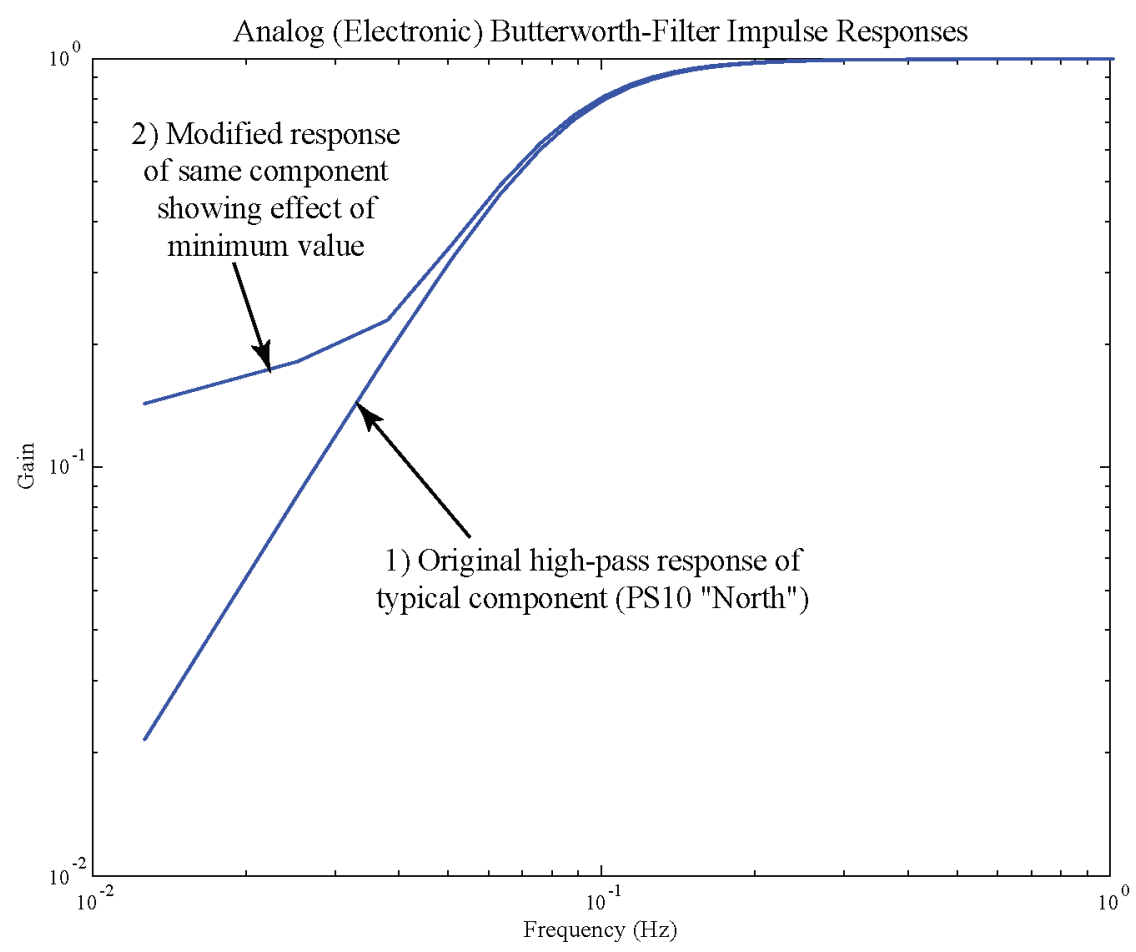

Figure 9. DFT amplitude spectra for one of the Butterworth low-cut filters which are to be deconvolved from the system response. The "North" channel for PS10 is shown, the others being similar. For comparison, the same channel has been raised to the level required for "Option 2" to prevent the amplification of long-period noise during deconvolution of this filter.

We therefore attempt to model, fit, and remove this acausal error output signal (as it appears in the velocity traces) in order to leave behind the true, causal ground-motion signal. We do so by applying high-order $\left(30^{\text {th }}\right.$-order $)$ polynomials, mimicking a Taylor-series approximation, to model the acausal error signal in the velocity trace. We are, therefore, claiming that there should be little or no motion shorter in period than our identified threshold period in the resulting velocity baseline, in line with our noise analysis in Figure $8 b$ above, and similarly attempting to produce a polynomial with nearly all its energy at periods longer than this same threshold. In the case of PS10, we conveniently have an error signal that is relatively large and temporally relatively clear of the large pulse of $S$-waves that contains the primary signal of interest. That is, the 
two signals are well-separated in time, frequency, and amplitude. Failed attempts to apply this method to causal analyses of the other two sites suggest that all three conditions may be important to the success of this technique, high-order polynomials not always behaving genially.

The fits to the complete causal velocity signals for PS10 (error plus signal) after deconvolution and demeaning are shown in Figure 11 while the fitted error functions themselves and their DFT amplitude spectra are shown in Figures $12 a, b$.

While there are precedents for using low-order polynomials in processing acceleration records (e.g., Graizer, 1979; Ellsworth et al., 2004), the questions naturally arises whether polynomials are appropriate here, whether such high-order polynomials are required, and whether the fitted polynomials are following the error portion of the traces faithfully or instead oscillating in some manner that distorts the signals of interest.

We note that there are Taylor-series approximations for sines, cosines, and the exponential - all the components of Fourier analysis. It can be argued, therefore, that polynomials are alternative basis functions for the decomposition of sinusoidal signals. We further note that our numerical experiments in fitting a real exponential at time and amplitude scales like those here demonstrated a need for high-order Taylor series ( $51^{\text {st }}$-order in that instance) for accurate fitting of such functions. These points suggested that high-order polynomials are candidates for modeling long-period sinusoidal features.

Following the example of Ellsworth et al. (2004) we tested cubic and other low-order polynomial fits to these velocity traces for removing the acausal features shown in Figures $11 a-c$ (the large $S$-wave/surface-wave packet is excluded from the fitting procedure, with this gap interpolated by the fitted polynomial). We had only partial success, with large residuals to the fits and obvious mismatches to the velocity trace - the curvature of the error signal is simply too great and clearly requires a "shorter period" fitting function. We systematically increased the polynomial order until at $19^{\text {th }}$-order the fit was reasonably close to the center of the trace before and after the large $S$-wave/surface-wave packet.

The resulting displacement traces at this point still showed some drift, very long-period displacement between the earliest and latest parts of the padded traces in a nearly linear manner. Increasing from a $19^{\text {th }}$ - to a $31^{\text {st }}$-order polynomial made little difference to the shorter-period por- 
tions of the displacement signal but effectively removed this long-period drift, so we adopted the higher order. Increasing beyond $31^{\text {st }}$ order seemed only to increase numerical noise.

To test the validity of the resulting baseline estimate, Figure $12 b$ shows that the frequency content of the polynomial is largely below the threshold period, as required, with a sharp cut-off between the energetic part of the band and the weak part. We infer that removing this polynomial effects a low-cut filter function of sorts, albeit a selective, model-fitting variant on that theme.

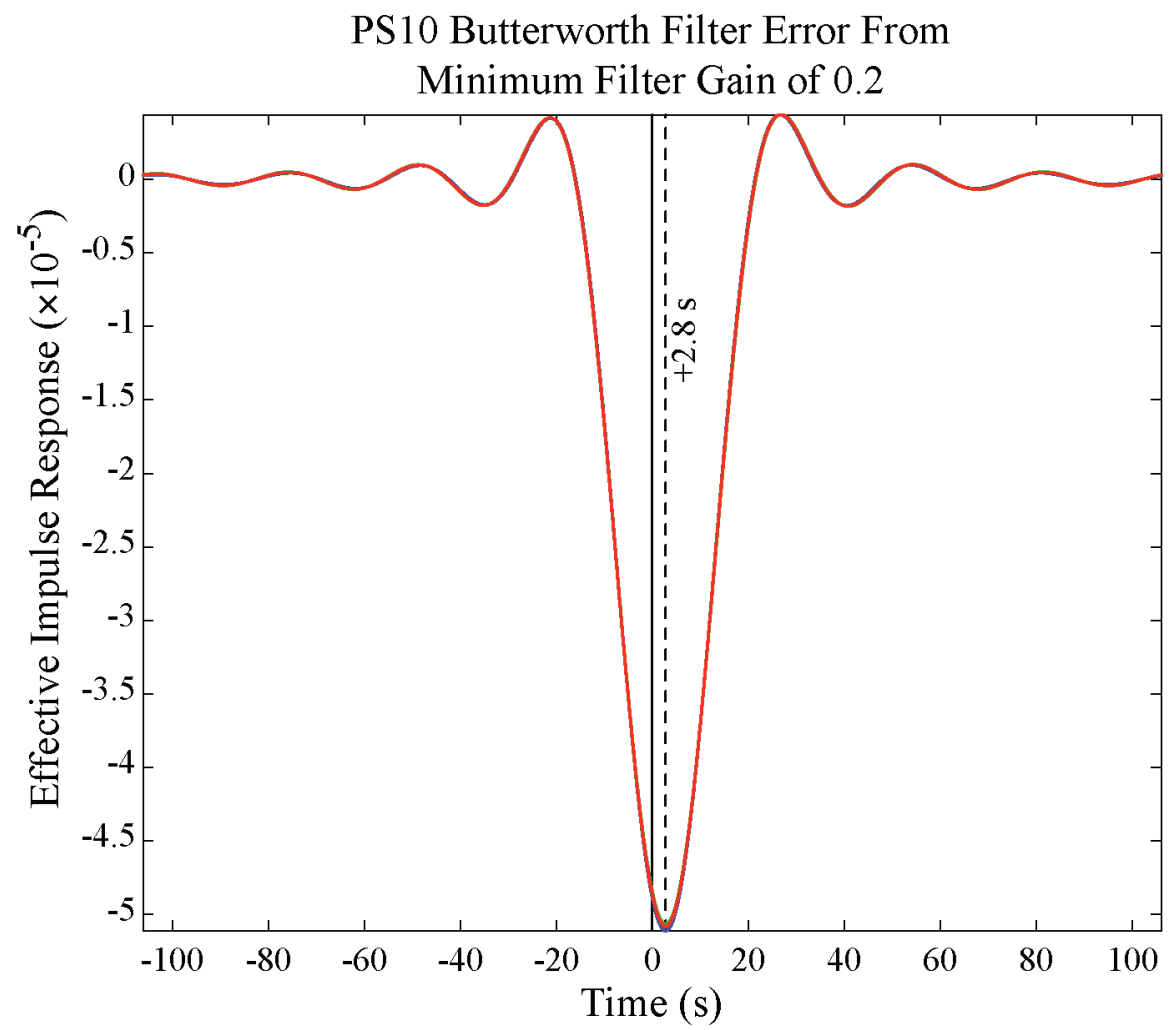

Figure 10. The difference between the impulse responses for the original Butterworth filter (the electronic filter used in the accelerograph) and the modified Butterworth filter used to deconvolve PS10 in "Option 2" processing. In effect, the acausal "error filter". 
(a) PS10 "Option 2" 30 th $_{\text {th }}$ order Polynomial Baseline Before Removal (L2 Fit);

Cosine Taper: Front $=5 \mathrm{~s}$; Rear $=2.3 \mathrm{~s}$

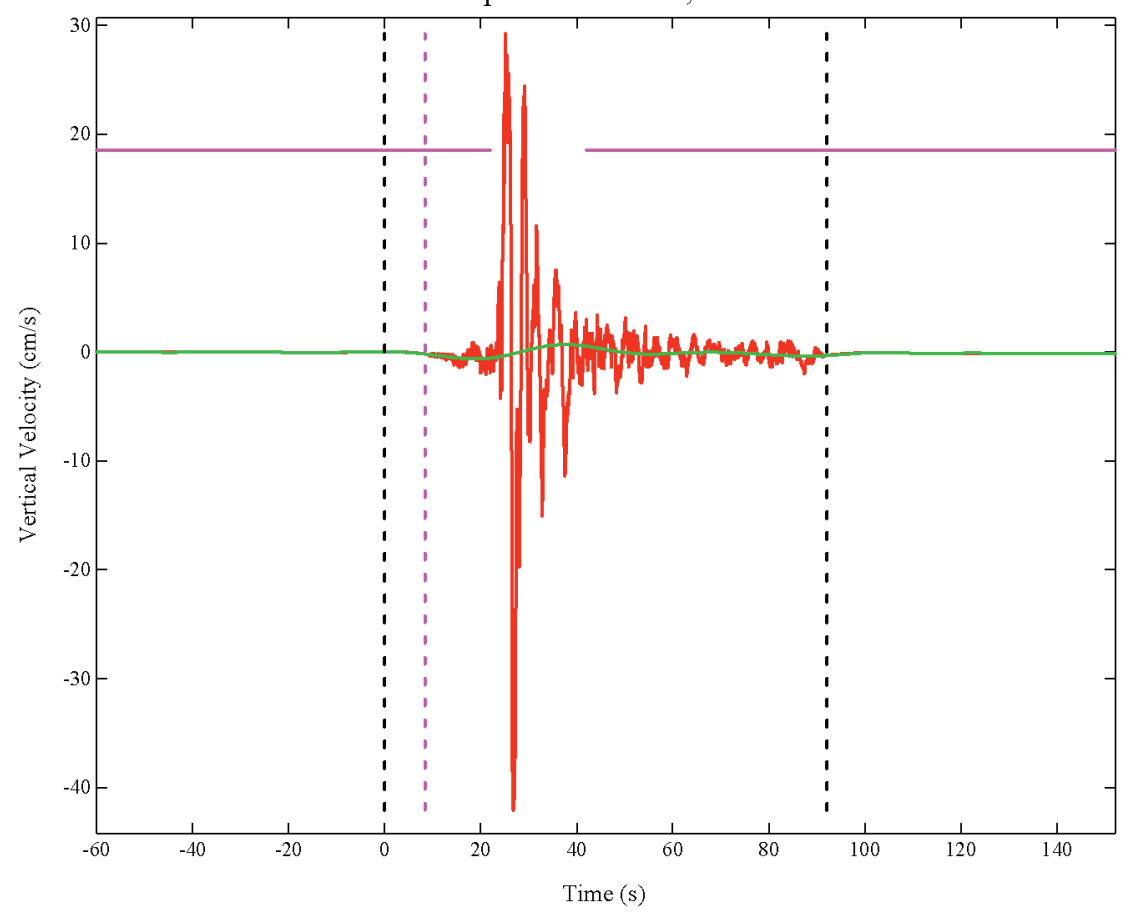

(b) PS10 "Option 2" 30 ${ }^{\text {th }}$-order Polynomial Baseline Before Removal (L2 Fit); Cosine Taper: Front $=5 \mathrm{~s}$; Rear $=5 \mathrm{~s}$

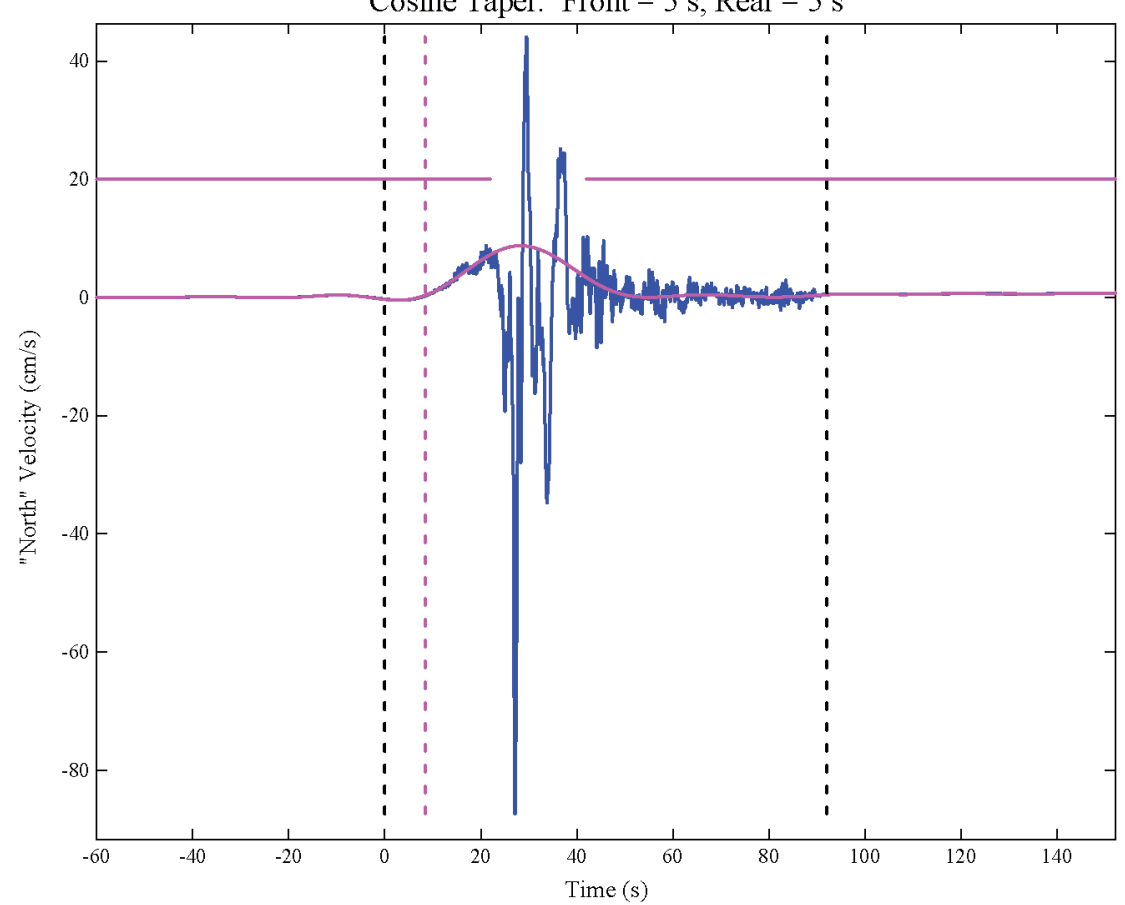

Figure 11. The velocity traces and $30^{\text {th }}$-order polynomial fits (contrasting color) for PS10 ("Option 2"). The pads are also plotted here and included in the fit. Vertical dotted lines show the extent of original seismograms, black, and the arrival time of the $P$-wave, magenta. The solid magenta line at the top of each plot shows the fitted portion of the traces - all but the strong part of the $S$-wave/surface-wave packet. (a) PS09; (b) PS10; and (c) PS11. 
(c) PS10 "Option 2" 30 th -order Polynomial Baseline Before Removal (L2 Fit);

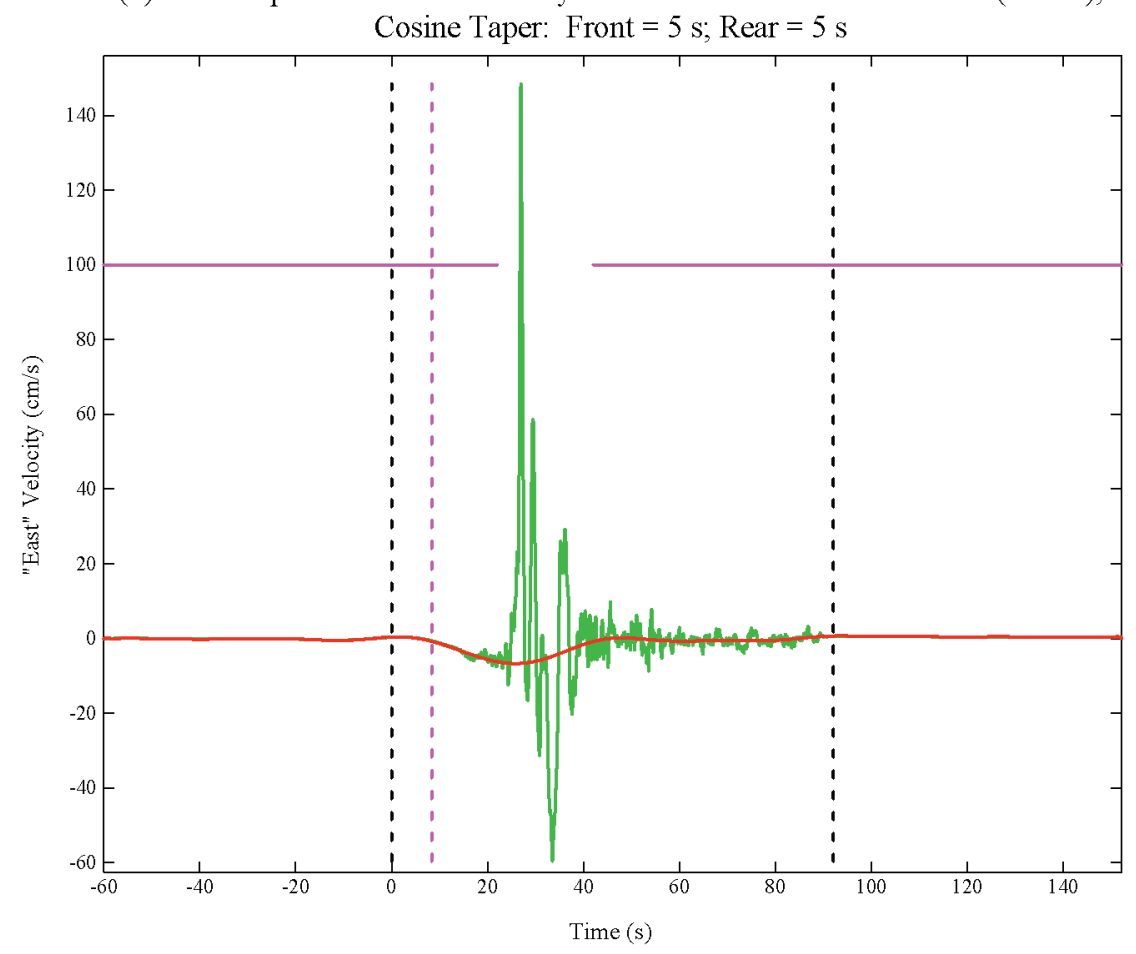

Figure 11. (cont.)

\section{Acausal Processing, All Stations}

For all other situations - PS09, PS10 "Option 1", and PS11 - the long-period noise in the signal is removed prior to deconvolution rather than being dealt with after deconvolution (Tables $6 a, b)$. With the noise thus suppressed at periods longer than the thresholds identified in Figures $8 a-c$, it is no longer necessary to raise the corresponding terms of the electronic Butterworth filter's DFT (the denominator of the deconvolution ratio) with the single exception of the $0-\mathrm{Hz}$ (DC) term which is always zero-valued. Raising this single frequency bin has a minimal effect on the Butterworth filter's impulse response - it creates a negligible "error filter" three orders of magnitude smaller than the one shown in Figure 10 and largely random in appearance.

To suppress the signal's long-period noise prior to deconvolution we use an acausal "Butterworth" filter, a $3^{\text {rd }}$-order Butterworth filter run both forward and backward across the accelerogram with corner periods as shown in Table 6, scaled from the threshold periods deduced in Figures $8 a-c$. This filtering technique is in keeping with the method of Boore (2005) and other authors, but here is closely tied to the needs of deconvolution. 


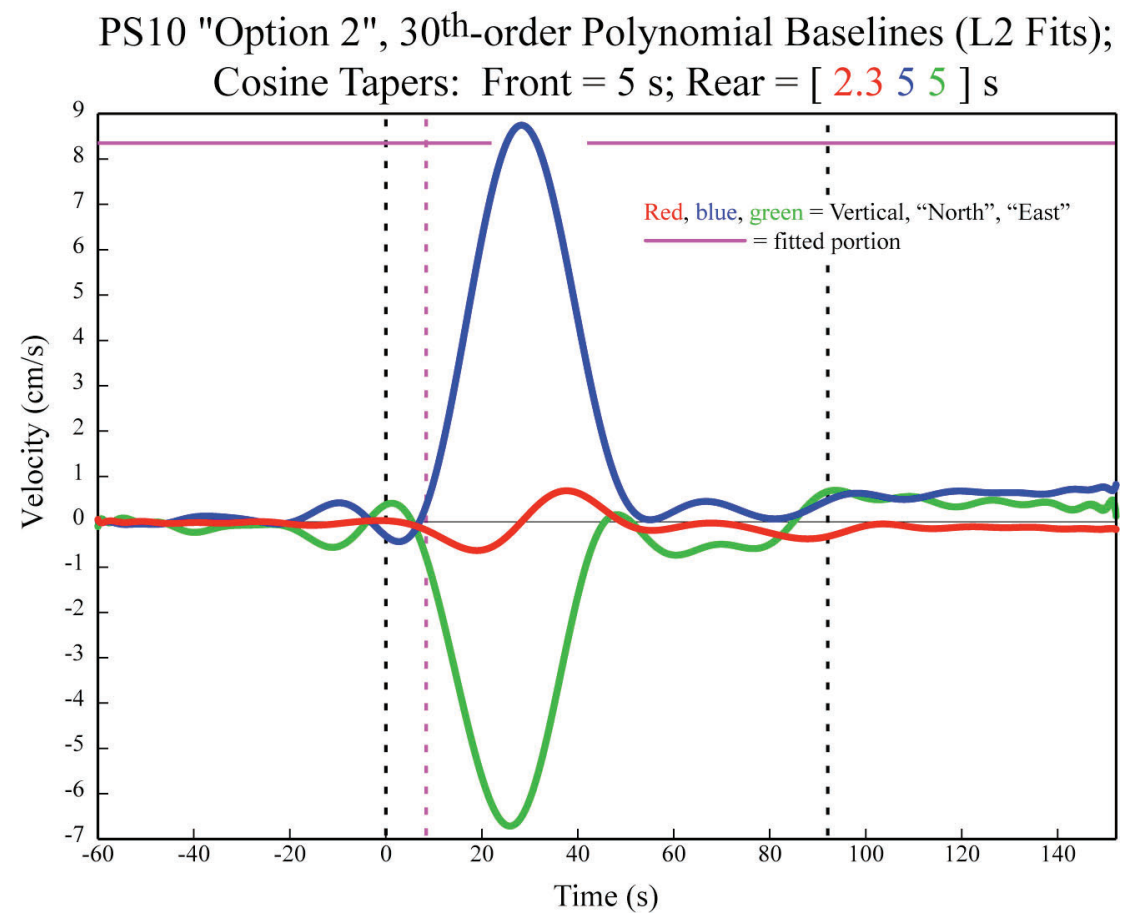

Figure 12a. From Figures $11 a-c$ the $30^{\text {th }}$-order polynomials fit by least squares to the velocity traces of PS10 ("Option 2") - in effect the modeled acausal error functions due to enforcing a minimum amplitude in the Butterworth filter deconvolution. Vertical dashed lines are at the limits of the original time series (black) and the arrival time of the $P$-wave (magenta). Horizontal magenta lines show constrained portions of traces.

\section{Integration}

The result of deconvolution is in all cases demeaned once more. Since the integration process is very sensitive to this initial value, we use the entire front-end pad and pre-event signal up to the $P$-wave arrival time to compute the value of this mean. (Attempts to use a shorter interval nearer the $P$-wave arrival time proved insufficient, producing large quadratic deviations in the displacement result.)

At last, the filtered or baseline corrected, deconvolved, demeaned acceleration trace is doubly integrated by the trapezoidal rule, beginning from the start of the front pad. As discussed by Boore (2005), including the pads in the integration is essential to obtaining a valid result. While we have done so, only the portions of this longer trace which correspond to the original acceleration trace are plotted in Figures 13a,b (PS09), Figures 14a,b (PS10, "Option 1"), Figures 15a,b (PS10, “Option 2”), and Figure 16 (PS11). 
For all sites the results are shown for the original components as oriented on the ground "North" is the local pipeline-parallel direction and "East" is $90^{\circ}$ clockwise from that azimuth. True azimuths are shown parenthetically for the unrotated components in Figures 13a, 14a, and $15 a$ and Figure 16 and enumerated in Tables $1 a-c$. In the case of PS09 and PS10 (Figures 13b, $14 b$, and $15 b$ ), results are also shown with the horizontal components rotated into the faultnormal $\left(21.62^{\circ}\right)$ and fault-parallel $\left(111.62^{\circ}\right)$ azimuths regionally prevalent near PS10 (Ellsworth et al., 2004).

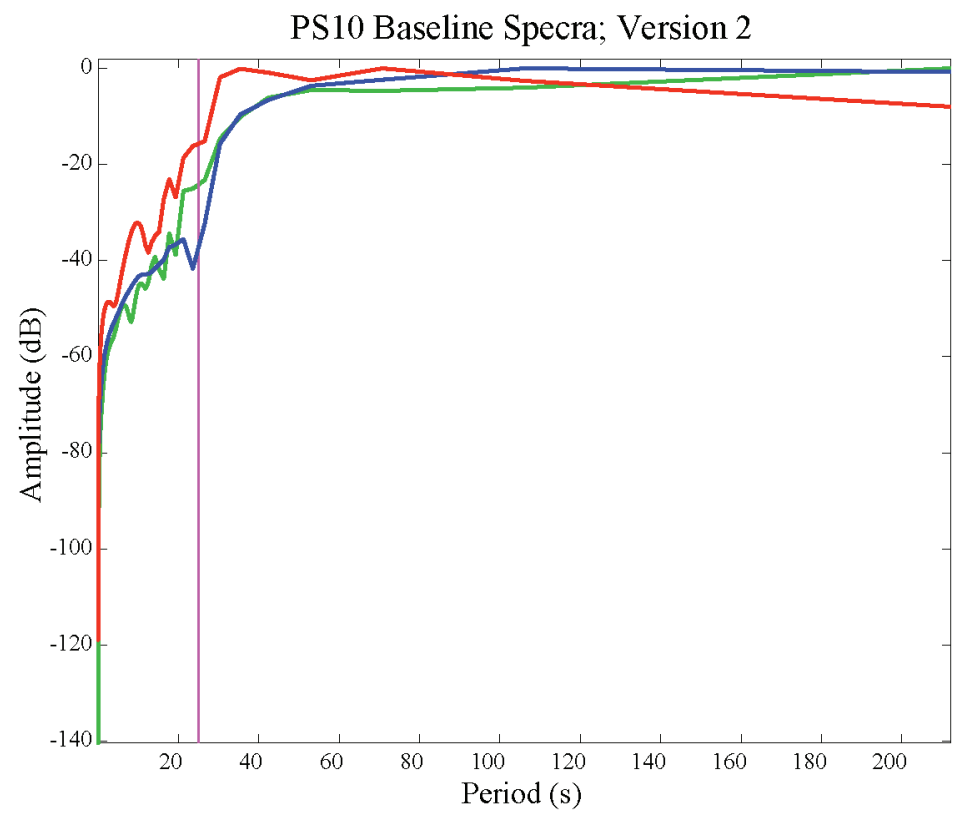

Figure 12b. DFT amplitude spectra of the polynomials in Figure $12 a$. The vertical magenta line is at the noise-threshold period determined from Figure $8 b$. Amplitudes are shown in decibels relative the largest amplitude in each spectrum. No window weighting is used, so effects of the boxcar window can be seen. 


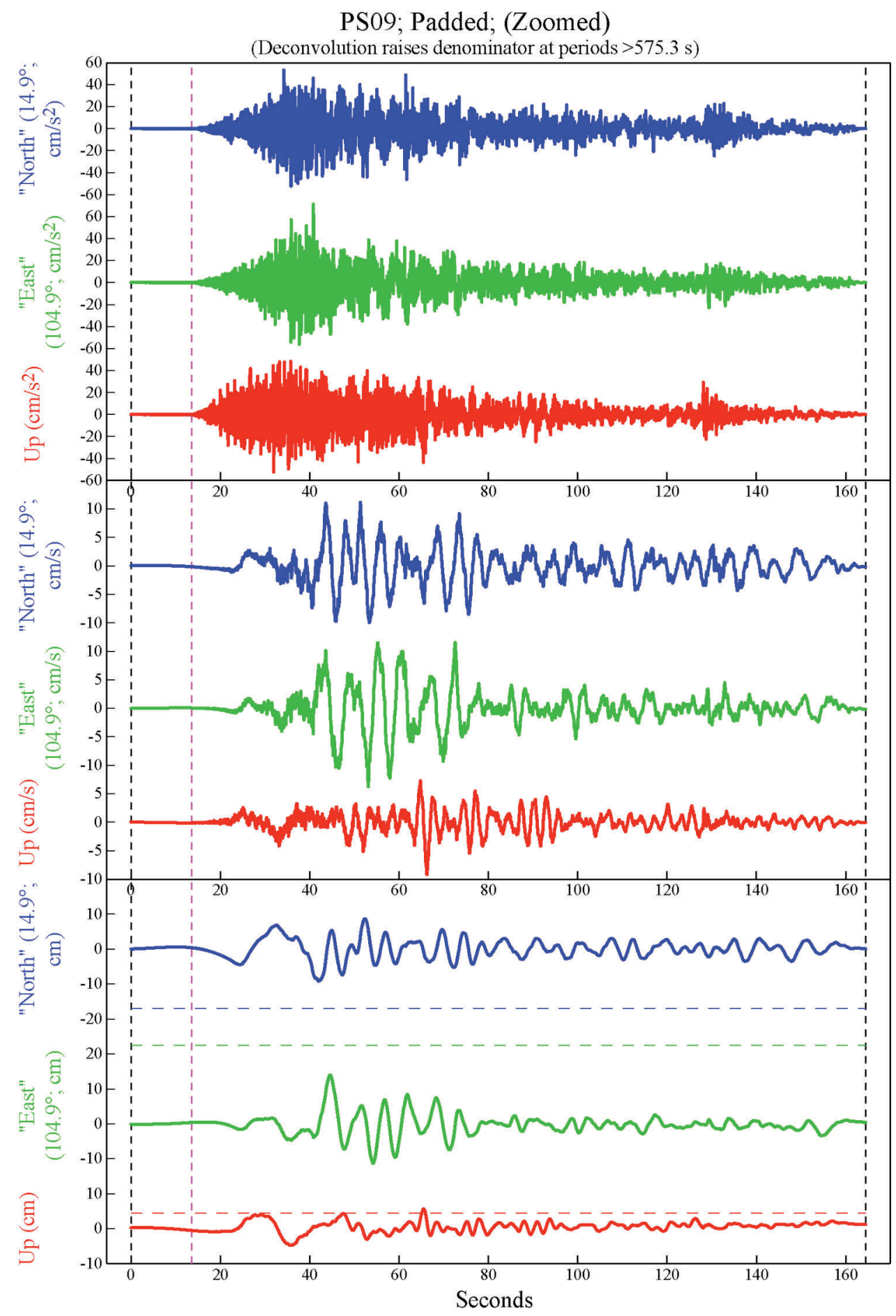

Figure 13a. Acceleration, velocity, and displacement for PS09 (processed as in Table 6a). Dashed horizontal lines in displacement are the geodetic model estimates of permanent ground displacement at this site (Hreinsdóttir et al., 2006), which we cannot model because of insufficiently resolved long-period signal. Sensor orientations are as emplaced, where "North" is local pipeline-parallel. Pads are included in all computations but only the time interval corresponding to the original acceleration trace is plotted (vertical black dashed lines). Vertical magenta dashed line is time of $P$-wave arrival. 


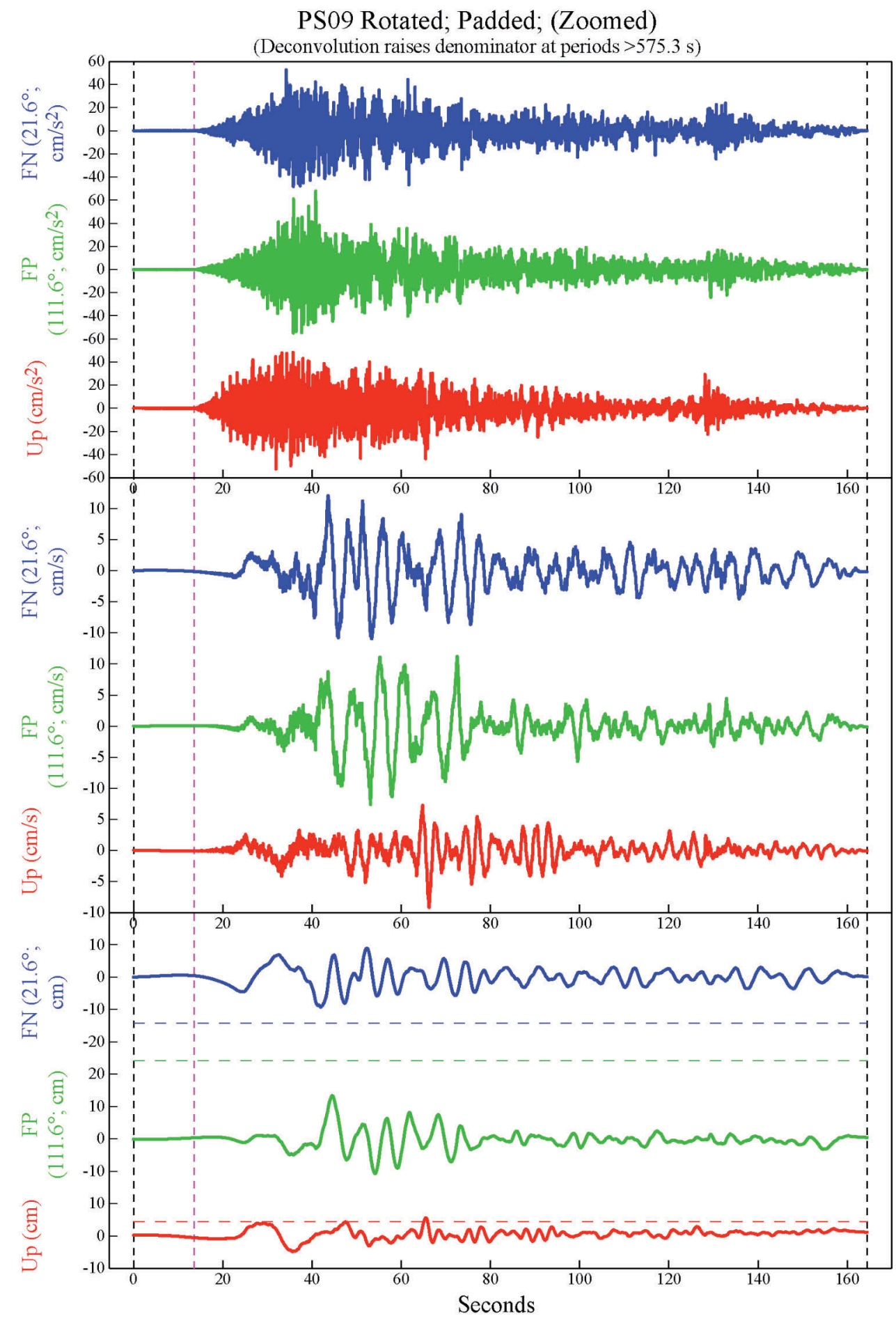

Figure 13b. The same as Figure $13 a$ but with the horizontal components rotated into the fault-normal (FN) and fault-parallel (FP) directions as they exist in the region around PS10. 


\section{Comments on Interpreted ("Volume 2") Seismograms}

The causal ("Option 2") analysis of PS10 (Figures 15a,b) and the results of Ellsworth et al. (2004) compare favorably, as do our "Option 2" results and the geodetic modeling of Hreinsdóttir et al. (2006). For the large, fault-parallel component of displacement (called "fling step" by Bolt and Abrahamson, 2003) our results are the lowest of the three, at $91 \%$ that of the geodetic model. The earlier Ellsworth et al. evaluation of PS10 seismic data is $123 \%$ of the geodetic estimate. Given that the geodetic value likely includes post-seismic relaxation, our estimate of the co-seismic displacement at PS10 may be the more accurate. However, it must be noted that the other two components of Figure $15 b$ mismatch the geodetic estimates by comparable or larger amounts, and these may simply be a measure of the combined errors of the two techniques, particularly including assumptions of the relevant fault azimuth.

The acausal results for PS10 are quite different in appearance (Figures 14a,b), all showing long-period premonitory oscillations before the $P$-wave arrival time (most noticeable in horizontal-component displacement traces). These features are characteristic responses of long-period acausal filters and no cause of concern but do point up the need to include the pads in integration.

By comparing Figures $14 a$ and Figures $15 a$ or of Figures $14 b$ and Figures $15 b$ it is apparent that the causal and acausal processing of PS10 has produced very similar acceleration and velocity records. Indeed, the short-period portions of the displacement traces are also very similar between these records. The critical difference is in the period range between about 15 and $30 \mathrm{~s}$. Shorter periods are similarly recovered between the causal and acausal cases but longer periods progressively differ, precisely as would be expected from the nature of the filters and polynomial error estimates applied to the processes. The polynomials appear to be successful at providing a filter-like removal of long-period noise signals without causing the ringing associated with highorder or brickwall filters.

It is clear from Figures $13 a, b$ and 16 that PS09 and PS11 do not approach the model GPS estimates of total ground displacement. As mentioned above, the resolution of the digitizers at these instruments appears to be insufficient to resolve the small long-period signals at these distal sites. Signal levels at long-periods (Figures 8a,c) drop into background noise levels at about 19 s, which from the analysis of PS10 appears to be the critical range for recovering these per- 
manent displacements. Nevertheless, from about $15 \mathrm{~s}$ upward in frequency, these are useful records for dynamic analysis.

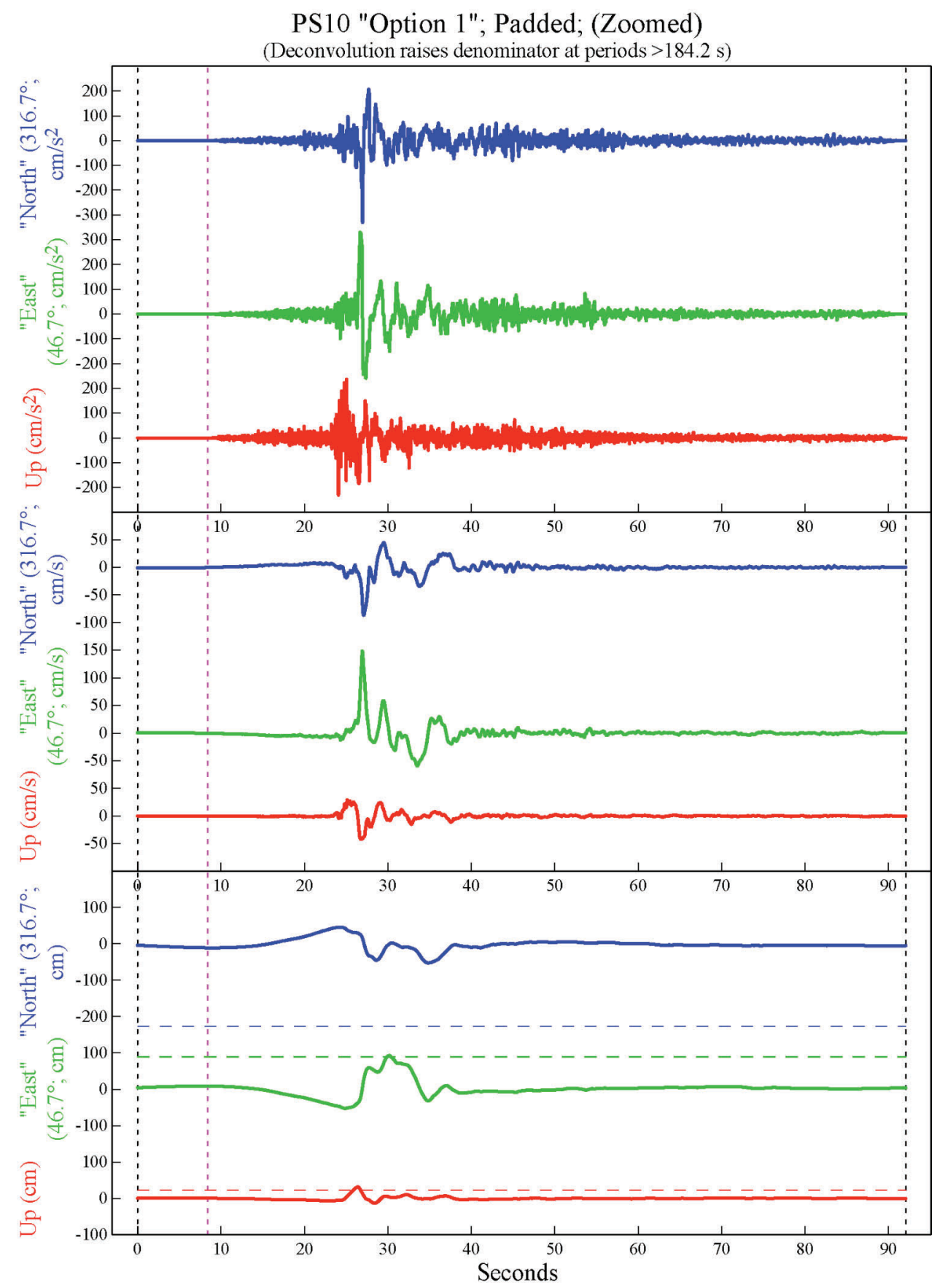

Figure 14a. Unrotated results for PS10, "Option 1" (processed as in Table 6b). Otherwise like Figure $13 a$. 


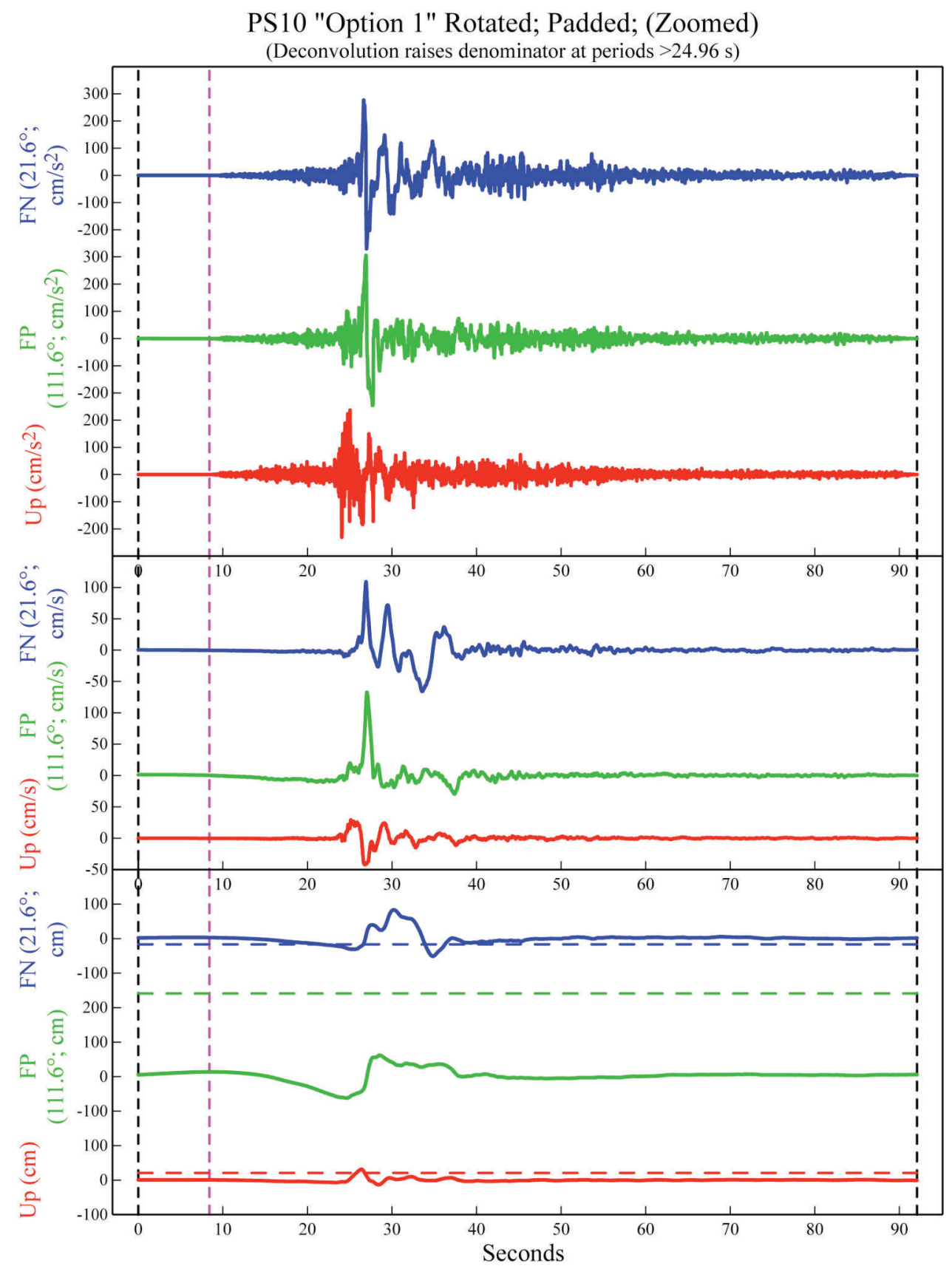

Figure 14b. Rotated results for PS10, "Option 1" (processed as in Table 6b). Otherwise like Figure $13 b$. 


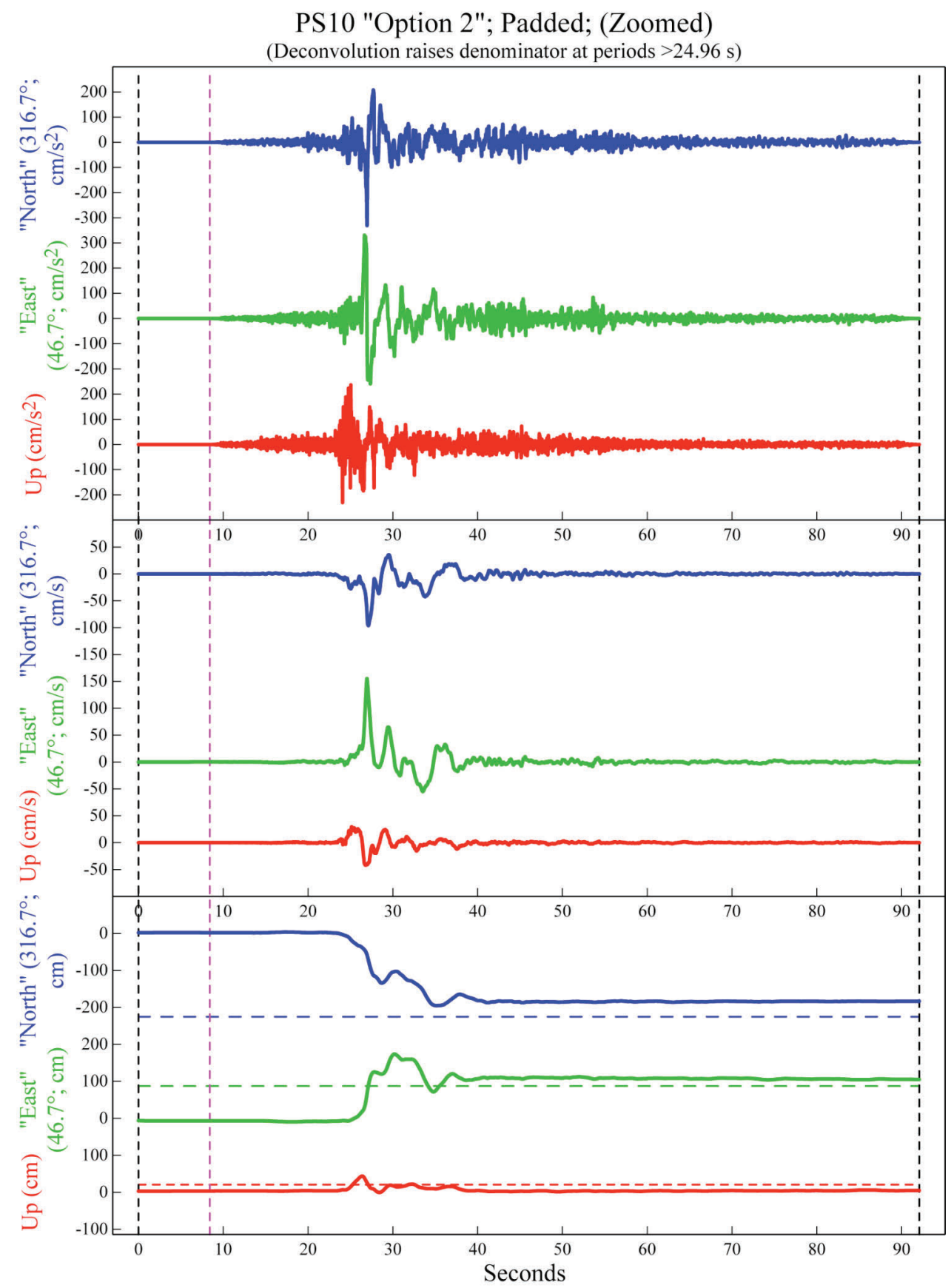

Figure 15a. Unrotated results for PS10, "Option 2" (processed as in Table 6b). Otherwise like Figure 13a. Shows near-field, "fling-slip" displacements, which compare to the dashed horizontal lines, the GPS modeling of Hreinsdóttir et al. (2006). 


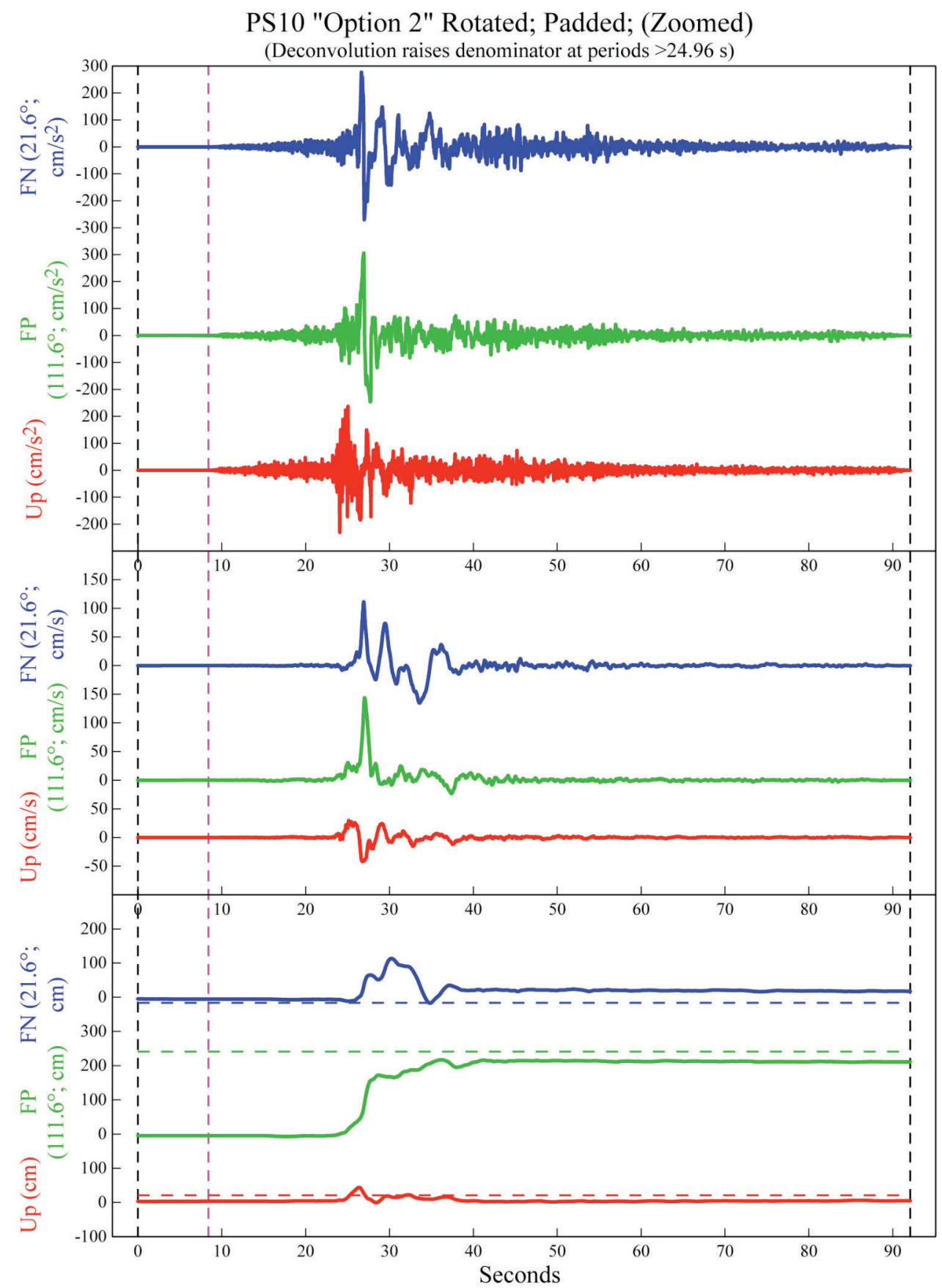

Figure 15b. Rotated results for PS10, "Option 2" (processed as in Table 6b). Otherwise like Figure $13 b$. Shows near-field, "fling-slip" displacements, which compare to the dashed horizontal lines, the GPS modeling of Hreinsdóttir et al. (2006). 
PS11; Padded; (Zoomed)

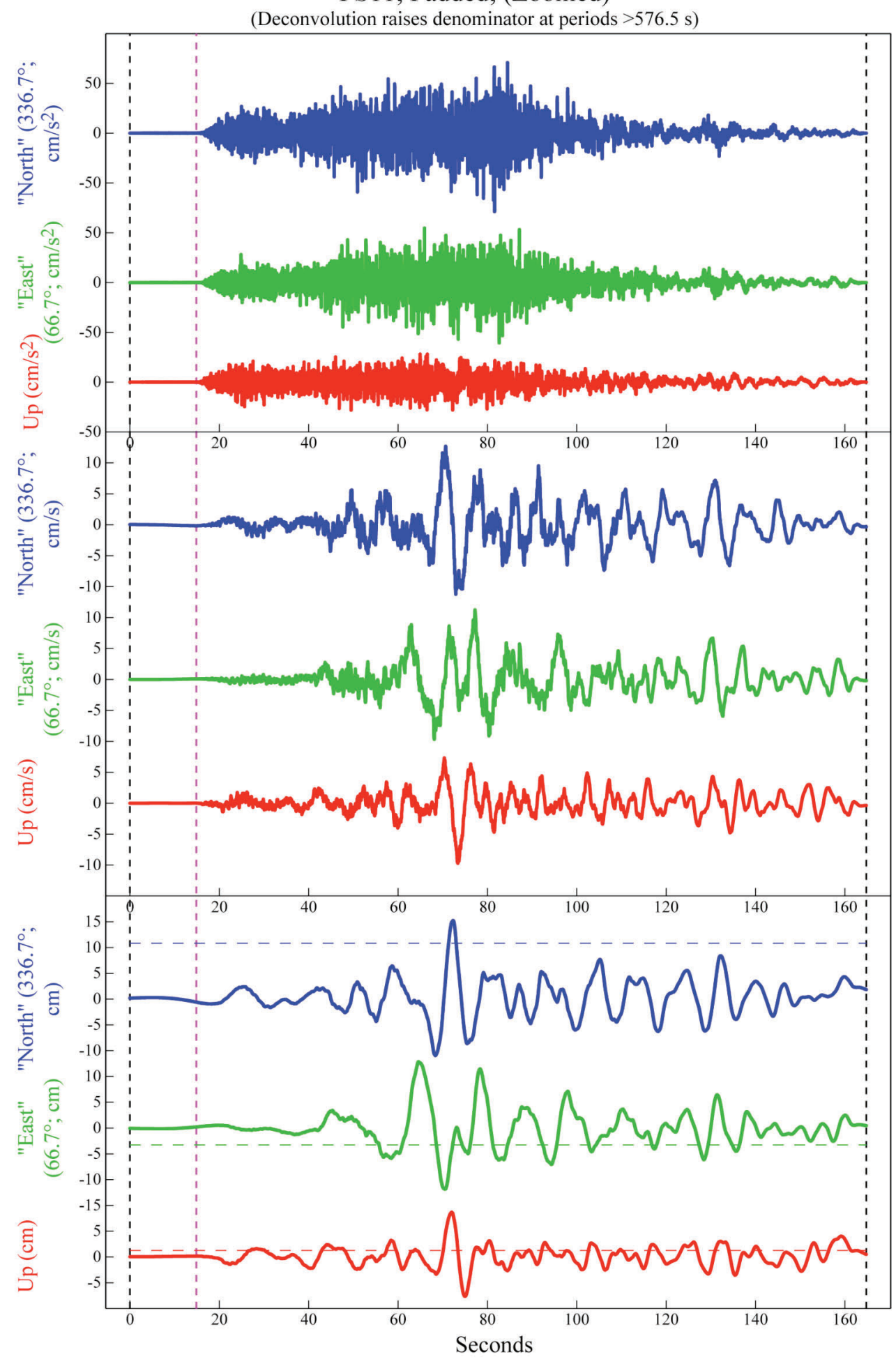

Figure 16. Unrotated results for PS11 (processed as in Table 6a). Otherwise like Figure 13a. 


\section{SEISMOGRAMS RELEASED WITH THIS REPORT}

The deconvolved accelerograms for all sites and their first and second integrals are the seismograms released here as "Volume 2" (interpreted) data. Because we are also releasing the acceleration records without these manipulations ("Volume 1", original accelerograms), there are two acceleration records for each component of each station, the processed and unprocessed records, and for PS10 yet another set of records for "Option 2" processing (Table 6b). The unprocessed, "Volume 1" records have only had our current set of gains and sensitivities removed but the instrument filter responses left in. None of the rotated traces are released with this report because they are easily computed from the unrotated traces.

All records are released in COSMOS Version 1.2 format because this format can preserve the full precision of the original record. USGS "SMC" format, and other "Blue Book" derived formats, although nominally sufficient for 16-bit data, have mantissas that are slightly too short to avoid an additional source of quantization noise within the range of these signals.

The seismograms released here can be considered the "final" versions, but a word of caution. We do not consider any particular processing scheme to be the only possible choice in the matter, as should be clear from the offering of two "Options" for PS10. There is a very real need in cases like PS10 to choose between causal and acausal processing in order to preserve one or another desired property of the records. Furthermore, there are matters of art that require varying degrees of subjectivity on the part of practitioners. In offering the "Volume 1" records and a detailed listing of the steps performed to arrive at the "Volume 2" records (Tables $6 a, b$ ), we hope to encourage others to try their hands at this set of records, or failing that at least to be aware of the abstruse art that created them.

All records are released in their originally recorded orientations, leaving it to the user to rotate them into their preferred orientations. When we rotated for presentation in Figures $13 b, 14 b$, and $15 b$, we did so after integration, believing that introducing another source of numerical noise prior to integration was unwise. The following are available at or by way of the Web site: 
- "Volume 1" acceleration records (gains and sensitivities only removed):

- PS09

- PS10

- PS11

- "Volume 2" interpreted acceleration, velocity, and displacement records:

- PS09

- PS10, "Option 1"

- PS10, "Option 2"

- PS11

- This report, 300 dpi TIFF versions of all figures, and Excel ${ }^{\mathrm{TM}}$ versions of all Tables.

- The original processing "diaries" detailing the steps followed and constants applied.

\section{ACKNOWLEDGEMENTS}

We wish to thank the many people of the Alyeska Pipeline Service Company, its contractors, and associates, as well as the hospitable people of Alaska for their great assistance and kindness during the challenging fieldwork and throughout this project. It is often people in the most challenging places who are the most reliable friends in times of need. We wish to thank David Boore for his unerring guidance and mentoring in the difficult effort of processing these unusual records, particularly in matters of baseline correction. We also thank Bob Hutt and Joe Fletcher for their characteristically erudite, incisive, and very helpful reviews of the manuscript.

\section{REFERENCES}

ANSS TIC, Technical Guidelines for the Implementation of the Advanced National Seismic System, U.S. Geol. Surv. Open File Rep., 02-92, 92 pp., 2002.

Boore, D. M., On pads and filters: processing strong-motion data, Bull. Seismol. Soc. Am., 95, no. 2, 745-750, 2005.

Boore, D. M., and S. Akkar, Effect of causal and acausal filters on elastic and inelastic response spectra, Earthquake Eng. Struct. Dyn., 32, 1729-1748, 2003.

Converse, A. M., and A. G. Brady, BAP: basic strong-motion accelerogram processing software; version 1.0, U.S. Geol. Surv. Open File Rep., 92-296A, 177 pp., 1992. 
Ellsworth, W. L., M. Çelebi, J. R. Evans, E. G. Jensen, R. Kayen, M. C. Metz, D. J. Nyman, J. W. Roddick, P. Spudich, and C. D. Stephens, Near-Field Ground Motion of the 2002, Denali Fault, Alaska, Earthquake Recorded at Pump Station 10, Earthquake Spectra, 20, no. 3, 597-615, 2004.

Graizer, V. M., Determination of the true ground displacement by using strong motion records, Izvestiya, Phys. Solid Earth, 15, no. 12, 875-885, 1979.

Hreinsdóttir, S., J. T. Freymueller, R. Bürgmann, and J. Mitchell, Coseismic deformation of the 2002 Denali Fault Earthquake: Insights from GPS measurements, J. Geophys. Res., doi:10.1029/2005JB003676, (in press), 2006.

Hreinsdóttir, S. , J. T. Freymueller, R. Bürgmann, and J. Mitchell, Coseismic deformation of the 2002 Denali Fault earthquake: Insights from GPS measurements, J. Geophys. Res., 111, B03308, 18 pp., doi:10.1029/2005JB003676, 2006.

Kayen, R., E. Thompson, D. Minasian, R. E. S. Moss, B. D. Collins, N. Sitar, D. Dreger, and G. Carver, Geotechnical reconnaissance of the 2002 Denali Fault, Alaska, earthquake, Earthquake Spectra, 20, no. 3, 639-667, 2004.

Larson, K. M., P. Bodin, and J. Gomberg, Using 1-Hz GPS data to measure deformations caused by the Denali fault earthquake, Science, 300, 1421-1424, 2003.

Wielandt, E., Seismic sensors and their calibration, in: New Manual of Seismological Observatory Practice (NMSOP), Chapter 5, ed. P. Bormann, IASPEI and GeoForschunsgZentrum, Potsdam, 5.1-5.45, 2002. 